\title{
Abstracts \\ of the 2016 Biennial Meeting of the American Society for Stereotactic and Functional Neurosurgery Chicago, IL, USA, June 18-21, 2016
}

\author{
Guest Editors: \\ Konstantin V. Slavin, Chicago, IL \\ Brian H. Kopell, New York, NY \\ Peter E. Konrad, Nashville, TN \\ Joshua M. Rosenow, Chicago, IL
}

\section{ORAL PRESENTATIONS}

\section{Subthalamic Local Field Potentials in Parkinson's Disease and Isolated Dystonia: An Evaluation of Potential Biomarkers}

Doris D. Wang, Coralie de Hemptinne, Svjetlana Miocinovic, Jill L. Ostrem, Philip A. Starr

\section{Introduction}

Local field potentials (LFP) recorded from the subthalamic nucleus in patients with Parkinson's disease (PD) demonstrate prominent oscillations in the beta $(13-30 \mathrm{~Hz})$ frequency range, and reduction of beta band spectral power by levodopa and deep brain stimulation (DBS) correlates with motor symptom improvement. Several features of beta activity have been theorized to be specific biomarkers of the parkinsonian state, though these have rarely been studied in non-parkinsonian conditions.

\section{Methods}

To compare resting state LFP features in PD and isolated dystonia and evaluate disease-specific biomarkers, we recorded subthalamic LFPs from 28 akinetic-rigid PD and 12 isolated dystonia patients during awake DBS implantation. Spectral power and phase-amplitude coupling characteristics were analyzed.

\section{Results}

In 26/28 PD and 11/12 isolated dystonia patients, the LFP power spectrum had a peak in the beta frequency range, with similar amplitudes between groups. Resting state power did not differ between groups in the theta $(5-8 \mathrm{~Hz})$, alpha (8-12 $\mathrm{Hz})$, beta $(13-30 \mathrm{~Hz})$, broadband gamma $(50-200 \mathrm{~Hz})$, or high frequency oscillation (HFO, 250-350 Hz) bands. Analysis of phase-amplitude coupling between low frequency phase and HFO amplitude revealed significant interactions in 19/28 PD and 6/12 dystonia recordings without significant differences in maximal coupling or preferred phase.

\section{Conclusion}

Two features of subthalamic LFPs that have been proposed as specific parkinsonian biomarkers, beta power and coupling of beta phase to HFO amplitude, were also present in isolated dystonia, including focal dystonias. This casts doubt on the utility of these metrics as disease-specific diagnostic Biomarkers.

\section{Stereotactic Accuracy of a Compact, Mobile Intraoperative MRI}

Nina Kohn, Dishen Lin, Daniel J. Markowitz, Sussan Salas, Michael Schulder

Introduction

Intraoperative imagers provide neurosurgeons with real-time information required to maintain precise navigation during surgery. In this study, we assessed the stereotactic accuracy of a compact, intraoperative magnetic resonance imager (iMRI), the 0.15 Tesla (T) PoleStar N30 (PN30).

Methods

Images were acquired using a water-filled phantom model of the brain. The phantom was scanned using T1-weighted, T2weighted, PSIF, and FLAIR sequences. Data collected with the PN30 were compared with those obtained in a previous study assessing the PoleStar N20 (PN20), an earlier model of this iMRI system. Additionally, the stereotactic accuracy of PN30 was measured against that of standard surgical navigation on a $1.5 \mathrm{~T}$ diagnostic scan MRI using T1 weighted images (with the same water phantom).

$\underline{\text { Results }}$

Navigation with PN30 images was more accurate than that using diagnostic MRI. Mean error with the PN30 using T1W images was $1.24 \pm 0.47 \mathrm{~mm}$ and $1.28 \pm 0.49 \mathrm{~mm}$ with T2W images, vs $2.43 \pm 0.81 \mathrm{~mm}$ for navigation based on T1W images from the $1.5 \mathrm{~T}$ scan $(95 \% \mathrm{CI}, \mathrm{p}=0.016$ and 0.001 , respectively). This higher degree of accuracy with iMRI-based navigation may reflect the ability to bypass the registration that is needed when employing a scan acquired before surgery, a step that introduces another source of error into the process. Conclusion

A high degree of stereotactic accuracy can be achieved with a compact, low field iMRI. Improvements in magnet design can yield progressive increases in accuracy, validating the concept of these devices designed for use during intracranial surgery. Avoiding the need for registration between image and surgical space also can increase navigation accuracy. 


\section{Atlas-Independent, Individualized Mapping of the Optimal Locus of Subthalamic DBS}

\section{Erin Conrad, Kelvin Chou, Parag G. Patil}

Introduction

The degree of symptomatic improvement among Parkinson's disease (PD) patients undergoing subthalamic deep brain stimulation (STN DBS) is highly variable. One reason for this variability may be differences in the location of active electrode contacts relative to some currently undefined optimal locus of stimulation. Our objective was to determine this ideal location, based entirely on individual patient anatomy and independently of any atlas-based generalizations. Methods

We used a validated 3T MRI protocol and high-resolution CT imaging to precisely determine the location of individual DBS active contacts relative each patient STN. In 20 patients treated with STN DBS for Parkinson's disease, we developed a computational electrical field model of neuronal activation weighted by Unified Parkinson's Disease Rating Scale improvement. Our novel method allowed unconstrained identification of the locus of ideal stimulation across patients. $\underline{\text { Results }}$

Based on both outcomes analysis and our electrophysiological model, the average location of the leads associated with greatest symptom improvement at the lowest voltage was in a region dorsal, just posterior, and just lateral to the anatomical midpoint of the subthalamic nucleus. Interestingly, more anterior leads were associated with significantly better outcomes than more posterior leads.

Conclusion

Our results suggest that the optimal locus of stimulation in DBS for Parkinson's disease is at the dorsal border just above the STN. They also suggest that it may be beneficial to place leads further anterior than is current practice.
Atlas-Independent, Individualized Thalamic Segmentation and Tissue Activation Modeling of Deep Brain Stimulation for Essential Tremor

Layla Houshmand, Ashutosh Chaturvedi, Kelvin Chou, Cameron McIntyre, Parag G. Patil

Introduction

The mechanisms underlying DBS-mediated control of essential tremor (ET) remain unknown. To date, computational models of DBS stimulation have relied on atlas-based definitions of thalamic nuclei. However, recent advances in diffusion-tensor MRI and computational modeling have raised the possibility of an atlas-independent, strictly individualized approach. In this study, we compare this novel approach to conventional methods to understand both DBS effects on tremor and its most common side effects (sustained paresthesias and dysarthria).

Methods

For 10 patients treated with unilateral, left thalamic DBS for ET, we compared the results of atlas-based and atlasindependent (A-I) thalamic segmentation and voltage threshold of activation (VTA) modeling. For A-I segmentation, we utilized a novel $\mathrm{k}$-means clustering algorithm to delineate thalamic nuclei from each patient's individual DTI images. A-I VTA modeling was then performed from individual patient DTI-based conductivity tensor fields. We compared VTA overlap volumes with anatomic regions of interest and correlated them to tremor control, sustained paresthesias, and dysarthria.

Results

A-I thalamic segmentation compared well with atlas-based results. The centroid locations of 9 of 13 thalamic nuclei were statistically invariant between A-I and atlas-based segmentations. All 10 of 10 A-I VTAs for the active DBS contact at the clinically determined stimulation settings were localized, as expected, to the motor thalamus. By comparison, only 9 of 10 atlas-based VTAs overlapped with the motor thalamus for the same stimulation settings. Individualized VTA predicted dysarthria resulting from internal capsule (IC) stimulation $(\mathrm{p}<0.05)$ whereas atlas-based VTA modeling does not $(p=0.71)$. There was no statistically significant difference in sensory thalamus stimulation between models during sustained sensory paresthesias (paired t-test, $\mathrm{p}=0.25$ ). Conclusion

While traditional atlas-based thalamic segmentation and VTA modeling techniques provide reliable means of DBS targeting and mechanistic study, improved precision may be possible with an approach based on fully individualized DTI imaging data. 
Incisionless Transcranial MR-guided Focused Ultrasound in Essential Tremor: Cerebellothalamic Tractotomy

Marc N. Gallay, Daniel Jeanmonod

$\underline{\text { Introduction }}$

Targeting of the subthalamus was explored in the past by different functional neurosurgical groups applying the radiofrequency technique to treat patients suffering from essential tremor (ET). Recent advances in magnetic resonance guided focused ultrasound technology (MRgFUS) offer the possibility to perform thermocoagulation of the cerebellothalamic fiber tract in the subthalamus without brain penetration, allowing a strong reduction of the procedurerelated risks and increased accuracy. We present here the first results of the MRgFUS cerebellothalamic tractotomy (CTT). Methods

21 consecutive patients suffering from chronic, therapyresistant ET were treated with an MRgFUS CTT. 3 patients received bilateral treatment with 1 year interval. Primary relief assessment indicators were the Essential Tremor Rating Scale (ETRS) taken at follow up (3 months to 2 years) with accent on the Hand Function subscores (HF16 for treated hand and HF32 for both hands) and handwriting. Patients with HF32 above 28 points over 32 (Group 1) were analysed separately from the rest (Group 2).

$\underline{\text { Results }}$

Mean ETRS score for all patients was 57.6+/-13.2 at baseline, and 25.8+/-17.6 at 1 year $(\mathrm{n}=10)$. The HF16 score reduction was $92 \%$ in Group 2 at 3 months and stayed stable at 1 year (90\%). Group 1 showed only an improvement of $41 \%$ at 3 months and $40 \%$ at 1 year. Nevertheless, two patients of Group 1 treated bilaterally had an HF16 score reduction of $75 \%$ and $88 \%$ for the dominant hand at 1 year after second side.

\section{Conclusion}

CTT with MRgFUS was shown to be an effective and safe approach for patients with therapy-refractory essential tremor, combining neurological function sparing with precise targeting and the possibility to treat patients bilaterally.
MR Guided Focused Ultrasound Thalamotomy for the Treatment of Essential Tremor: Pivotal Study Results

Ryder Gwinn, Nir Lipsman, Travis S. Tierney, Howard M. Eisenberg, Pejman Ghanouni, W. Jeffrey Elias

Introduction

A recent study showed technical feasibility to improve symptoms in patients with essential tremor utilizing MR guided focused ultrasound to create a lesion in the thalamus. A randomized, controlled, double-blinded trial was therefore conducted to demonstrate safety and efficacy of this treatment. Methods

Seventy-six patients with severe medically refractory essential tremor were randomized to receive either unilateral MR guided focused ultrasound thalamotomy or sham lesion (3:1) after meeting inclusion/exclusion criteria for study participation. Primary outcome measure was upper limb tremor score on a subset of the Clinical Rating Scale for Tremor (CRST), for the arm contralateral to thalamotomy 3 months post treatment. Secondary measures included safety, treated arm tremor score at one year, total tremor score at 3 and 12 months, quality of life, and disability scores. Outcome measurements were obtained at $1,3,6$, and 12 months following treatment.

$\underline{\text { Results }}$

Mean contralateral hand tremor improved by 47\% (18.1 to 9.6 on CRST subscores, $\mathrm{p}<0.001$ ) compared to baseline, and remained significantly improved at 12 months $(10.6, \mathrm{p}<0.001)$. Sham treatment arm and ipsilateral limb to treatment showed no statistical improvement at 3 months. Quality of life scores improved by $54 \%$ in the treatment group compared to $1 \%$ in the sham at 3 months. Total disability at 3 months statistically improved only in the treatment group ( 16.5 to $6.2, \mathrm{p}<0.001)$, and remained improved at 12 months $(6.3, \mathrm{p}<0.001)$. One serious treatment related adverse event (treated hand hypesthesia) was observed in the 12 months following treatment.

Conclusion

MR guided focused ultrasound thalamotomy can be performed in awake patients with a high degree of safety, and produces significant, durable improvements in contralateral hand tremor scores, disability, and quality of life. 
Rates of Serious Adverse Psychiatric Events PostSubgenual Cingulate Cortex Deep Brain Stimulation for Psychiatric Illness: A Single-Centre, Prospective FollowUp Study

Peter Giacobbe, Andres M. Lozano, Nir Lipsman, Francesco Sammartino, Nathan Christopher Rowland

Introduction

It is unknown whether Subgenual Cingulate Cortex (SCC)

Deep Brain Stimulation (DBS), an investigational neurosurgical intervention for patients with treatment-resistant psychiatric illness, is associated with decreases in serious adverse psychiatric events, such as suicide attempts or hospitalizations.

Methods

A prospective follow-up study of all patients who received SCC DBS for a psychiatric indication $(n=81)$ at the University Health Network in Toronto from May 2003 to the present. The long-term follow-up (LTFU) time was calculated for each patient, defined as the time in months from surgery to the time of dropout for whatever reason, including removal of DBS, withdrawal of consent or death. A within-subjects analysis was performed comparing rates of suicide attempts and psychiatric hospitalizations for each patient during LTFU and the equivalent pre-DBS period.

$\underline{\text { Results }}$

A total of 4402 patient-months (366.8 patient-years) of followup were provided to all groups. Patients receiving SCC DBS for depression represented the bulk of the LTFU (3658 patient-months or 304.8 patient-years), followed by Anorexia Nervosa (650 patient-months or 54.2 patient-years) and Bipolar Disorder (94 patient-months or 7.8 patient-years). Prospectively across all diagnostic groups, 16 suicide attempts were made post-SCC DBS, compared to 40 attempts during the comparable pre-DBS LTFU period (pairwise t-test $=2.13$, $\mathrm{p}<0.005)$. There were 4 completed suicides. Rates of hospitalization were markedly decreased in the post-DBS LTFU compared to the identical pre-DBS period (23 vs. 100 hospitalizations) (pairwise t-test $=3.58, \mathrm{p}<0.001$ ). Conclusion

This represents the largest single-centre experience of SCC DBS for psychiatric illness in the world. In a group of patients with extreme levels of treatment-resistance to conventional treatments, using a within-subject analysis, DBS was associated with a decrease in the rate of suicide attempts and hospitalizations post-surgery. The estimated frequency of completed suicide was 1 per every 1100 patient-months of follow-up.

\section{Long-Term Outcome of Dorsal Anterior Cingulotomy for Treatment-Resistant Depression}

Danika L. Paulo, Paul McCormick Jr., Anna Gilmour, Rachel Franklin, Valerie Giorgione, Sameer A. Sheth, Emad N. Eskandar, Darin D. Dougherty

Introduction

Approximately $10-30 \%$ of patients with depression are refractory to conventional treatments, including pharmaceuticals, behavioral therapy, and electroconvulsive therapy. Dorsal anterior cingulotomy has been used for treatment-resistant depression (TRD) since its development in the 1960s, but few long-term studies exist.

Methods

We performed a retrospective review of 45 patients who underwent cingulotomy for TRD at the Massachusetts General Hospital between 1991 and 2014. Of these patients, 25 had pre- and postoperative Beck Depression Inventory (BDI) scores. Reduction in BDI by $>=50 \%$ was considered a full response. We calculated responder rate and mean change in BDI at initial and last follow-up.

$\underline{\text { Results }}$

At initial follow-up (mean 21 months), responder rate was $40 \%$, with a mean BDI decrease of $33 \%$. At last follow-up (mean 40 months), responder rate was $52 \%$, with a mean BDI decrease of $52 \%$. Broken into quartiles, average BDI change was $-94 \%,-73 \%,-36 \%$, and $-9 \%$.

Conclusion

We provide long-term outcome data from a large series of patients undergoing cingulotomy for TRD. The response rate of $52 \%$ in this series is comparable to that of previous studies, even with longer follow-up. The distribution of change in BDI across quartiles demonstrates the overall benefit obtained within this cohort. Cingulotomy remains an effective option for patients with TRD. 


\section{The Proper Target for OCD DBS is Individualized for Each Patient Along the Striatum Depending on the Content of the Obsessions}

Juan A. Barcia, Josue Moises Avecillas-Chasin, Jose Angel Pineda-Pardo, Rocío Arza, Blanca Reneses, Julia GarciaAlbea, Bryan Strange

Introduction

Although different targets are used to treat obsessivecompulsive disorder (OCD) with deep brain stimulation (DBS), the overall results (about $50 \%$ of responders) have not improved from the lesion-making era. Probably this is because OCD is a heterogeneous disease, with different symptomatic dimensions. We hypothesized that the optimal target is individualized for each patient, related to the symptomatic dimension. Further, we tested the possibility to use functional and structural connectivity to predict it.

\section{Methods}

We conducted a prospective, randomized, double blinded study in seven OCD patients. A fMRI Maudsley's test showing pictures related to several symptomatic OCD contents was performed. Then, the striatum was segmented using the projections of ventromedial, orbitofrontal, dorsolateral and anterior cingulate cortices. A tetrapolar electrode (Medtronic) was inserted along the striatum using a trajectory in which each contact was closest to each segment. Patients were stimulated using a random series of five periods (four contacts plus a zero volts activation) during three months, separated by one month washout period. Patients were evaluated for clinical and neuropsychological effects by an observer blind to the active contact

\section{$\underline{\text { Results }}$}

Six patients $(85.71 \%)$ were responders to any contact, with a mean YBOCS reduction of $50,84 \%$, while only three $(42.86 \%)$ responded to the most distal contact, (mean YBOCS reduction: $21.69 \%$ ). Patients showing obsessions and compulsions related to body danger responded best to the more ventral contacts (two cases), while those showing symptoms with ideatory contents or those related to checking (or ordering) responded best to more dorsal contacts. We found a relationship between the volume of tissue activation in each contact and the tracts crossing from the cortical activated area after the Maudsley's test towards the striatum.

Conclusion

These results suggest that there is an individualized proper DBS target depending on the contents of obsessions in OCD patients.

\section{Deep Brain Stimulation of the Basolateral Nucleus of the Amygdala for Treatment-Refractory Post-Traumatic Stress Disorder}

Jean-Philippe Langevin, Ralph Koek, Holly Schwartz, James Chen, David Sultzer, Mark Mandelkern, Alexis Kulick, Scott Krahl

Introduction

Amygdala hyperactivity plays a crucial role in post-traumatic stress disorder (PTSD) by mediating failure of fear extinction. Deep brain stimulation (DBS) can modulate the functional output of the amygdala to permit fear extinction. We have previously shown that DBS of the basolateral nucleus of the amygdala (BLn) can relieve PTSD-like behavior in a rodent model. We are now conducting an early-phase trial for patients with severe treatment-refractory PTSD.

Methods

This proof-of-concept two-year trial follows a randomized staggered-onset design. After baseline evaluations, the patients undergo bilateral BLn DBS electrodes placement using a transfrontal trajectory. The primary outcome measure, the clinician-administered PTSD scale (CAPS) is administered monthly. A fluorodeoxyglucose (FDG) PET scan is completed at rest and under symptomatic conditions. This PET is performed at baseline and one year post-operatively.

Results

Our first subject had a baseline CAPS score of 119 classifying him among the most severely ill patients. He had failed to improve despite 20 years of treatment with medication and psychotherapy. His baseline FDG PET study showed higher amygdala metabolic activity during symptom-provocation compared to rest. Since the initiation of DBS, his CAPS score has reduced by $40 \%$ and the occurrence of nightmares has reduced by more than $90 \%$. BLn DBS has allowed the patient to experience a broader range of positive emotions and to display more empathy. It has been well tolerated without adverse events.

Conclusion

Failure of fear extinction is the main barrier to treatment in patients with intractable PTSD. We hypothesize that BLn DBS reduces the functional output of the amygdala, thus permitting fear extinction. 


\section{MEG Coherence and DTI Connectivity in Mesial Temporal Lobe Epilepsy}

Mohammad-Reza Nazem-Zadeh, Jason M. Schwalb, Susan Bowyer, John E. Moran, Esmaeil Davoodi-Bojd, Andrew Zillgitt, Hassan Bagher-Ebadian, Fariborz Mahmoudi, Ellen L. Air, Kost Elisevich, Hamid Soltanian-Zadeh

\section{Introduction}

Magnetoencephalography (MEG) has been used to aid in the localization of epileptogenic foci in epilepsy patients by identifying highly coherent activity in epileptic neural networks. Diffusion tensor imaging (DTI) can identify virtual pathways of major fiber tracts and quantify abnormalities that are thought to reflect disruption of the microstructural environment. We aimed to correlate MEG and DTI findings in patients with mesial temporal lobe epilepsy (mTLE) and examine the ability of each modality to predict laterality in mTLE.

\section{Methods}

Preoperative resting state MEG data was analyzed to determine coherence in 54 anatomical sites in 17 adult mTLE patients who subsequently underwent surgical resection and Engel class I outcome, and compared with 12 age- and gendermatched controls. DTI tractography identified the fiber tracts passing through these same anatomical sites. Then, DTI nodal degree and laterality index were calculated and compared with the corresponding MEG coherence and laterality index.

Statistical analysis was performed with one way ANOVAs with Bonferroni adjustment.

\section{$\underline{\text { Results }}$}

MEG coherence laterality showed significant differences for right versus left mTLE in insular cortex and both lateral orbitofrontal and superior temporal gyri $(p<0.01)$. Likewise, DTI nodal degree laterality showed significant differences for right versus left mTLE in gyrus rectus, insular cortex, precuneus and superior temporal gyrus $(\mathrm{p}<0.01)$. In insular cortex, MEG coherence laterality correlated with DTI nodal degree laterality $\left(\mathrm{R}^{\wedge} 2=0.46 ; \mathrm{p}=0.003\right)$ in the cases of mTLE. None of these anatomical sites showed statistically significant differences in coherence laterality between right and left sides of the controls. MEG coherence laterality and DTI nodal degree laterality were in agreement with the declared side of epileptogenicity in $94 \%$ and $100 \%$ of the patients,

respectively.

Conclusion

MEG coherence laterality was related to the nodal degree laterality extracted by analysis of DTI connectivity. Both indices may be helpful in noninvasive lateralization of mTLE patients.

\section{Betweenness Centrality of Networks Constructed from Intracranial EEG and Surgical Epilepsy Outcomes}

\section{Bartosz T. Grobelny, Dennis London, Travis Hill, Emily}

North, Patricia Dugan, Werner Doyle

Introduction

Analysis of brain connectivity has shown promise in predicting outcomes after epilepsy surgery. Betweenness centrality (BC) offers a quantitative way to describe the importance of nodes in a connectivity network. We constructed networks from intracranial EEG (iEEG) recordings of surgical epilepsy patients and analyzed $\mathrm{BC}$ as a function of outcome.

$\underline{\text { Methods }}$

123 seizures from 36 surgical epilepsy patients who received pre-resection iEEG were included. iEEG was analyzed from periods immediately before and after seizure onset, at the midpoint of the seizure, and immediately before and after seizure termination. These samples were used to model connectivity networks at different frequency ranges using the direct transfer function. BC was calculated for every node in each network. Post-surgical Engel classification outcomes were obtained from the medical record. Using postimplantation and post-resection MRI scans, we determined resection volumes relative to the implanted electrode contacts. Statistical significance of the differences between BC means was calculated by randomly reshuffling the pooled data. Bonferroni correction was applied to $\mathrm{p}$-values, so only $\mathrm{p}$ values $<0.001$ were considered significant.

Results

The mean BC across all nodes in the post-seizure time point was significantly higher in all frequency ranges for patients who were not seizure free $(p<0.001)$. The mean BC of resected nodes was higher in the midpoint of the seizure in the 18,30 , and $50 \mathrm{~Hz}$ networks of patients with persistent seizures $(\mathrm{p}<0.001)$. The means of BC across all nodes and across nonresected nodes for this time point were not different.

Conclusion

Higher average $\mathrm{BC}$ in the post-seizure networks is associated with poor outcome regardless of resection. Also, patients with lower average $\mathrm{BC}$ of resected nodes in select frequencies at the mid-seizure had better outcomes. This difference depended only on the selection of resected nodes as the BCs of the entire population of nodes and non-resected nodes did not differ with outcome. 
Infection Rates in Trials of a Cranially Implanted Neurostimulator

Peter B. Weber, Ryder Gwinn, David W. Roberts, Richard S. Zimmerman, Ritu Kapur, Martha J. Morrell

$\underline{\text { Introduction }}$

Brain responsive stimulation using the RNS System is a new treatment for medically intractable epilepsy. Unlike other neuromodulation devices, the RNS Neurostimulator is cranially implanted, similar to a cochlear device. Sufficient prospective patient follow-up is now available to describe the long-term infection risks for the cranial implant.

Methods

All infection related serious adverse events (SAE) (requiring a procedure or hospitalization) as reported in patients

participating in RNS System trials that were not due to a seizure were identified and described as incidence per patient per year, and according to initial implant or replacement. Data cutoff was 11/01/2015.

$\underline{\text { Results }}$ 256 patients were implanted with the RNS Neurostimulator and Leads and followed for an average of 6.9 years (range 10.5 days - 11.2 years). Over a total of $1,715.2$ patient implant years, 28 patients (10.9\%) experienced 30 SAEs that were either device related or of uncertain device relation and involved an incision or implant site infection. All infections were reported as soft tissue; there were no reports of infections of meninges or parenchyma. The rate of serious infection was 0.017 per implant year, and $3.58 \%$ per neurostimulator procedure $(n=30 / 836)$. Culture results were provided for $22 / 28$ patients. Agents included staph (10), propionibacterium (5), serratia (2), pseudomonas (3), and E. coli (1). 20 patients had the neurostimulator (with or without leads) explanted. Four were subsequently reimplanted.

$\underline{\text { Conclusion }}$

The cranial implant for the RNS Neurostimulator offers several advantages over a pectoral implant. The signal to noise ratio is excellent, which is important for a responsive system, and the implant is not apparent to the patient and others, providing a cosmetic advantage. Implant related infections in the clinical trials were soft tissue and rates compare favorably to other implanted devices. Additional clinical experience will continue to identify methods by which infection risks can be further reduced.

\section{Patterns of Seizure Outcome and Recurrence After Laser Insterstitial Thermal Therapy}

Victor Du, Ashesh Mehta

Introduction

Laser interstitial thermal therapy (LITT) is gaining acceptance as an alternative to craniotomy for resection of seizure foci.

However, long term data and patterns of seizure recurrence are unavailable. We describe outcome on 28 patients undergoing laser ablation for epilepsy, including 23 patients with greater than 1 year outcome.

Methods

Retrospective chart review was performed for 28 consecutive patients with at least 3 months of followup. When documentation was unavailable, patients were contacted by telephone. Engel and ILAE Outcomes were assessed at 3, 6 month, 1 and 2 year time points.

$\underline{\text { Results }}$

One patient was lost to followup. 6 patients had hypothalamic hamartomas $(\mathrm{HH})$ and 22 had medial temporal lobe epilepsy (MTLE). While $94 \%$ of patients were seizure-free after 3 months, $15 \%$ of these patients had recurrence in the next three months. Of $79 \%$ of patients who were seizure free after the first 6 months, $13 \%$ developed seizures in the next 6 months. Only $1 / 13(8 \%)$ of patients who were seizure free after the first year had seizure recurrence in the second year. At 1 year, $70 \%$ of patients in the entire series were seizure-free and the remainder had rare seizures. At 2 years, $54 \%$ were still seizure-free, with $30 \%$ having rare seizures and the remaining $8 \%$ with worse outcome. Best Engel 1 outcomes were seen in mesial temporal sclerosis (MTS) (72\% at 1 year; $60 \%$ two years) and hypothalamic hamartoma and mesial temporal tumors (100\% at one and two years).

Conclusion

LITT demonstrates similar outcomes to those described using traditional open surgery for the treatment of epilepsy at 1 year and beyond for a variety of etiologies. Similarities between techniques apply not only to seizure-free outcome, but success rates with respect to pathology (with MTS and single lesions giving the best results) as well as patterns of recurrence. 


\section{Invasive EEG Associated Complications}

Kostas N. Fountas, Iordanis Georgiadis, Theophanis Giannis, Konstantinos Vagkopoulos, Eftychia Z. Kapsalaki

Introduction

The employment of invasive EEG monitoring via subdural electrodes is routinely employed in patients undergoing evaluation for medically refractory epilepsy, and also for stimulation and cortical mapping in patients with tumors in eloquent areas. In our current study, we present our complications from a series of adult patients.

Methods

Our study covers an 8-year period. A total of 162 (84 males, 78 females) consecutive patients undergoing invasive EEG monitoring via subdural electrodes were included. All patients underwent implantation of subdural electrodes via a craniotomy. The type and number of the implanted electrodes, the duration of monitoring, and the occurrence of any complications were prospectively recorded. All participants underwent a post-implantation brain CT and MRI, within the first 24 hours postoperatively.

Results

The patients' age ranged between 17 and 63 years (mean:35.5). Eighty-four patients underwent invasive EEG monitoring for epilepsy, 52 for primary brain tumor in eloquent cortical areas, 13 patients for a metastatic tumor, and 13 for a vascular lesion (9 AVMs, and 4 cavernous malformations). The number of the implanted electrodes ranged between 1 and 6 (mean:3.3), while the number of contacts varied from 8 to 80 (mean:43.5). The duration period ranged from 2 to 8 days (mean:3.5). Headache occurred in 59 patients $(59.9 \%)$. Brain edema was documented in 28 patients $(17.3 \%)$. An epidural hematoma was seen in 43 patients $(26.5 \%)$. A subdural hematoma was demonstrated in 17 patients $(10.5 \%)$. A temporary neurological deficit was observed in 3 patients $(1.8 \%)$. The recording of non-habitual seizure activity was recorded in 7 patients $(4.3 \%)$. There were no infections in our series, although positive cultures occurred in 13 cases $(8.0 \%)$. The cumulative complication rate was $39.5 \%$ in our current series. Conclusion

Although complications during invasive EEG may be frequent, the vast majority of them are minor, with no clinical consequences.

\section{Structural and Functional MRI Characterization of Trial Spinal Cord Stimulation Responders in Failed Back Surgery Syndrome}

Peter A. Pahapill, Guangyu Chen, Andrew Nencka, Hao Shu, Shekar N. Kurpad, Shi-Jiang Li

Introduction

Chronic back pain is a burden to society and difficult to treat. Chronification of pain may involve structural and brain network connectivity changes that include transitions from somatomotorsensory to more emotionally-related brain networks. Spinal cord stimulation (SCS) can be effective in treating failed back surgery syndrome (FBSS) patients. Despite having a successful response to a brief SCS trial (SCSr), 30-50\% of implanted patients fail to achieve satisfactory long-term pain relief. Improved pain control may be achieved with alternative, bursting patterns of SCS which may preferentially affect emotional components of chronic pain. Our goal is to begin to utilize functional imaging to help guide SCS management.

Methods

Prior to SCS permanent implant, anatomical and resting state fcMRI were performed on 10 SCSr FBSS patients, and on 16 age-matched controls. Gray matter density (GMD), brain inter-network function connection strengths (FCS) were calculated amongst motor (MTN), default model (DMN), salience (SAN), striatum (STM), temporal (TEP), hippocampus (HIP) and dorsal attention (DAN) networks. Two sample t-test with 5000 random permutation and Bonferroni correction were performed for GMD and FCS respectively. A linear regression was used to test the clinical significance of FCS.

$\underline{\text { Results }}$

Significantly decreased GMD in bilateral precentral gyri and increased GMD in HIP/PHG areas were found in the SCSr FBSS group. The inter-network FCS of STM-MTN, STMDMN, STM-TEP, STM-HIP and STM-DAN were significantly decreased in this group. The FCS of STM was negatively correlated with pain scores.

Conclusion

This is the first ever report in SCSr FBSS patients of decreased FCS between striatum and other functional networks that was also inversely correlated with pain scores, which may reflect mechanisms of pain chronification. The finding of increased HIP GMD in these patients is consistent with previous reports in animals and humans, of hippocampal neurogenesis with persistent pain. 


\section{Role of the Motor Cortex Stimulation on Neurotransmitter Concentration in the Periaqueductal Gray Area (PAG)}

Emerson Magno de Andrade, Raquel Martinez, Rosana Pagano, Patricia Lopes, Aline Auada, Ivo Lebrun, Manoel Teixeira, Erich T. Fonoff

$\underline{\text { Introduction }}$

Subthreshold stimulation of the motor cortex is a relative new technique that has been used for the treatment of patients with chronic neuropathic pain syndromes that are resistant to conventional pharmacological treatment. The motor cortex may be the most rostral structure in the neuroaxis responsible for pain modulation, and recent results obtained by our group demonstrated that motor cortex stimulation (MCS) increases the neuronal activation of periaqueductal gray (PAG) in animal models of peripheral neuropathies. The PAG is one of the main subcortical centers of the descending pain suppressor system, and receives inputs from several brain areas.

\section{Methods}

Experiments were carried out on Wistar male rats, subdivided into three surgical groups. In the first group, the right sciatic nerve was tied, producing a chronic constriction injury (CCI), the second group was the sham-operated animals, and the third group was the control non-operated animals (naïve). All the rats underwent implantation of unilateral transdural electrodes on the motor area corresponding to the right hind paw. The animals were evaluated for mechanical hyperalgesia test and a chronic microdialysis guide cannula was stereotaxically implanted into the PAG, according to the Rat Brain Atlas. The microdialyzate samples were collected and the neurotransmitters analysis was performed by a highperformance liquid chromatography (HPLC).

\section{$\underline{\text { Results }}$}

Animals subjected to CCI of the sciatic nerve showed reversal of mechanical hyperalgesia after MCS. MCS induced a significant increase in glycine levels during MCS (153\% increase) and after MCS (134\%). The GABA concentration increases $145 \%$ during transdural stimulation. Glutamate levels showed no change in PAG microdialysate after MCS. Conclusion

Our results suggest that the neurotransmitters glycine and GABA, released in PAG during MCS, contribute to descending antinociceptive actions. The results of this project will contribute for the elucidation of the mechanisms of the antinociceptive effect of MCS, a phenomenon that has not been fully understood currently.
A Study of Cognitive Function in Treatment-refractory Obsessive Compulsive Disorder Treated by Capsulotomy

Feilong Gong, Wei Wang

Introduction

Anterior capsulotomy (AC) is used as a last resort treatment for treatment-refractory obsessive compulsive disorder (OCD).Previous studies assessing neuropsychological outcomes in OCD have indicated several forms cognitive dysfunction, but few have focused on changes in cognitive function in OCD patients who have undergone surgery. $\underline{\text { Methods }}$

We selected 14 treatment-refractory OCD patients who underwent bilateral anterior capsulotomy between 2007 and 2013, 14 non-operated OCD patients and 14 healthy control subjects. The three groups were matched for gender, age and education. We conducted several neuropsychological tests in all 42 subjects, including: Similarities, Block Design-- subsets of the Wechsler abbreviated scale of intelligence (WASI), Immediate and Delayed Logical Memory, Immediate and Delayed Visual Reproduction--subsets of the Wechsler memory scale-revised (WMS-R), Corrects, Categories, Perseverative Errors,Non-perseverative errors, Errors of Wisconsin Card Sorting Test (WCST) on five separate occasions (baseline, 1 month, 3 months, 6 months and 12 months after AC. The Yale Brown Obsessive Compulsive Scale (Y-BOCS) was used to measure OCD symptoms in the 28 OCD patients.

Results

In both OCD groups, Y-BOCS scores decreased significantly during the 12-month follow-up period. The surgical group showed higher levels of improvement in verbal memory, visual memory, visuospatial skill and execution function than the control group.

Conclusion

The findings of this study suggest that anterior capsulotomy may not only reduce OCD symptoms but also positively influence cognitive deficits. 


\section{Single Unit Activity Increases in Response to Mechanical and Thermal Stimuli in Parkinson's Patients with Chronic Pain}

Youngwon Youn, Abigail Belasen, Lucy Gee, Julia Prusik, Adolfo Ramirez-Zamora, Julie G. Pilitsis

Introduction

Although known for its motor manifestations, Parkinson's disease (PD) often includes an array of pain symptoms. In fact, pain has often been reported to be a major disabling symptom and sometimes even the primary complaint. Deep brain stimulation (DBS) has recently been shown to improve chronic pain.

Methods

Ten STN cells from seven patients were isolated during micro electrode recording (MER). Mechanical and thermal detection and pain thresholds were measured pre-operatively. Stimuli were then applied at those thresholds during intraoperative MER.

$\underline{\text { Results }}$

Chronic pain patients had significantly increased neuronal firing with exposure to innocuous mechanical ( $\mathrm{p}=0.021)$, noxious pressure $(\mathrm{p}=0.012)$, and noxious thermal $(\mathrm{p}=0.002)$ thresholds. Patients with no chronic pain had no significant changes to any mechanical or thermal stimuli.

Conclusion

The present study reveals PD patients with chronic pain have significantly increased neuronal responses to mechanical and thermal stimuli as opposed to patients who do not. Our data reveals that patients suffering from pain have abnormal hyperactivity in the STN in response to stimuli, suggesting that DBS may treat chronic pain by modulating this abnormality.

\section{Deep Brain Stimulation of Frontal Lobe Behavioral Networks for Alzheimer's Disease}

Ali R. Rezai, Emily Weichart, Dylan Nielson, Jun Zhang, Punit Agrawal, Per Sederberg, Michael V. Knopp, Douglas W. Scharre

Introduction

Deep brain stimulation (DBS) of the fornix has recently been investigated for memory improvement in Alzheimer's disease (AD). While memory issues are common, executive function, behavioral regulation, decision-making, and problem solving issues result in profound functional impairments and challenges for caretakers. Given the safety of ventral anterior capsule/ striatum (VC/VS) DBS and its role in behavioral regulation, we performed a pilot trial of DBS in AD patients. Methods

The study was a FDA IDE prospective open label pilot trial of DBS compared to a matched group without DBS from the Alzheimer's Disease Neuroimaging Initiative (ADNI) cohort. Three ADNI matched AD patients underwent stereotactic VC/VS DBS implantation. The primary outcome measure was the Clinical Dementia Rating Sum of Boxes (CDR SB), a widely used global measure summating six domains of cognitive and functional performance. Serial 2-deoxy-2$\left[{ }^{18} \mathrm{~F}\right]$ fluoro-D-glucose (FDG) positron emission tomography (PET) images were also performed longitudinally over time with DBS.

Results

DBS was well tolerated without significant adverse events. Our a priori responder criterion was less decline of CDR-SB over time with DBS as compared to the ADNI Cohort. At 1824 month long-term outcome, all three AD DBS participants had less performance decline and two of them had significantly less decline $(\mathrm{p}<0.05)$ over time on CDR-SB as compared to the ADNI matched cohort. Minimal changes or increased metabolism on FDG PET were seen in frontal cortical regions after chronic DBS.

Conclusion

This is the first report of DBS in AD involving a frontal lobe surgical target implicated in behavioral regulation. Our findings of DBS safety and less decline in CDR-SB compared to a matched ADNI cohort is promising and emphasizes the need for further studies including randomized controlled trials. While the goal of attenuating memory loss receives the majority of attention in $\mathrm{AD}$ clinical trials, future research strategies should incorporate efforts to improve both cognitive, behavioral, and memory circuit deficits. 


\section{Identification of the Stria Medullaris Thalami Using Diffusion Tensor Imaging}

Robert J. Dawe, Mehmet Kocak, Sepehr B. Sani

Introduction

The lateral habenula $(\mathrm{lHb})$ has been identified in the neural circuitry of refractory major depressive disorder. The major afferent pathway to the $1 \mathrm{HB}$, the stria medullaris (SM), has been identified as a possible target for deep brain stimulation (DBS) in treatment of refractory depression. Since SM is not readily identifiable on conventional MRI, we evaluated whether diffusion tensor imaging and MR tractography can be used to identify the SM for DBS targeting.

Methods

Five patients underwent MRI studies. Patients were imaged using a 3-Tesla Siemens Verio MRI scanner. The exam included a 3D T1-weighted axial sequence (MP-RAGE, 260x227-mm FOV, 1x1x1-mm resolution, scan time of 4 minutes) and an axial diffusion-weighted sequence (8 volumes with no diffusion weighting, 69 volumes with diffusion weighting of $1000 \mathrm{~s} / \mathrm{mm} 2$ uniformly distributed in 3D space, 256x256-mm FOV, 2x2x2-mm resolution, scan time of 16 minutes). Motion and distortion correction of the raw diffusion-weighted images to match the 3D T1-weighted volume was accomplished using TORTOISE. We then used Diffusion Toolkit and TrackVis to track and visualize fibers of the SM. A spherical seed region (radius 3-4 $\mathrm{mm}$ ) was centered on the lateral habenula. An additional "waypoint" region was drawn by hand on a coronal slice to include the ipsilateral white matter of the internal capsule between the caudate and putamen. Fiber tracts passing through both regions were identified as belonging to the SM.

$\underline{\text { Results }}$

Fiber tracts of the SM were readily identified and spanned from the basal forebrain region to the contralateral $1 \mathrm{HB}$ and monoaminergic nuclei of the upper brainstem.

Conclusion

Diffusion tensor imaging can be used to identify the stria medullaris for direct targeting in DBS surgery.

\section{Pathway Selective Deep Brain Stimulation Derived from Patient-Specific Models}

Kabilar Gunalan, Bryan Howell, Yuval Duchin, Remi Patriat, Guillermo Sapiro, Noam Harel, Cameron McIntyre

Introduction

Deep brain stimulation (DBS) of the subthalamic region is an established clinical therapy for the treatment of late stage Parkinson's disease. A fundamental biophysical effect of DBS is the generation of action potentials in axons surrounding the stimulating electrode. The subthalamic region is made up of multiple axonal pathways, and it is unclear which of these pathways are directly responsible for the therapeutic benefit (or side effects) generated by DBS. The goal of this work is to quantify the specific pathways directly activated by clinical DBS settings.

Methods

A patient-specific computational model of DBS was created based on 7T MRI data. Subcortical nuclei were segmented from T1-weighted, T2-weighted, and susceptibility-weighted images. Axonal pathways representing the hyperdirect, subthalamopallidal, pallidothalamic, and cerebellothalamic pathways, as well as the internal capsule were reconstructed by conducting tractography with diffusion-weighted images. Each of the 6000 axons reconstructed were modeled as a multi-compartment cable structures. The voltage distribution generated by the DBS electrode was calculated using a finite element method; this voltage distribution was then used to stimulate the model axons; and the response of the axons to DBS was quantified.

$\underline{\text { Results }}$

Recruitment curves were created for each electrode contact to calculate the effects of different amplitudes on the activation of each pathway. The clinically-defined therapeutic stimulation setting (contact 2, 2 Volts, 60 microseconds) activated $29 \%$ of the hyperdirect, $17 \%$ of the subthalamopallidal, and $5 \%$ of the cerebellothalamic pathways. The relative proportion of activated pathways could be most effectively biased by selecting different contacts.

Conclusion

Clinically effective stimulation most likely activates multiple pathways. Preferential stimulation of different pathways is possible but is dependent upon the patient-specific anatomy, electrode position, and stimulation parameter settings. 
The Value of Microelectrode Recording in DBS: Does it Guide Us Toward or Away from Our MRI-based Target?

Zaman Mirzadeh, Tsinsue Chen, Kristina Chapple, Margaret Lambert, Rohit Dhall, Francisco A. Ponce

$\underline{\text { Introduction }}$

Microelectrode recording (MER) and test stimulation are used to refine and confirm lead placement in DBS surgery.

Advances in intraoperative imaging have provided alternative methods of verifying stereotactic accuracy and challenged the utility of MER, which has been associated with increased risk and cost. Here we examined whether MER directed DBS lead repositioning closer to or away from the preoperatively planned target.

Methods

Over a 3-year period, we identified 50 patients from our prospectively maintained database with 61 DBS leads requiring additional brain penetrations with repositioning directed by MER. After final lead placement, these patients had intraoperative CT scans fused to preoperative MR planning images to verify targeted contact coordinates. Targeted contact coordinates prior to the MER-directed repositioning were extrapolated from the final coordinates and the known offset vector.

$\underline{\text { Results }}$

In 45 of 61 cases (73.8\%), the MER-directed offset moved the lead closer to the initial plan. The mean Euclidean errors between the preoperative plan and the targeted contact coordinates before and after the MER-directed offset were 3.2 and $2.6 \mathrm{~mm}$, respectively ( $\mathrm{p}<0.001)$. Interestingly, we found an association between the targeted nucleus and the likelihood of moving toward the initial plan. In all 17 cases (100\%) targeting STN, MER directed lead repositioning closer to our initial plan, compared to 14 of $23(61 \%)$ for GPI and 14 of 21 $(67 \%)$ for VIM cases $(\mathrm{p}=0.014)$. Number of penetrations (mean 2.3), the electrophysiological indication for repositioning (recordings: $43 \%$, motor thresholds: $25 \%$, sensory thresholds: $31 \%$ ), and pneumocephalus (59\%) were not associated with likelihood of repositioning closer to the initial plan.

Conclusion

The majority of offsets based on MER data are directed closer to the initial MRI-based target, suggesting that MER corrects for stereotactic error. Improvements in stereotactic technique to deliver the lead directly to target should reduce the dependence on MER.

\section{Compensation of Functional Neurosurgeons, A National Survey}

Joshua M. Rosenow, Brian H. Kopell, Peter Konrad, Konstantin V. Slavin

Introduction

Physician compensation models continue to evolve. Many functional neurosurgical cases reimburse physicians less well than those of many other neurosurgical subspecialties. Most available compensation surveys (MGMA, NERVES, amGA) do not include significant numbers of neurosurgeons who subspecialize in functional neurosurgery. To fill in this knowledge gap, we conducted a national survey of functional neurosurgeons.

$\underline{\text { Methods }}$

An invitation to the internet-based survey was emailed to United States members of the American Society of Stereotactic and Functional Neurosurgery and the North American Neuromodulation Society. Questions included basic demographic information as well as queries about case mix and case numbers, work relative value unit (wRVU) production, total compensation, compensation models, and compensation incentives.

$\underline{\text { Results }}$

Invitations were sent to 453 individuals and 50 responses were received. Respondents were evenly distributed geographically. $86 \%$ of respondents worked in an academic medical center (evenly splt between hospital employed and not). $80 \%$ had been in practice 15 years or fewer. Functional neurosurgery cases represented over half of total case load for $56 \%$. Average amount of protected research time was $11 \%$. Mean total number of cases per year was 306 . The mean wRVU per year was 9483 . For many respondents with the highest wRVU numbers, functional neurosurgery represented less than half of total cases. Almost half were compensated on a salary+incentive basis without an academic productivity incentive as part of their compensation. For over half, compensation was somewhat or much less than their partners. $70 \%$ did not have a non-clinical compensation component. Total compensation amounts varied widely. Conclusion

This survey provides the largest and most complete dataset on compensation for functional neurosurgeons. This may be compared to existing larger surveys such as the NERVES survey. Functional neurosurgeons may use this data in benchmarking their performance. 


\section{Levodopa-Induced Changes in Cortical Phase-Amplitude Coupling in Parkinson's Disease: An EEG Study}

Andrew M. Miller, Svjetlana Miocinovic, Nicki Swann, Philip A. Starr

Introduction

Recent electrophysiological studies have suggested that Parkinson's disease (PD) is characterized by elevated neuronal synchronization in the basal ganglia-thalamo-cortical network. Invasive electrocorticography (ECoG) studies have indicated that one measure of this synchronization in motor cortex is the coupling of broadband gamma amplitude to beta phase (phaseamplitude coupling, PAC). We have recently shown that betagamma PAC can be detected in PD patients using scalp electroencephalography (EEG). In the current study, we sought to replicate this finding in a patient group with detailed clinical characterization. We hypothesized that decreased rigidity and bradykinesia in the on-medication state would be associated with a reduction in PAC.

\section{Methods}

Resting 64-channel EEG data were collected from 9 PD patients undergoing preoperative evaluation for DBS therapy, and from age-matched healthy controls. Patients were tested off and then on dopaminergic medications. Spectral power and PAC between beta and broadband gamma were calculated. Severity of motor symptoms was assessed by a movement disorders neurologist using the Unified Parkinson's Disease Rating Scale (MDS-UPDRS).

$\underline{\text { Results }}$

UPDRS-III scores were higher in the off $(\mathrm{M}=40.4)$ vs. on ( $\mathrm{M}$ $=27.6$ ) medication state. Preliminary results indicate that betabroadband gamma PAC seen in EEG channels closest to M1 (C3 and C4) was elevated in the off compared to onmedication state and in the off-state compared to healthy controls. Within-subjects decrease in PAC was associated with decrease in UPDRS items measuring bradykinesia. However, in a subset of three patients who had reduction in tremor in the on state, PAC was greater in the on-medication state, probably because tremor reduction can elevate PAC.

Conclusion

We provide further evidence that changes in cortical PAC following levodopa administration can be detected using scalp EEG. Levodopa appears to decrease PAC in rigid-akinetic PD patients, whereas levodopa may paradoxically increase PAC in patients who experience tremor reduction in the on medication state.

\section{DBS of the STN in Parkinson's Modulates the Value of Sensory Evidence}

Dennis London, Michael Pourfar, Alon Y. Mogilner

Introduction

While DBS of the STN effectively treats many of the motor symptoms associated with Parkinson's disease, it has also has been found to increase impulsive behavior. Several recent studies have suggested that patients use less evidence to make decisions. In this study, we tested this hypothesis using a task specifically designed to determine the decision bound, the maximum amount of evidence collected before making a decision. Subjects responded at will on some trials but were interrupted on other trials, presumably before they had hit the bound.

\section{Methods}

Patients with bilateral STN DBS completed multiple 30minute sessions of an auditory task, in which they listened to two simultaneous Poisson-generated click trains presented from different headphones. They were instructed to respond with the side of the most clicks. The subjects responded prior to termination of the click trains, or the click trains stopped prior to their response. Half of each session was completed with DBS off. A 9-parameter drift diffusion model was fit to each subject's decision-making. Each trial type was fit separately. This was complemented by model-free analysis. $\underline{\text { Results }}$

7 patients have been enrolled in the study and have completed 13 sessions. Enough data has been obtained from 3 patients to permit modeling, and data collection is ongoing. DBS does not have a clear effect on the number of clicks after which a decision is made (increased in 1/7, decreased in 1/7, $(\mathrm{p}<0.01)$ no change in 5/7). Instead, DBS increased parameters describing the value of sensory evidence. In each of the 3 patients, DBS increased either the variance in the value of each click or the value of clicks that were temporally close to previous clicks $(\mathrm{p}<0.05)$.

Conclusion

STN DBS appears to modulate the value of evidence instead of modulating the decision threshold. 


\section{Automatic Detection of Local Geometric Distortion in Ultra-High-Field MRI using Computational Morphometry: Importance to Stereotactic Surgery}

Jonathan C. Lau, Ali R. Khan, Keith MacDougall, Andrew G. Parrent, Terry M. Peters

Introduction

Ultra-high-field magnetic resonance images (MRI) provide improved visualization of subcortical structures that may be useful for surgical planning in stereotactic procedures.

However, the increase in field strength can result in geometric distortion. Prior studies have evaluated this problem using qualitative and quantitative measurements from manually delineated anatomical structures and landmarks defined on phantoms as well as in vivo subjects. We propose a workflow for automatic local geometric distortion detection using an image processing framework known as computational morphometry.

Methods

24 subjects obtained structural MRI scans on both the 3T and 7T scanners at our center. An image processing pipeline was developed to align 7T to 3T images for each subject, starting with rigid body, affine 12-parameter, and finally, nonlinear registration. Voxel-based displacement was calculated from the deformation fields used for nonlinear registration. Group analysis was performed in standard MNI152 space.

Results

Nonlinear registration improved our ability to align 7T to $3 \mathrm{~T}$ images. Using group and subject-specific analysis, we were able to identify regions systematically affected by geometric distortion particularly in the occipital/suboccipital, orbitofrontal, and parasagittal areas. Average displacements in the parasagittal cortex were 1.5-3.5 mm. We also noted local displacement of the thalamus in the $2 \mathrm{~mm}$ range.

Conclusion

Our automated pipeline provides a framework for assessing the extent of geometric distortion resulting from ultra-highfield structural MRI at the group and subject levels in an exploratory fashion. Displacements in subcortical structures were in the submillimeter range consistent with prior studies, except in the thalamus where more distortion was noted. Cortically, we would caution the dependence on 7T imaging for entry-point localization. The derived displacement maps can be used to prospectively evaluate subject-specific distortions that should be taken into account in stereotactic planning.
Utilizing Quantitative Susceptibility Mapping for Direct Targeting of the Subthalamic Nucleus and Globus Pallidus Internus During Deep Brain Stimulation Surgery

Jonathan J. Rasouli, Ritesh Ramdhani, Catherine Cho, Brian H. Kopell

Introduction

Deep brain stimulation (DBS) of the subthalamic nucleus (STN) and globus pallidus internus (GPi) has demonstrated efficacy in improving motor disability in Parkinson Disease (PD). The recently developed quantitative susceptibility mapping (QSM) technique, which can accurately map iron deposits in deep brain nuclei, promises precise targeting of the STN and GPi. The objective of this prospective study was to demonstrate the use of QSM to target STN/GPi effectively by correlating with classical physiologic-based targeting measures.

Methods

The precision and accuracy of direct targeting with QSM was examined in a total of 35 PD patients between 2013-2015 at the Mount Sinai Medical Center. QSM was utilized as the primary MRI method to perform direct STN and GPi-targeting on a stereotactic planning station utilizing CT/MR fusion. Intraoperative microelectrode recordings (MER) were obtained to confirm appropriate trajectory through the sensorimotor STN/GPi.

Results

There was a high-degree of correlation (>90\%) among MER and QSM in their estimations of STN/GPi-thickness. Mean STN and GPi thicknesses were $5.4 \mathrm{~mm}$ and $6.1 \mathrm{~mm}$, respectively. Kinesthetic responsive cells were found in $>90 \%$ of MER runs. The mean radial error $( \pm$ SEM) was $0.54 \mathrm{~mm} \pm$ 0.1 . Satisfactory clinical response as determined by Unified Parkinson's Disease Rating Scale (UPDRS III) was seen at one year after surgery.

Conclusion

Direct targeting of the sensorimotor STN/GPi using QSM demonstrates a high-degree of MER correlation and can be safely and easily used for DBS lead placement with satisfactory clinical response. These results imply that targeting based on QSM signaling alone is sufficient to obtain reliable and reproducible outcomes in the absence of physiologic recordings. 


\section{Analyzing the Tradeoff Between Electrical Complexity and Accuracy in Patient-Specific Models of Deep Brain Stimulation}

Bryan Howell, Cameron McIntyre

$\underline{\text { Introduction }}$

Deep brain stimulation (DBS) is effective in treating movement disorders and shows promise for treating psychiatric disorders. Computational models of DBS have begun to be utilized as tools to optimize the therapy. Despite advancements in the anatomical accuracy of these models, there is still uncertainty as to what level of electrical complexity is adequate for modeling the electrical field in the brain and the subsequent neural response to the stimulation.

\section{Methods}

We used magnetic resonance images to create a computational model of subthalamic DBS. The complexity of the volume conductor model was increased by incrementally including heterogeneity, anisotropy, and dielectric dispersion in the electrical properties of the brain. We quantified changes in the load of the electrode, the electric potentials, and stimulation thresholds of corticospinal tract (CST) axon models.

\section{$\underline{\text { Results }}$}

Incorporation of heterogeneity altered the electric potentials and subsequent stimulation thresholds, but to a lesser degree than incorporation of anisotropy. Additionally, the results were sensitive to the choice of method for defining anisotropy, with stimulation thresholds of CST axons changing by as much as $190 \%$. Traditional approaches for defining anisotropy underestimated the expected load of the stimulation electrode, which led to underestimation of the extent of stimulation. More accurate predictions of the electrode load were achieved with an alternative approach for defining anisotropy that incorporated dielectric dispersion.

\section{Conclusion}

The results of this study delineate the level of detail that is required to accurately model electric fields in the brain. These computational models will, in turn, will be used as research tools to determine more optimal stimulation strategies for improving outcomes in DBS.

\section{Radiographic Imaging for Laser Induced Thermal Therapy: An Assessment of Optimal Image Sequences for Ablation Evaluation}

Anthony Parisi, Sri Sundararajan, Rahul Garg, Eric L. Hargreaves, Nitesh Vijay Patel, Shabbar F. Danish

Introduction

Laser induced thermal therapy (LITT) is a novel, minimally invasive method of treating intracranial neoplasms. Minimal data exists on which imaging methods are ideal for postablation imaging. The purpose of this project is to determine which MRI protocol gives the most concordance in volume assessment after MRI-guided LITT for intracranial neoplasms. Methods

20 patients who received LITT at our institution were included in our study. 3 reviewers were trained for volumetric analysis. Tumors were independently demarcated by each reviewer on post-procedural axial SPGR, axial FLAIR, axial DWI, and axial GRE sequences. The volume rating data was then analyzed using Pearson Correlation Analysis between rater pairs to determine which protocol had the most concordance amongst the three raters. The closer the correlation is to 1 , the more concordant the MRI protocol was amongst the three raters.

$\underline{\text { Results }}$

Post-contrast SPGR sequences were shown to be the most concordant in our study, with an average correlation between raters of .946. DWI (correlation $=.88$ ) was the next-most concordant imaging sequence, followed by GRE (correlation = .75). Demarcation of lesions on the FLAIR sequence (correlation $=.71)$ was least concordant in our study. Conclusion

This study described for the first time the relationship between MRI protocols and imaging of patients post-LITT. Our data shows that the SPGR-post contrast protocol tends to provide the most inter-observer concordance when evaluating tumor appearance and volumetrics post-LITT, with DWI following closely behind. In summary, our data suggests that postcontrast SPGR imaging allows for the most concordant measurement of post-LITT follow up of tumor planes when patients require follow-up imaging. 


\section{A Randomized Trial on the Efficacy of Topical Anesthesia on Pain Reduction During Frame Placement for Gamma Knife Radiosurgery}

Sean Michael Duenas, Jonathan Pun, Hesham Radwan, Meredith Ackerman, Michael Schulder

\section{$\underline{\text { Introduction }}$}

Frame application for Gamma Knife Radiosurgery (GKR) may be perceived as painful by patients. Topically applied EMLA (2.5\% Lidocaine/2.5\% Prilocaine) has been used to alleviate pain caused by injection of local anesthetic before frame placement. This study was designed to assess the efficacy of EMLA for pain reduction.

\section{Methods}

This was a prospective, randomized and controlled trial approved by our IRB. 54 patients undergoing outpatient GKR for a variety of intracranial neoplasms were divided into EMLA control and Eucerin placebo groups. Prior to frame placement, EMLA or placebo was applied to the patient's forehead. Patients used a VAS to rate pain during frame fixation at four separate intervals: during frontal injections, occipital injections, frontal screw insertion, and overall discomfort. The study was designed to observe a difference of 1.0 on the VAS scale at a power of $95 \%$.

\section{$\underline{\text { Results }}$}

Groups were controlled for age and diagnosis. The VAS for front injections for EMLA vs. placebo (5.2 \pm 2.7 vs. $5.7 \pm 2.0$ respectively, $\mathrm{p}<.45)$, back injections, ( $6.5 \pm 2.2$ vs. $5.9 \pm 2.3$ respectively, $\mathrm{p}<.30)$, two front pins ( $4.6 \pm 2.7$ vs. $4.6 \pm 2.2$ respectively, $\mathrm{p}<.99)$ and overall discomfort $(\mathrm{p}<.29)$ was not significantly different. Comparison between back and front injections for EMLA (6.54 vs. 5.19 respectively, $\mathrm{p}<0.16$ ) and placebo (5.89 vs. 5.68 respectively, $\mathrm{p}<0.69$ ) did not show significant difference between group and location $(\mathrm{p}<0.21)$. $\underline{\text { Conclusion }}$

Use of EMLA did not result in significant pain reduction when used as a preoperative supplement for stereotactic frame fixation. EMLA is no longer used as part of our routine for patients undergoing GKR.

\section{Extended Glioma Resection by Prehabilitation Induced Plasticity}

Josue Moises Avecillas-Chasin, Osman Salazar, Paola Rivera, Marcos Rios-Lago, Sandra Sanchez-Casarrubios, Alvaro Pascual-Leone, Juan A. Barcia

Introduction

The extent of resection of brain gliomas has a great impact in survival of these patients. This can be limited by the presence of eloquent areas. The aim of this study is to find out if presurgical cortical electrical stimulation of the tumoral eloquent areas, coupled with intensive neurological prehabilitation, would accelerate plasticity and thus, enable a more extensive resection.

Methods

We report on five patients with gliomas involving eloquent brain areas identified by intraoperative stimulation mapping. A grid of electrodes was placed over the residual tumor, and continuous cortical electrical stimulation was targeted to the tumoral eloquent areas. The stimulation intensity was adjusted daily to provoke a mild functional impairment while the function was intensively practiced.

$\underline{\text { Results }}$

The required stimulation intensity to impair function increased progressively in all patients, and all underwent another operation 33.6 days (mean, range: 27-37) later, when the maximal stimulation voltage in all active contacts induced no functional deficit. In all cases, a substantially more extensive resection of the tumor was possible. Intraoperative mapping and functional magnetic resonance imaging demonstrated a plastic reorganization, and all previously demonstrated eloquent areas within the tumor were silent, while there was new functional activation of brain areas in nearby regions or towards the contralateral hemisphere.

Conclusion

Cortical electrical stimulation and appropriate neurological prehabilitation prior to surgery in patients with low gliomas affecting eloquent areas can help maximize tumor resection and, thus, improve survival while maintaining function. 


\section{Single Fraction Versus Fractionated Stereotactic Radiosurgery for Large Vestibular Schwannomas: Tumor Control and Clinical Outcomes}

Sussan J. Salas, Jonathan P.S. Knisely, Michael Schulder, Mark B. Eisenberg, Maged Ghaly, Karen Black, Rona Racareanu

Introduction

The objective is to compare tumor control and clinical outcomes for patients with large vestibular schwannomas

(Koos Grade 3 and 4) treated with single fraction stereotactic radiosurgery (SRS) versus fractionated stereotactic radiosurgery (FSRS).

Methods

Twenty-two patients were treated with either SRS $(n=11)$ or FSRS ( $n=11)$. For SRS, a median single dose of 12 Gy was prescribed, whereas for FSRS a total dose of 25 Gy was prescribed over 5 daily fractions. 4 patients in the SRS group (36.4\%) and 5 patients in the FSRS group (45.4\%) had undergone prior resection. Mean tumor volume was $3.04 \mathrm{cc}$ in the SRS group and $3.77 \mathrm{cc}$ in the FSRS group.

Results

Radiological growth control was achieved in $91 \%$ of patients undergoing SRS: 8 tumors (73\%) demonstrated either a decrease in tumor volume or no change, and 2 tumors (18\%) demonstrated arrested growth after a transient period of enlargement. 1 patient required resection secondary to tumor growth and clinical progression 36 months after SRS. For patients undergoing FSRS, 100\% radiological growth control was achieved: 7 tumors $(64 \%)$ demonstrated either a decrease or no change in tumor volume, with 4 tumors $(36 \%)$ demonstrating arrested growth after transient enlargement. Radiological features including loss of central enhancement, cystic degeneration, and brainstem signal changes were not significantly different between both groups. Loss of serviceable hearing was observed in only 1 patient in the SRS group. Trigeminal sensory dysfunction developed in 1 patient in the SRS group 34 months after treatment (improved after resection) and in 1 patient in the FSRS group 19 months after treatment. 1 patient in the SRS group developed mild facial weakness (House-Brackmann Grade II) 12 months after treatment, and another patient developed facial spasms 4 months after treatment.

Conclusion

SRS and FSRS for large vestibular schwannomas achieve comparable tumor control with similar rates of neurological function preservation, even in light of prior resection. FSRS may be a good option to treat patients for whom fractionation may offer neuroprotective benefits.
Stereotactic Robotic Assisted MRI Guided Laser Thermal Ablation of Radiation Necrosis and High Grade Glioma in the Posterior Cranial Fossa

Diem Kieu Thi Tran, Alvin Chan, Sumeet Vadera

Introduction

Laser interstitial thermal therapy (LITT) is a minimally invasive procedure used to treat a variety of intracranial lesions. Utilization of robotic assistance with stereotactic procedures has gained attention due to potential for advantages over conventional techniques. We report the first case where robot-assisted MRI-guided LITT was used to treat radiation necrosis in the posterior fossa, specifically within the cerebellar peduncle.

Methods

This patient is a 60-year-old male with a history of anaplastic astrocytoma that was treated via Cyberknife radiosurgery oneyear prior. He did well after the procedure for 11 months but then began to have symptoms consistent with radiation necrosis, which was confirmed via MRI. The location and form of the lesion precluded surgical excision but made the patient an appropriate candidate for LITT. The required trajectory for LITT was too low for arc-based stereotactic navigation, thus the ROSA robot (MedTech) was utilized. Using preoperative MRI acquisitions, the lesion in the posterior fossa was targeted. Bone fiducials were required for accurate registration and we obtained an intraoperative CT image that was then fused with the MRI by the ROSA robot. We placed the laser applicator and then ablated the lesion with MRI guidance.

Results

There were no complications and the patient tolerated the procedure well with immediate improvement.

Conclusion

Here we present a case where the use of a stereotactic robot allowed the surgeon to perform LITT using a trajectory that would be extremely difficult with conventional arc-based techniques. 


\section{Robot Assisted Stereotactic Laser Ablation for a Hypothalamic Hamartoma}

Nicholas James Brandmeir, Vinita Acharya, Michael D. Sather

$\underline{\text { Introduction }}$

Hypothalamic hamartomas $(\mathrm{HH})$ are benign growths that can cause intractable gelastic seizures as well as multiple other endocrine disturbances. Seizures secondary to $\mathrm{HH}$ are often responsive to surgical resection, open resection and endoscopic resection of $\mathrm{HH}$ remains a morbid operation with relatively poor seizure control rates. Stereotactic laser ablation (SLA) has been shown to be an effective technique for ablation of $\mathrm{HH}$ and treatment of gelastic seizures. Robot assisted stereotactic techniques are gaining acceptance, but are relatively rare and have not been reported for accessing an HH.

\section{Methods}

A relevant case report is presented.

\section{$\underline{\text { Results }}$}

This is a report of a 63-year-old man who suffered from gelastic seizures secondary to an $\mathrm{HH}$. He had failed Gamma Knife radiosurgery to the lesion two times. He was treated with robot assisted SLA. After, this the patient was seizure free. He also experienced a $10 \mathrm{~kg}$ weight loss, but remained overweight at his follow up visit.

Conclusion

Robot assisted stereotaxy is an effective and safe way to target hypothalamic lesions. SLA can be an effective method to treat gelastic seizures and $\mathrm{HH}$ that have failed radiosurgery. This is the first report of the use robot assisted stereotactic techniques to treat an $\mathrm{HH}$. It is also the first report of SLA for $\mathrm{HH}$ in an elder adult. SLA of an $\mathrm{HH}$ can result in unintentional weight loss.

\section{Magnetic Resonance-Guided Laser Ablation for Postradiosurgery Metastatic Recurrence or Radiation Necrosis: Institutional Experience}

\author{
Robert N. Hernandez, Purvee D. Patel, Shabbar F. Danish
}

Introduction

Enhancing lesions that demonstrate progression after radiosurgery for metastatic brain tumors are often tumor recurrence or radiation necrosis. Magnetic resonance-guided laser-induced thermal therapy (LITT) is a minimally-invasive treatment option for postradiosurgery recurrence or radiation necrosis. We report the largest prospective series to date of LITT for the treatment of recurrent enhancing lesions after radiosurgery for brain metastases.

Methods

Patients with recurrent metastatic intracranial tumors or radiation necrosis who had previously undergone radiosurgery and had a Karnofsky performance status of $>70$ were eligible for LITT. The primary endpoint was local control using MR scans at intervals of $>4$ weeks. Additionally, we report overall survival, perioperative complications, and 30-day readmission rates.

$\underline{\text { Results }}$

30 patients (ages 46-90 years) who underwent 36 procedures for placement of 40 laser probes in 39 enhancing lesions were available for follow-up. The mean pre-procedure lesion size measured $3.45 \mathrm{~cm}^{3}$ (range $0.23-10.52 \mathrm{~cm}^{3}$ ). At a median follow-up of 42.7 weeks (range 4.9-181.9 weeks), local control was $74.4 \%$ (30 of 39 lesions), and overall survival at 6 , 12,18 , and 24 months was $83.3 \%, 46.9 \%, 23.3 \%$, and $13.3 \%$, respectively. 11 perioperative complications were encountered: 2 patients with known seizure disorders seized, 1 patient who underwent 2 procedures on different dates developed confusion in both instances, 4 patients had new or increased extremity motor weakness, 1 patient developed aphasia, 1 patient reported visual hallucinations, and 1 patient developed a facial nerve palsy. 6 patients were readmitted to the hospital within 30-days of the LITT procedure: 2 patients with known seizure disorder after seizing, 1 patient for atrial fibrillation with rapid ventricular rate, 2 patients with abdominal pain found to have ileus, and 1 patient for altered mental status. There were no procedure-related mortalities. Conclusion

LITT is an effective and safe treatment option for local control of recurrent metastatic brain disease that has failed radiosurgery. 


\section{Decoding Decision Outcomes from Single Realizations of Lateral Prefrontal Cortex Ensemble Activity}

Chadwick Boulay, Adam J. Sachs

$\underline{\text { Introduction }}$

Neurons in the lateral prefrontal cortex (LPFC) encode sensory and cognitive signals, as well as commands for goal directed actions. This brain region might be a good target for the implantation of electrode arrays as part of a cognitive neuroprosthesis. We previously demonstrated that visually guided saccade targets could be decoded from singlerealizations of pre-saccadic LPFC neuronal activity. In this work we examine the neuronal representation of the task and task acquisition, and we decode decision outcomes independent of stimulus information.

\section{Methods}

We recorded neuronal spiking activity from microelectrode arrays implanted in area 8A of the LPFC of two adult macaques while they made saccades to one of a pair of presented targets. The rewarded target was indicated by a colour cue and we changed periodically the association between colour and rewarded direction. We modeled the ensemble activity and performed a machine-learning analysis on the neural trajectories to predict reward location or intended saccade goal.

\section{Results}

Behavioural performance was poor at the onset of each new cue-target rule. Each monkey's performance improved rapidly as he learned the new rule. Models representing latent structure in the neural ensemble were mostly stable but varied slightly during learning of a new rule. The rewarded target predictions improved as the monkey learned the task, and in some instances the predictions outperformed the monkey himself. Intended saccade goals were predicted with good accuracy, especially when the peri-saccade neuronal activity was included in the model.

Conclusion

The results from the model estimations suggest that the latent structure in the neural ensemble participates in the decisionmaking process and exhibits constrained plasticity in response to changing demands. Neural prostheses that exploit this latent structure may be able to generalize well across tasks and sessions.

\section{Restoration of Functional Hand Movements in a Human with Quadriplegia Using a Cortically Controlled Functional Electrical Stimulation Device}

Ammar Shaikhouni, Marcia Bockbrader, Chad Bouton, Nicholas Annetta, David Friedenberg, Gaurav Sharma, Bradley Glenn, Austin Morgan, Milind Deogaonkar, Per Sederberg, Jerry Mysiw, Ali R. Rezai

Introduction

Neural interfaces (NI) devices connect cortical signals to assistive technology to restore lost function in paralyzed individuals. Previous work has shown that paralyzed humans are able to use NI devices to control various assistive devices such as computers, robots, and wheelchairs. Here we show that neural signals recorded from primary motor cortex can be used to control functional electrical stimulation of paralyzed hand muscles thereby allowing a chronically paralyzed human to utilize his hand in functional motor tasks.

Methods

A 24-year-old male with C5 AIS-A with zone of partial preservation to $\mathrm{C} 6$ bilaterally underwent implantation of a 96channel microelectrode array into the arm area of his left primary motor cortex. Neural signals were recorded while he imagined right arm and hand movements. The neural activity was decoded in real-time and used to activate forearm muscles using a custom-built neuromuscular electrical stimulation system.

$\underline{\text { Results }}$

Utilizing this system, the participant was able to complete functional tasks relevant to daily living that he was previously unable to complete. Objective manual muscle test strength improved from $\mathrm{C} 6$ to $\mathrm{C} 7-\mathrm{C} 8$ level, his gross grasping ability improved from $\mathrm{C} 7-\mathrm{C} 8$ to $\mathrm{C} 8-\mathrm{T} 1$ level, and his prehensile skills improved from $\mathrm{C} 5$ to $\mathrm{C} 6$ level.

Conclusion

The system presented here is the first example of an NI device used in a paralyzed human to bridge a disconnected cotricospinal tract by connecting motor cortical neural activity to muscle activation. This device offers hope for movement and independence restoration to the many patients living with paralysis. 


\section{Patient-Specific Models of Local Field Potentials Recorded from Deep Brain Stimulation Electrodes}

Nicholas Maling, Scott F. Lempka, Cameron McIntyre

$\underline{\text { Introduction }}$

Emerging innovations in deep brain stimulation (DBS) are attempting to utilize the recording of local field potentials (LFPs) as biomarkers of the disease state. However, scientific details on the biophysical origin of LFP signals remains elusive, and little is known about how the patient's unique brain anatomy and electrode placement impact the recording of such signals. Therefore, we developed a framework to theoretically analyze LFP recordings from clinical DBS electrodes that can be customized to individual patients. Methods

We created a patient-specific reconstruction of the subthalamic nucleus (STN) using MRI data and a-priori knowledge of STN neuronal density. This virtual STN was used to define the parameters of the finite element volume conductor model, and to dictate the locations of neuronal current sources in an anatomically realistic way. We then analyzed the impact of explicitly representing the DBS electrode within our LFP recording model system. Finally, we evaluated the role of distributing populations of highly synchronous neurons within the STN volume on the recorded LFP signal.

\section{$\underline{\text { Results }}$}

Incorporating patient-specific STN boundaries resulted in measureable changes to LFP amplitude compared to a spherical STN shape, particularly when one of the recording contacts was on or near the boundary of the STN. We also found that electrode contact size, recording configuration, and filtering effects have a substantial impact on the amplitude and frequency content of the recorded signal. Neuronal density in the area surrounding the electrode had a graded effect on LFP amplitude, while a more profound effect was generated by varying the synchrony of spatially discrete populations of neurons near the electrode.

Conclusion

Patient-specific details can generate quantitative differences in LFP signals and these effects can be accurately represented with appropriately parameterized models.

\section{Spontaneous, Transient Adenosine Monitoring in Parkinson's Disease Patients Using Paired-pulse Voltammetry During Deep Brain Stimulation Neurosurgery}

Seungleal Paek, Aiyana D. Batton, Luis J. Lujan, Dong Pyo Jang, Christopher J. Kimble, Kevin Bennet, Kendall H. Lee

Introduction

Adenosine plays a neuroprotective role by inhibiting neuronal activity and modulating neurotransmission. It has been shown that tremor attenuation due to DBS is associated with a spontaneous accumulation of adenosine, which activates A1 receptors and ultimately depresses excitatory transmission in the thalamus. Recent studies using rodent models also suggest that spontaneous, transient adenosine release in the prefrontal cortex and caudate could play a major role in rapid local modulation. Here, we show human spontaneous, transient adenosine-like release in the human caudate during DBS neurosurgery using paired-pulse voltammetry (PPV).

Methods

Carbon fiber tipped microelectrodes were introduced into the caudate of a cohort of patients undergoing frame-based stereotactic DBS neurosurgery for Parkinson's disease. Neurochemical detection was performed using PPV at the carbon fiber microelectrodes. PPV was applied at $5 \mathrm{~Hz}$ using a $400 \mathrm{~V} / \mathrm{s}$ sweep rate from $-0.4 \mathrm{~V}$ to $1.5 \mathrm{~V}$ back to $-0.4 \mathrm{~V}$ with respect to a reference electrode. This study was approved by the Mayo Clinic IRB.

$\underline{\text { Results }}$

Spontaneous, transient adenosine-like signatures were observed when the recording electrode was placed in the caudate. PPV enhanced the recording of these signals by mitigating the extracellular environmental changes, including $\mathrm{pH}$ shift, that normally obscure neurochemical detection. No complications occurred due to PPV recording in any patients. Conclusion

Previous studies have shown a long-term modulatory effect of adenosine with tremor attenuation during DBS. The results presented here show spontaneous transient adenosine-like signals for the first time in the human brain during DBS surgery. These dynamic transient adenosine-like signaling could be responsible for rapid, local modulation. Lastly, we also demonstrate the capability of wireless PPV monitoring for the investigation of DBS mechanisms of action. 


\section{Rate and Complications of Epilepsy Surgery in North America: Analysis of Multiple Independent Databases}

John David Rolston, Dario J. Englot, Robert C. Knowlton, Edward F. Chang

Introduction

Epilepsy surgery is under-utilized, but recent studies reach conflicting conclusions regarding whether epilepsy surgery rates are currently declining, increasing, or remaining steady. However, data in these prior studies are biased toward highvolume epilepsy centers, or originate from sources that do not disaggregate various procedure types.

\section{Methods}

All major epilepsy surgery procedures were extracted from the Centers for Medicare and Medicaid Services Part B National Summary Data File and the American College of Surgeons National Surgical Quality Improvement Program. Procedure rates, trends, and complications were analyzed, and patientlevel predictors of postoperative adverse events were identified.

\section{$\underline{\text { Results }}$}

Between 2000-2013, 6200 cases of epilepsy surgery were identified. Temporal lobectomy was the most common procedure (59\% of cases), and most did not utilize electrocorticography (63-64\%). Neither temporal nor extratemporal lobe epilepsy surgery rates changed significantly during the study period, suggesting no change in utilization. Adverse events, including major and minor complications, occurred in $15.3 \%$ of temporal lobectomies and $55.6 \%$ of hemispherectomies.

\section{Conclusion}

Our findings suggest stagnant rates of both temporal and extratemporal lobe epilepsy surgery across U.S. surgical centers over the past decade. This finding contrasts with prior reports suggesting a recent dramatic decline in temporal lobectomy rates at high-volume epilepsy centers. We also observed higher rates of adverse events when both low- and high-volume centers were examined together, as compared to reports from high-volume centers alone. This is consistent with the presence of a volume-outcome relationship in epilepsy surgery.
Impact of Insular Morphology on Feasibility of Long-Axis Cannulation for Stereoelectroencephalographic Recording

Michael J. Lang, Chengyuan Wu, Ashwini Dayal Sharan

$\underline{\text { Introduction }}$

Long-axis cannulation of the triangular borders of the insula offers distinct advantages for stereoelectroencephalography (SEEG) interrogation of possible insular epilepsy. Variable morphology of the insular surface and its orientation relative to other cranial structures dictates the feasibility of this approach. This study seeks to demonstrate the morphologic variables associated with success or failure of long-axis insular cannulation.

\section{$\underline{\text { Methods }}$}

Insular morphology was evaluated bilaterally on de-identified magnetic resonance imaging (MRI) from 100 patients without intracranial pathology from the Open Access Series of Imaging Studies (OASIS) database. Analysis was performed using volumetric, T1-weighted MRIs images were converted into AC-PC coordinate frame. Coordinate positions were taken defining the insular triangle (anterior, posterior and inferior insular points), insular apex, and planes defining superior sagittal (SSS) and transverse sinuses (TS).

\section{$\underline{\text { Results }}$}

Geometric properties of the insula were calculated, including area of insular surface, angle of the insular plane relative to sagittal plane, and relative position of the insular centroid to the mid-commissural point. Dihedral angles of the insular triangle relative to the SSS and TS were defined, as was the center of the insula triangle relative to the midcommissural point. Insular cannulation trajectories that crossed the SSS or TS were identified.

\section{Conclusion}

Insular morphology can be utilized prior to SEEG trajectory planning to identify the feasibility of long-axis cannulation. These data can potentially be used as the basis for automated or semi-automated trajectory planning software in the future to increase the ease of planning these difficult trajectories. 
Intraoperative MRI in Resective Epilepsy Surgery for Peri-eloquent Cortex Cortical Dysplasias and Heterotopias in Pediatric Subjects

Chima Oluigbo, Matthew Sacino, Cheng-Ying Ho, Jonathan Murnick, William Gaillard, John S. Myseros, Robert F. Keating

Introduction

The most important factor in seizure freedom following surgery for lesional epilepsy in peri-eloquent cortex is completeness of resection. The objective of this study was to investigate the role of intraoperative magnetic resonance (iMRI) as a complement to existing epilepsy protocol techniques, and compare rates of seizure freedom and neurological deficit in pediatric patients undergoing perieloquent lesion resections.

\section{Methods}

We retrospectively reviewed the medical records of pediatric subjects who underwent surgical resection of FCD or heterotopia localized to eloquent cortex regions at the Children's National Health System between March 2005 and August 2015. Subjects were grouped into two categories depending on whether they underwent conventional resection $(\mathrm{n}=18)$ or iMRI-assisted resection $(\mathrm{n}=11)$.

$\underline{\text { Results }}$

At the time of the last postoperative follow-up, 9 of the 11 patients $(82 \%)$ in the iMRI resection group were seizure free (Engel Class I), compared to 7 of the 18 patients (39\%) in the control resection group $(\mathrm{p}=0.05) .10$ of the $11(91 \%)$ patients in the iMRI cohort achieved complete gross resection, compared to 8 of $18(44 \%)$ patients in the conventional resection cohort $(\mathrm{p}=0.02)$. One patient in the iMRI-assisted resection group underwent successful reoperation at a later date for residual dysplasia, compared to 7 patients in the conventional resection cohort (with 2/7 achieving complete resection). Four (27\%) of the patients in the iMRI cohort developed postoperative neurological deficits, compared to 15 $(83 \%)$ patients in the conventional resection cohort $(\mathrm{p}=0.02)$.

Conclusion

Our results suggest that in comparison with conventional surgical protocol and technique for resection of epileptic lesions in peri-eloquent cortex, the incorporation of iMRI led to elevated rates of complete gross resection and postoperative seizure freedom as well as a reduction in rates of postoperative neurological deficit.

\section{Minimally-Invasive Corpus Callosotomy Technique and Outcomes: Stereotactic Radiofrequency and MR-Guided Laser Ablation}

\author{
Ashley K. Ralston, James Tao, Peter C. Warnke
}

$\underline{\text { Introduction }}$

We compared stereotactic radiofrequency (RF) and laser ablation as minimally invasive alternatives to open surgery for performing corpus callosotomy in patients with intractable epilepsy, presenting primarily with drop attacks.

Methods

Retrospective review of corpus callosotomy performed using stereotactic RF or MR guided laser interstitial thermal therapy (LITT) with the Visualase Thermal Therapy system between February 2014 and April 2015. We correlated outcome with volumetric analysis of ablative lesions.

$\underline{\text { Results }}$

Ablation of the anterior two thirds of the corpus callosum was performed in all 3 patients. Laser ablation required 2

trajectories, whereas 4 trajectories were necessary for both RF ablations. Drop attacks ceased in the laser ablation patient and one of the RF ablation patients. The other RF ablation patient continues with weekly drop attacks, significantly decreased from multiple daily drop attacks. There were no complications with either technique. Achievable lesion volume was significantly larger with interstitial laser ablation.

Conclusion

Minimally invasive corpus callosotomy, performed using stereotactic laser and radiofrequency ablation, provides seizure reduction while reducing the risks from open craniotomy. Further studies, with larger cohorts and long term follow-up, will be helpful in determining safety and long term efficacy. Real-time MRI monitoring of laser ablation provides a slight advantage and should be considered in epileptic patients with a significant proportion of atonic seizures or drop attacks. 
Insular Triangulation Technique: A Novel Stereo EEG Technique for Investigation of Insular Lobe Epilepsy

Michael J. Lang, Chengyuan Wu, Ashwini Dayal Sharan

\section{Introduction}

Insular seizure onset is a frequent cause of failed surgery for presumed temporal lobe epilepsy. Intra-cranial EEG monitoring of presumed insular lobe epilepsy is technically challenging. Previously reported strategies of trans-sylvian depth electrode placement and trans-opercular stereo EEG (SEEG) trajectories risk injury to the middle cerebral artery branches. We describe a technique for long-axis SEEG cannulation along the triangular boundaries of the insular cortex.

Methods

As part of larger implants for seizure onset localization, twelve patients were implanted with insular SEEG electrodes using the triangulation technique with robotic assistance. Electrodes were implanted to approximate the cortex along the sylvian triangle, such that the superior insular electrode spans the posterior to anterior insular points, the inferior electrode from the posterior to inferior insular points, and the anterior electrode from anterior to inferior insular points.

$\underline{\text { Results }}$

Technical success was achieved in all patients, with 5 to 9 active insular contacts per electrode. There were no procedural complications related to electrode placement. In three patients, insular onset was discovered, resulting in tailored partial insulectomy. At three month follow up, two patients achieved Engel Class 1A seizure freedom, while the third has Engel Class 1B outcome with persistent epigastric aura.

\section{Conclusion}

The insular triangulation technique allows broad investigation of the insula, spanning functional subdivisions of the insular lobe, and permits tailored partial insulectomy.

\section{The Electrocorticogram and Connectivity Dynamics Before and After Corpus Callosotomy follows Lateralization of Seizure Foci}

Victor Du, Pierre Megevand, Erin Yeagle, Jose Herrero, Miklos Argyelan, Ashesh Mehta

Introduction

Rapidly propagating frontal lobe seizures that are difficult to lateralize may do so after corpus callosotomy. We performed callosotomy using laser interstitial thermal therapy (LITT) in three patients who were undergoing stereoelectroencephalography (SEEG) and examined both the electrocorticogram electrophysiology and neuroimaging connectivity measures.

Methods

SEEG electrodes were placed three patients with suspected underlying laterization. All three patients underwent anterior corpus callosotomy using stereotactic laser interstitial ablation with electrodes in place. Resting fMRI, diffusion tractography were obtained one week before electrode implantation and one week after. In addition, the resting electrocorticogram and corticocortical evoked potential mapping were performed before and after the callosotomy procedure using the same electrodes that were rigidly held in place with skull bolts, resulting in minimal electrode migration after callosotomy. Results

All patients had marked lateralization of both interictal activity as well as seizure onset. Alterations in interhemispheric connectivity was reliably demonstrated using DTI and resting fMRI. This was paralleled by a similar changes in resting electrocorticography and corticocortical evoked potentials over areas with projections to the area of the callosum that was lesioned. Both intrahemispheric connectivity as well as interhemispheric connectivity beyond the callosotomy were relatively maintained as reflected by electrocorticographic- and MRI-based measures.

Conclusion

Callosotomy using LITT may be used in conjunction with SEEG to accurately identify ictal onset in cases where seizure lateralization is difficult. Our results further confirm the correspondence of MRI- and electrophysiological-based connectivity measures by showing that alterations of function connectivity occur using both methods after surgical disconnection. 


\section{The Impact of Mirth-Inducing Ventral Striatal Deep Brain Stimulation on Functional and Effective Connectivity}

William Gibson, Shinho Cho, Osama Abulseoud, Krzysztof R. Gorny, Joel Felmlee, Kirk M. Welker, Bryan T. Klassen, Paul Hoon-Ki Min, Kendall H. Lee

Introduction

Deep brain stimulation (DBS) of the ventral capsule/ventral striatum (VC/VS) is an investigational therapy for treatmentresistant obsessive-compulsive disorder. The ability of VC/VS DBS to evoke spontaneous mirth in patients, often accompanied by smiling and laughter, is clinically well documented. However, the neural correlates of DBS-evoked mirth remain poorly characterized.

Methods

Patients undergoing VC/VS DBS surgery underwent intraoperative evaluation in which mirth-inducing and nonmirth-inducing stimulation localizations were identified. Using dynamic causal modeling (DCM) for fMRI, the effect of mirth-inducing DBS on functional and effective connectivity among established nodes in limbic corticostriato-thalamo-cortical (CSTC) circuitry was investigated. $\underline{\text { Results }}$

Both mirth-inducing and non-mirth-inducing VC/VS DBS consistently resulted (conjunction, global null, familywise error-corrected p-value < 0.05) in activation of amygdala, ventral striatum, and mediodorsal thalamus. However, only mirth-inducing DBS resulted in functional inhibition of anterior cingulate cortex. Dynamic causal modeling revealed that mirth-inducing DBS resulted in an attenuation of effective connectivity from both anterior cingulate and mediodorsal thalamus to ventral striatum relative to non-mirth-inducing stimulation.

\section{Conclusion}

These results suggest that DBS-evoked mood elevation is accompanied by distinct patterns of limbic thalamocortical connectivity. Using the novel combination of DBS-evoked mood alteration and functional MRI in human subjects, we provide new insights into the network-level mechanisms that influence affect.

\section{Does VNS Treat Pseudoseizures? An Analysis of Vagus Nerve Stimulation in Patients with Epilepsy and Co- Morbid Psychogenic Nonepileptic Seizures}

\author{
Andrew C. Vivas, Hena Waseem, Christian Reitano, Fernando \\ L. Vale
}

Introduction

Patients with intractable epilepsy may suffer from co-morbid psychogenic nonepileptic seizures (PNES). The efficacy of vagus nerve stimulation (VNS) for epilepsy is well established, but its impact on PNES is unknown. The goal of this study is to determine whether VNS leads to an improvement in psychogenic episodes in patients with comorbid disease.

Methods

All patients who underwent VNS at our institution were included. Video EEG results were reviewed and patients who demonstrated both epileptic seizures and PNES were identified. These patients were contacted and queried for data regarding preoperative and post-operative non-epileptic seizure burden. Post-operative quality of life $(\mathrm{QoL})$ was evaluated using the QOLIE-31 questionnaire.

$\underline{\text { Results }}$

518 patients underwent VNS for epilepsy in the period from 1998 to 2015. 16 patients were found to carry concomitant diagnoses of epilepsy and PNES. Of this subset, 13 patients completed a survey administered by the research team. The remaining patients could not be contacted or were lost to follow up. 10 of the 13 patients reported a $25-50 \%$ improvement in their psychogenic episodes after VNS implantation and 11 of the 13 patients had improved quality of life as measured using the QOLIE questionnaire.

Conclusion

Patients with epilepsy and co-morbid PNES benefit from VNS. It is unclear whether the benefit is conferred strictly from decreased epileptic seizure burden. The possible effect on PNES may be related to the known effect of VNS on depression and mood. Further studies are necessary to elucidate the role of VNS and other forms of neuromodulation in the treatment of psychiatric disease. 
An Easily Implemented, Open Access Semi-Automatic Pipeline for Intracranial Electrode Localization

Timothy G. Dyster, Yagna Pathak, Elliot Smith, Sameer A. Sheth

$\underline{\text { Introduction }}$

Intracranial electrode implantations for DBS and seizure localization are common functional neurosurgical procedures. In order to refine therapeutic targets and draw meaningful conclusions from electrophysiological data, intracranial electrodes need to be accurately localized. The demand for simpler and more efficient methods to localize implanted electrodes has grown as the volume of these procedures continues to increase. We developed a semi-automated pipeline that integrates pre-operative MRI and post-operative CT data to determine electrode locations.

\section{Methods}

The semi-automated pipeline was tested with a sample of patients $(n=9)$ who underwent implantation of sEEG, surface grid/strip, or a combination of these electrode configurations. We co-registered the pre-op MRI with the post-op CT (3D Slicer, Boston, MA) to align electrodes in patient-specific anatomy and extracted a volumetric brain image (FSL, Oxford, UK) to create a mask that represented the dataspace of interest (MATLAB, Natick, MA). The image was processed using size and intensity thresholding, erosion, and Gaussian kernel convolution. We determined coordinates for electrodes' centroids, which allowed for sophisticated visualization. Coordinate accuracy was evaluated by comparison to coordinates generated from a validated manual method for electrode localization.

\section{$\underline{\text { Results }}$}

Coordinates for approximately 600 contacts on the implanted electrode were successfully computed for all patients.

Visualization demonstrated that automation did not affect the number of electrodes detected. Coordinates were compared to output from a validated manual method for electrode

localization, and accuracy was non-inferior.

Conclusion

The method described is an accurate and easily-implemented method for intracranial electrode localization using MATLAB and open access software. Compared to similar open access methods, our pipeline requires minimal user input, which significantly reduces person-hours required for task completion. From a clinical perspective, this pipeline allows for seamless retrospective analysis of surgical targets and thus has the potential to inform prospective image-guided surgical protocols.
A Semi-Automated Software for Estimating Subthalamic Nucleus Boundaries and Assisting Optimal Target Selection for Deep Brain Stimulation Implantation Surgery

John Thompson, Hagai Bergman, Steven Ojemann, Adam Olding Hebb, Salam Oukal, Aviva Abosch

\section{Introduction}

Deep brain stimulation (DBS) of the subthalamic nucleus (STN) has become standard of care for the treatment of Parkinson's disease. Reliable interpretation of microelectrode recording (MER) data, used to guide DBS implantation surgery, requires expert electrophysiological evaluation. We have developed a software package, implemented on an electrophysiological recording system, to provide online objective estimates for entry into, and exit from, STN. In addition, the optimal electrode track for DBS implantation into STN can be determined through this software's ability to detect changes in beta-spectrum activity.

\section{$\underline{\text { Methods }}$}

We retrospectively collected data from 8 MER-guided STNDBS surgeries (4 neurosurgeons, 3 sites), in which estimates for entry into and exit from STN, and optimal implant track selection, were compared between those determined by the software, and those by the implanting neurosurgeon.

\section{$\underline{\text { Results }}$}

Comparison between software and neurosurgeon differed by $0.02 \mathrm{~mm} \pm 1.90 \mathrm{~mm}$ (mean $\pm \mathrm{SD}$ ) for STN entry, and -0.24 $\mathrm{mm} \pm 1.31 \mathrm{~mm}$ for STN exit, with negative indicating below target. Agreement between software and neurosurgeon for optimal track selection was $87 \%$ (7 of 8 surgeries).

\section{Conclusion}

These data demonstrate that the software can reliably and accurately estimate entry into and exit from STN, and select the optimal track for DBS implantation. A larger sample size will provide stronger validation of results. 


\section{Robot-Assisted Placement of Depth Electrodes Along the Long Axis of the Amygdalohippocampal Complex}

Diem Kieu Thi Tran, Alvin Chan, Sumeet Vadera

$\underline{\text { Introduction }}$

Classically, transoccipital hippocampal depth electrode implantation requires a stereotactic headframe and arc and the patient to be placed in a seated or prone position, which can be cumbersome to position and uncomfortable for the surgeon. Robotic intracranial devices are increasingly being utilized for stereotactic procedures such as stereolectroencephalography (SEEG) but commonly require patients be placed in headneutral position to perform facial registration. Here we describe a novel robotic implantation technique where a stereotactic intracranial robot is used to place bilateral hippocampal depth electrodes in the lateral position.

\section{Methods}

Four patients underwent SEEG depth electrode placement, which included placement of bilateral hippocampal depth electrodes. Each patient was positioned in the lateral position and registered to the robot with laser facial registration. Trajectories were planned with the robotic navigation software, which then identified the appropriate entry points and trajectories needed to reach the targets. After electrode implantation, target localization was confirmed using computed tomography.

\section{$\underline{\text { Results }}$}

Electrodes targeting the amygdalohippocampal complex were accurate and there were no complications in this group. An average of seven electrodes were placed per patient. Ictal onset was localized for each patient. All patients subsequently underwent temporal lobectomy; at 6 months, 3 patients were seizures and the 4th had a significant reduction in seizures burden.

\section{Conclusion}

We have developed the Robot-Assisted Lateral Transoccipital Approach, which is an advantageous technique for placing bilateral amygdalohippocampal depth electrodes using robotic guidance. Benefits of this technique include fewer electrodes required per patient and ease of positioning compared with seated or prone positioning.

\section{Model-Based Battery Longevity Estimates for a Deep Brain Stimulation Pulse Generator}

Alexa Schlein, Bob Ozawa, G.Karl Steinke

Introduction

While power usage is a minor concern for rechargeable Deep Brain Stimulation (DBS) devices, it remains a consideration while programming non-rechargeable DBS devices, as increased power usage will generally lead to decreased battery longevity. Newly available directional leads may add to these concerns, as the higher impedances of the smaller electrodes, coupled with the opportunity to selectively stimulate from one segmented electrode, could increase power usage. While the power of the output waveform may be calculated theoretically for a DBS device, internal power use and battery longevity depend on details of the device design. This study was carried out to compare model-predicted DBS battery longevity under various programming scenarios, including the use of directional leads with smaller, higher-impedance electrodes. Methods

A model was developed and tested to characterize the power usage and battery characteristics of the Boston Scientific Vercise PC Implantable Pulse Generator. The model takes into account the details of the design of the Vercise PC IPG, program settings, and impedances to estimate device power usage. The model was applied to a range of programming settings and electrode impedances. This model will be used to estimate battery longevity for a variety of programming scenarios.

$\underline{\text { Results }}$

Battery longevity estimates for the Vercise PC system, including scenarios with use of the directional lead, will be reported.

Conclusion

Battery longevity can be affected by changes in programming. Battery longevity can be extended in some cases with the use of current steering on multiple electrodes. 
Clinical Outcome and Location of Active Contacts in the Centromedian Thalamic Nucleus Deep Brain Stimulation in Refractory Epilepsy

Byung-Chul Son

$\underline{\text { Introduction }}$

To investigate the clinical outcome and location of active contacts in chronic centromedian nucleus (CM) deep brain stimulation (DBS) for refractory epilepsy.

Methods

The outcome of CM stimulation was evaluated with percent (\%) seizure reduction compared to the baseline three months. To determine the location of active contacts, 27 leads in 14 patients with refractory epilepsy were studied. An analysis was conducted to determine whether any coordinates of the center of the active contacts predicted percent seizure reduction.

\section{Results}

With an average follow-up of $18.2 \pm 5.6$ months, the mean percent seizure reduction $(\mathrm{n}=14)$ was $68 \pm 22.4 \%(25-100 \%)$. Eleven of 14 patients $(78.6 \%)$ could achieve $>50 \%$ improvement in the frequency of seizure. Specifically, all four patients (100\%) with generalized epilepsy (Lennox-Gastaut syndrome) and seven out of 10 patients (70\%) with multilobar epilepsy showed $>50 \%$ reduction in seizure frequency. The mean coordinates of center of the active contact were located in the superior part of anterior ventrolateral CM. The calculated coordinates of laterality from midline (x), anteriorposterior (y) and height (z) from posterior commissure (PC) did not correlate with seizure outcome measured by percent seizure reduction. However, the locations of active contacts used during chronic CM stimulation in multilobar epilepsy were identified more ventral to those used in generalized epilepsy.

\section{Conclusion}

Chronic CM stimulation is a safe and effective means in the treatment of refractory epilepsy.

\section{Prognostic Predictors in Non-Selective Amygdalohippocampectomy for Refractory Mesial Temporal Epilepsy}

Alexandra Santos, Clara Romero, Jose Cabral

\section{Introduction}

Mesial temporal lobe epilepsy is the most common cause of refractory epilepsy in adults. In these patients selective or nonselective amygdalohippocampectomy can lead to seizure freedom in $62-70 \%$ of cases. However, the prognostic factors determining seizure outcome are still debatable. The purpose of this study was to identify the pre and post operative factors that are independent predictors of seizure outcome of patients who underwent non-selective amygaladohippocampectomy in Epilepsy Surgery Group from Centro Hospitalar de Lisboa Ocidental.

Methods

Case-control study of the patients who underwent nonselective amygdalohippocampectomy, in our center, between 1997 and 2015. Patients were divided in two groups according do Engel classification- seizure free (Engel class I) and non seizure free (Engel Classes II- IV). Univariate analysis was performed to analyse the potential prognosis predictors, including demographic characteristics, epilepsy history, imaging findings, pathology results and post surgical data. Results

During this period 119 patients underwent non-selective amygdalohippocampectomy. After an average 9 years followup (range 1-18 years) 91 patients (76\%) were seizure-free and 28 patients (24\%) were non seizure free 18 patients Engel Class II, 7 patients Engel Class III and 3 patients Engel Class IV. In univariate analysis 4 factors were associated with nonseizure free outcome: date of surgery before $2005(\mathrm{p}=0.02)$, negative $\mathrm{MRi}(\mathrm{p}=0.02)$, major surgical complications $(\mathrm{p}=0.0001, \mathrm{OR} 13.04)$ and seizures in early post-operative period $(\mathrm{p}=0.032)$. No significant differences were found in gender, age at the time of surgery, epilepsy duration, surgery side and epilepsy history.

Conclusion

In our series, negative pre-operative MRi, epilepsy group low experience, major surgical complications and seizures in early post-operative period were associated with higher risk of non seizure-freedom after non-selective amygdalohippocampectomy. 


\section{Optogenetic Suppression of Epileptic Activity Using a Chemically Activated Luminopsin}

\section{Jack Tung, Thomas Shiu, Kevin Ding, Robert E. Gross}

Introduction

Optogenetics has shown great promise as a direct neuromodulatory therapy for halting seizure activity in various animal models of epilepsy. However, light delivery into the brain is still a major practical challenge that needs to be addressed before future clinical translation. Not only does light delivery into the brain require surgically implanted hardware that can be invasive, but it is also difficult to illuminate large or multiple structures due to light scatter and attenuation. We have bypassed the challenges of external light delivery by directly coupling a bioluminescent light source to an inhibitory opsin as a single fusion protein, which we term an inhibitory luminopsin (iLMO). iLMO2 was previously shown to inhibit neural activity in response to both external light illumination and a chemical substrate, coelenterazine (CTZ).

Methods

The ability of iLMO to suppress seizure activity was tested in the context of two acute models of epilepsy. Acute focal discharges were induced by injection of bicuculline into the dorsal hippocampus of rats while acute generalized seizures were induced by intraperitoneal injection of pentylenetetrazol (PTZ). The ability of iLMO2 to suppress epileptic discharges was tested by expressing iLMO2 in pyramidal cells of CA1 and CA3 and injecting CTZ mixed with bicuculline through a chronically implanted cannula-electrode. The ability of iLMO2 to suppress behavioral seizures was investigated by expressing iLMO2 in the granule cells of the dentate gyrus and/or the anterior nucleus of the thalamus and administering CTZ before an intraperitoneal injection of PTZ.

Results

iLMO activation in the hippocampus was shown to acutely suppress bicuculline-induced epileptic discharges, increase the time to seizure onset, and reduce the maximum discharge frequency. Optogenetic inhibition of granule cells of the dentate gyrus and/or the anterior nucleus of the thalamus with iLMO2 was able to reduce the total seizure duration and mortality associated with systemic PTZ administration. Conclusion

We have developed and applied a novel optogenetic probe that is capable of non-invasive, hardware-independent inhibition of neural activity that will add to the versatility, scalability, and practicality of utilizing optogenetic approaches for halting seizure activity in vivo.

\section{One-Year Outcome of Magnetic Resonance-Guided Stereotactic Laser Amygdalohippocampotomy for Mesial Temporal Lobe Epilepsy}

Matthew A. Stern, Jon Timothy Willie, Robert E. Gross

Introduction

Stereotactic magnetic resonance imaging-guided laser amygdalohippocampotomy (SLAH) is a minimally invasive approach to surgical treatment of drug-resistant mesial temporal lobe epilepsy (MTLE). Although short term outcomes in two small series have been reported (Willie et al., 2014; Kang et al., 2016), one year outcomes represent the gold standard by which to compare SLAH to standard open temporal surgery such as anterior temporal lobectomy (ATL) or selective amygdalohippocampectomy (SAH), a recent meta-analysis of which demonstrated one-year seizure freedom rates of $75 \%$ and $67 \%$, respectively. The subset with mesial temporal sclerosis (MTS) yielded higher rates $(78 \%$ verses $71 \%$, respectively). Here we present a consecutive series of 41 patients with MTLE that underwent SLAH. Methods

All 41 patients with MTLE (25 of whom had MTS) who underwent SLAH at our institution and who had follow-up $=12$ months were included. Surgical outcome was stratified by Engel's classification (class 1 = free of disabling seizures). Results were retrospectively analyzed as a non-inferiority trial with the objective performance criterion $(\mathrm{OPC})=67 \%$ (equivalent to $\mathrm{SAH}$ ), and the non-inferiority margin (f) $=8 \%$ (= absolute risk reduction between ATL and SAH). The subgroups with and without MTS were similarly analyzed (OPC $=71 \%, \mathrm{f}=7 \%$ ).

$\underline{\text { Results }}$

At one-year following SLAH 56.1\% (95\% CI $\pm 15.2 \%)$ of all MTLE patients and $64.0 \%(95 \% \mathrm{CI} \pm 18.8 \%)$ of MTS patients were free of disabling seizures (Engel 1) suggesting statistical non-inferiority to SAH. Fewer $(43.8 \%$, CI $95 \% \pm 24.3 \%)$ of non-MTS patients were seizure-free.

Conclusion

In this largest single center experience to date, SLAH was non-inferior to $\mathrm{SAH}$ at one year, with superior results in patients with MTS. Compared to open temporal lobe surgery, SLAH is an effective minimally invasive alternative with a favorable neurocognitive profile. In the minority of patients that do not achieve seizure freedom, SLAH presents no barrier to additional open surgery. 
Temporoparietooccipital Disconnection in Epilepsy Surgery: Case Series

\section{Alexandra Santos, Clara Romero, Jose Cabral}

Introduction

Surgical techniques for the treatment of refractory hemispheric epilepsy evolved from ressection to disconnection techniques, maintaining efficacy with less morbidity. In multilobar subhemispheric epilepsy the disconnection techniques, although recently described, are also been used more frequently, but their efficacy in epilepsy control and safety have only been seldom described. The aim of this study is to evaluate the outcome and morbidity of patients who underwent temporoparietooccipital disconnection in Epilepsy Surgery Group from Centro Hospitalar de Lisboa Ocidental. Methods

Retrospective analysis of patients treated in our centre who underwent temporoparietooccipital disconnection. Data collected included demographic characteristics, epilepsy history, imagiologic features, surgical data, pathology results, seizure outcome according to Engel classification and complications.

Results

Ten patients underwent temporoparietooccipital disconnection, between 2010 and 2015. Six patients were male and 4 were female, with median age of 5.5 years at time of surgery (range 2-52 years). Patient's median age of seizure onset was 1.4 years (range $0.2-7$ years) and median duration of epilepsy before surgery was 3 years (range 1.8-46 years). Six patients underwent right side surgery and 4 left side posterior disconnection. Pathology results were available for 4 patients: 2 ischemic lesions and 2 cortical dysplasias. 1 patient died during follow-up and 3 patients underwent hemispherotomy for seizure recurrence. The seizure outcome of the other 6 patients was Engel I in 4 patients (Ia in two, Ib in one and Ic in one), Engel IIIa in one patient, and Engel IVB in one patient, after a median follow-up of 41 months (range 8-57 months). Morbidity was verified in only one patient, who had a malignant brain swelling that required craniectomy and remained in vegetative state after surgery.

Conclusion

Temporoparietooccipital disconnection is a safe technique and effective in seizure control that can spare motor function in carefully selected patients.
The Development of an Automated Single Pulse Electrical Stimulation Protocol to Identify the Seizure Onset Zone in Focal Epilepsy

Tyler Davis, Paul A. House

Introduction

Identification of the seizure onset zone (SOZ) is a crucial step in the surgical care of patients with partial epilepsy. The SOZ is usually identified through manual analysis of intracranial electrode recordings during spontaneous seizures. Although this approach is the "gold standard", it is costly, timeconsuming, and imperfect. Single pulse electrical stimulation (SPES) has been explored as a means of SOZ identification, but previous SPES protocols are unsuitable for clinical care. We are developing a novel SPES technique to identify the SOZ in an automated and reproducible manner.

Methods

SPES is applied to all implanted electrodes using a pseudorandom routine with multiple repetitions. Trialaveraged spectrograms that are masked for significance $(\mathrm{p}<0.05)$ are used to assign values to each electrode based on response strength and time-frequency pattern. Maps are generated from these values to provide an estimate of the SOZ.

$\underline{\text { Results }}$

We have developed a unique protocol of SPES application and analysis over the last 2 years. We first explored low-level stimulation parameter space, using $4 \mathrm{~mm}$ circular subdural electrodes, in multiple patients, and found unique responses in the SOZ required a stimulation intensity of $3.5 \mathrm{~mA}$ using $1 \mathrm{~ms}$ pulses. We then identified two unique response patterns in 5 patients with focal epilepsy. Stimulation of electrodes over the clinically determined SOZ showed suppression of broadband $(10-250 \mathrm{~Hz})$ power lasting as long as 1 second. In one patient, an alternate pattern of broadband burst in power $150-400 \mathrm{~ms}$ after stimulation was shown. Response maps developed from these feature sets for all 5 patients demonstrate high specificity for the SOZ. Our SPES protocol does not induce afterdischarges or seizures.

Conclusion

Our results, while preliminary, are encouraging. Identification of the SOZ is rapid, reproducible, and specific, which suggests that our approach, if validated, may be useful for clinical care. 


\section{Insular Laser Ablation for Focal Onset Seizures in Children, Early Experience}

David J. Donahue, Angel Hernandez, Cynthia Keator, Saleem Malik, M. Scott Perry

$\underline{\text { Introduction }}$ Insular resection for epilepsy entails significant risk of injury to eloquent cortex, critical insular surface vessels, adjacent opercula, the internal capsule and basal ganglia. Few surgical series reporting outcomes after surgery for insular epilepsy in children exist. We report our early experience with minimally invasive stereotactic insular laser ablation in 6 children diagnosed with insular-onset seizures.

\section{$\underline{\text { Methods }}$}

Over the past 20 months, the senior author has performed 140 epilepsy surgical procedures, including invasive monitoring, cortical resection, and laser ablation, on 105 children to address focal onset seizures (VNS procedures are not included in this total). Of 32 children undergoing laser ablation for focal onset epilepsy, 6 ( 2 boys, 4 girls) who received stereotactic laser insular ablation after workup suggested insular onset seizures are the subject of this presentation. Age at seizure onset ranged from 2.5 to 9 years (average 3.92). Age at time of surgery ranged from 4 to 18 years (average 12). 3T MRI was normal in 3 children prior to surgery. Four had undergone prior resective craniotomy and/or invasive monitoring. Platform for directing the laser fiber (Visualase) was either the Leksell stereotactic frame or the ROSA robotic system. Patients were observed in the neuro ICU overnight. Most were discharged the following day (average length of stay 1.3 days).

\section{$\underline{\text { Results }}$}

Four children achieved Engel Class I outcome at follow-up 1, 9, 13, and 19 months respectively (one is off all anticonvulsants); 1 patient obtained Engel Class III at followup 7 months; 1 patient achieved Engel Class 4 at 7 months. No child sustained a new neurologic deficit related to the ablation procedure; two of the children received ablations of the dominant hemisphere.

Conclusion

Stereotactically directed laser ablation provides a promising alternative to open resection for children suffering insular onset seizures.

\section{Homonymous Hemianopsia as a Complication From Laser Interstitial Thermal Therapy for Mesiotemporal Epilepsy}

Walter J. Jermakowicz, Michael E. Ivan, Ramses Ribot, Bruno Wieckowski, Naymee Velez-Ruiz, Enrique Serrano, Andres Kanner, Jonathan R. Jagid

Introduction

Laser interstitial thermal therapy (LITT) is a novel tool that is quickly emerging as a mainstream therapy for temporal lobe epilepsy (TLE). We present the case of a 24-year-old male who developed homonymous hemianopsia as a result of LITT for TLE. Detailed anatomical comparisons are provided between this patient and 17 prior TLE patients treated with LITT at our institution in an attempt to better-understand this complication.

Methods

This is a retrospective chart review of all patients that have undergone LITT for TLE at our institution. Detailed volumetric tracings of mesial temporal lobe structures were performed for all patients using high-resolution pre- and postop MRI scans. For all patients, volumes of hippocampus, amygdala, ablation zone and CSF dorsal to the hippocampus were calculated.

$\underline{\text { Results }}$

The 24-year old male woke up with a complete right-sided homonymous hemianopsia after LITT of left mesial temporal structures. By five months post-op he had partial recovery of vision in the central superior quadrant. MRI suggested inadvertent ablation of the lateral geniculate nucleus. Comparison of intra-op images suggests the patient's laser catheter was not positioned significantly higher on the craniocaudal axis than in the prior patients. Similarly, ablation number, energy delivered and size were not significantly greater for this patient compared to the prior patients. However, the subject did have significantly smaller CSF spaces dorsal to the hippocampus, compared to the prior patients.

Conclusion

Homonymous hemianopsia is a complication of LITT for TLE that patients with small CSF spaces dorsal to the hippocampus may be susceptible to. Our data suggest that inspection of these structures on pre-op MRI scans may help identify this subset of patients. When such anatomy is identified we recommend lowering laser catheter trajectory and decreasing ablation power through the hippocampal body and using additional temperature set points at the lower thalamic border. 
The Rate of Visual Field Deficits Following Laser-Ablation Amygdalo-Hippocampectomy in Patients with Mesial Temporal Lobe Epilepsy

Dali Yin, Steven Ojemann, Cornelia Drees, Aviva Abosch

$\underline{\text { Introduction }}$

As a novel, minimally invasive surgical technique, laser ablation of epileptogenic foci has become an alternative to open surgery for patients with mesial temporal lobe epilepsy (MTLE). Visual field defects (VFDs) are reported to occur in $52-100 \%$ of patients following open surgery for MTLE.

However, the rate of VFD following laser ablation amygdalohippocampectomy (AHE) in patients with MTLE is unknown. Methods

We performed a retrospective case series of 3 consecutive patients who underwent laser interstitial thermal therapy (LITT) AHE, for medically refractory MTLE between June and December 2015. Follow-up included assessment of seizure outcome and VFDs.

$\underline{\text { Results }}$

All 3 patients underwent formal visual field testing at 3 months postoperatively ( 1 male; 2 female). The mean age was 53.3 years (range 28 - 69 years). All 3 patients had preoperative video EEG, MRI, fMRI, DTI and tractography, PET, Wada test and neuropsychological evaluation. During follow-up ranging from 5 - 7 (median 5.7) months, all patients achieved significant seizure reduction, and one patient was seizure free. Postoperatively, no patient reported subjective visual changes or had detectable VFDs based on bedside confrontation testing. Formal visual field testing was normal in these three patients. No other complications or neuropsychological side effects related to laser ablation were noted.

\section{$\underline{\text { Conclusion }}$}

Short-term follow-up in our series suggests that laser ablation AHE is associated with a lower rate of VFD. Interestingly, it did not cause VFDs in any of our patients. A larger series, with longer follow-up is being acquired, and is necessary to assess the robustness of this finding.

\section{Intraoperative CT and NexFrame Guided Placement Bilateral Hippocampal Based Responsive NeuroStimulator for Bilateral Mesial Temporal Sclerosis}

\author{
Kunal Gupta, Ahmed M. Raslan
}

Introduction

Patients with medically refractory epilepsy are often referred for neurosurgical intervention, including surgical resection, or open loop stimulation such as vagal nerve stimulation. For bilateral hippocampal sclerosis, resection is typically unilateral due to severe cognitive deficits associated with bilateral resection. Studies have demonstrated the use of subdural electrodes to establish laterality of the majority of seizures, followed by unilateral resection, however patients often have recurrence. We therefore placed a closed loop responsive neurostimulator device, and report on technical aspects, outcomes and electrographic recordings.

Methods

A 44 y.o. Caucasian male was referred to the neurosurgical service for bilateral mesial temporal sclerosis and medically refractory epilepsy. He underwent extensive EEG assessment and bilateral ictal onset was noted, with no clear laterality. Bilateral mesial temporal electrodes were placed using the Nexframe and intra-operative CT. His ictal activity was recorded post-operatively and correlated to his seizures, allowing electrocorticographic correlation of each individual seizure subtype.

$\underline{\text { Results }}$

This patient endorsed 5 different seizure semiologies, including simple partial seizures, complex partial seizures, and 3 further subtypes he classified as "jolts", auras, and a feeling of dissociation. Using the implanted responsive neurostimulator, we obtained bilateral temporal electrocorticography and noted rhythmic high amplitude activity correlating with each seizure type. In terms of laterality of his events, $51.4 \%$ were left sided, $15.0 \%$ were right sided and $33.6 \%$ were indeterminate.

Conclusion

Our experience with a responsive neurostimulator device, using intra-operative CT and Nexframe guided placement suggests that this novel described method is safe and accurate. We obtained highly accurate electrocorticography related to both mesial temporal lobes, and correlated this with the patient's seizure semiology, an unprecedented level of analysis permitted by implantation of a closed-loop system. Such systems potentially herald tailored epilepsy management for individual patients. 


\section{Deep Brain Stimulation for Thalamic Pain Syndrome with Additional Lead for Dystonia}

Mario Zanaty, Marshall Holland, Royce Woodroffe, Jeremy D.W. Greenlee, Chandan G. Reddy

Introduction

Thalamic pain syndrome is known to be refractory to medications and difficult to control. We present the first case of thalamic pain syndrome associated with dystonia that was treated with deep brain stimulation (DBS).

Methods

This is a case report of a 60-year-old female who suffered a right posterior cerebral artery stroke after aneurysm clipping complicated by thalamic pain syndrome which developed in a delayed fashion. This was manifested by severe left-sided face and body allodynia and hyperesthesia, and was associated with marked left-sided dystonia. Her symptoms were progressive and refractory to conservative management. The pain was disabling and debilitating. After multidisciplinary discussion, the patient underwent stereotactic right-sided lead implantation in the ventral capsule/ventral striatum for pain and right-sided stereotactic lead in the globus pallidus interna for dystonia, with microelectrode recording. A single impulse generator (IPG) was implanted. The leads placement and the IPG placement were performed in a single stage under general anesthesia.

Results

The surgery was successful without complications. She had marked improvement in her dystonia, with successful programming. Her affective component of pain improved subjectively but required multiple programming session and adjustment over time. She was able to reduce her pain medication consumption. The patient was satisfied with the results and her surrounding reported marked improvement and re-integration to society. She continued however to have intermittent breakthrough spells of severe pain. Unfortunately, she died from small cell lung carcinoma a year after her DBS surgery.

Conclusion

Deep brain stimulation targeting multiple networks is feasible and safe. It seems feasible and successful for pain management but would require multiple programming sessions.

\section{Novel Test of Affective Bias Is Modulated by Both Subcallosal Cingulate and Amygdala Deep Brain Stimulation}

Kelly R Bijanki, Christopher K. Kovach, Helen S. Mayberg, Cory S. Inman, Andrea L. Crowell, Robert E. Gross, Jon Timothy Willie

Introduction

Brain stimulation is a viable therapy as well as an important method for mapping brain functions. With application of deep brain stimulation (DBS) to psychiatric disorders, sensitive measures are needed to quantify effects of stimulation on emotional processing. We examined effects of stimulation to two limbic regions, the subcallosal cingulate (SCC) and the amygdala, on 'affective bias', the tendency to interpret external stimuli in a manner consistent with one's current emotional state.

Methods

A novel affective bias task was developed to quickly and covertly measure emotional state. Over 4-6 minutes, patients rate the intensity and valence of images of emotional facial expressions. We examined stimulation effects in two groups: patients with treatment-refractory depression undergoing SCC DBS therapy, and epilepsy patients undergoing amygdala stimulation via stereo-EEG electrodes during inpatient intracranial monitoring.

Results

Three SCC DBS patients showed significant positive shifts in affective bias with chronic DBS therapy $(\mathrm{t}=-2.296, \mathrm{DF}=179$, $\mathrm{p}=0.023$ ). Furthermore, two DBS patients showed a rapid negative shift in bias following acute (18 minutes) discontinuation of chronic stimulation $(\mathrm{t}=-2.330, \mathrm{DF}=53$, $\mathrm{p}=0.024$ and $\mathrm{t}=2.88, \mathrm{DF}=59, \mathrm{p}=.006$ respectively). Likewise, six epilepsy patients show significantly positive shifts in affective bias with acute amygdala stimulation $(\mathrm{t}=-4.753$, $\mathrm{DF}=5, \mathrm{p}=.005$ ).

Conclusion

Affective bias shows rapid, significant changes with stimulation at two critical nodes of the limbic system, suggesting utility as an emotional outcome measure in brain stimulation studies. This task may facilitate tracking and predicting treatment response in DBS targeting limbic structures. Future studies will determine whether affective bias can predict possible neuropsychiatric complications in patients undergoing mapping of brain circuitry ahead of resections. 


\section{Deep Brain Stimulation for Children with Intractable Secondary Dystonia: 3 Cases}

Douglas Anderson, Jacquelyn P. Hill, Kurt Grahnke, Xabier Beristain, Mary Keen

$\underline{\text { Introduction }}$

Deep brain stimulation (DBS) of the globus pallidus interna (GPi) has been used in the treatment of intractable dystonias in the pediatric population and presently carries a humanitarian device exemption (HDE). While effective in the treatment of primary generalized dystonia, it has been more challenging to assess the initial and long term effects of DBS for secondary dystonia, especially in children. In addition to pain and loss of neurological function, children with severe and intractable secondary dystonia often face a degree of cognitive impairment not seen in primary dystonia. We have performed GPi DBS on three pediatric patients with intractable secondary dystonia refractory to medicines who experienced regression in functional neurological status and persistent pain .

\section{$\underline{\text { Methods }}$}

Our patients were evaluated by developmental pediatric specialists and referred for evaluation of surgery because of painful secondary dystonia associated with decreases in motor function. In conjunction with a movement disorders neurologist, we obtained Investigational Review Board (IRB) approval for this study. For those receiving surgery, DBS leads are placed under general anesthesia into the posterior medial GPi with neuronavigation and CRW frame. We used micro-electrode recording to supplement anatomical localization of the targets. Electrodes were implanted in one or two stages, followed by separate surgeries for each pulse generator/battery. Patients are evaluated post-operatively using the Burke-Fahn-Marsden Dystonia Rating Scale (BFMDRS) and follow-up clinic visits. Also, we sought observations via interviews from parents and teachers of the children, of functional changes observed.

$\underline{\text { Results }}$

We have documented sustained but fluctuating improvement in motor function and spasticity. Changes in electrode settings were managed by the movement disorder neurologist. There have been no complications to date. Parent and teacher observations and notes and the BFMDRS have been additional sources of information.

Conclusion

We believe DBS-GPi represents a useful treatment modality for carefully selected cases of severe intractable dystonia with neurological regression. The BFMDRS may not fully or adequately characterize the full benefit noted by patient, parents, and teachers.
Accuracy Index of Minimally Invasive MRI-Guided Stereotactic Laser Amygdalohippocampotomy

Mark Russell Witcher, Alaine Keebaugh, Robert E. Gross, Jon Timothy Willie

$\underline{\text { Introduction }}$

MRI-guided Stereotactic Laser Amygdalohippocampotomy (SLAH) for mesial temporal lobe epilepsy may reduce collateral injury and cognitive morbidity normally associated with open temporal lobe surgery. Given the long trajectories and narrow anatomical corridors required for the safe and effective application of this therapy, however, accurate device placement and trajectory control is critical. Notably, the accuracies of various stereotactic methods reported in the literature may be for relatively shorter trajectories (e.g. biopsies, deep brain stimulation), necessitating a standardized assessment of accuracy relative to trajectory length. To assess the stereotactic accuracy of an MRI-compatible percutaneous skull-mounted miniframe (ClearPoint ScalpMount and SmartFrame, MRI Interventions, Irvine, CA) designed to accommodate minimally invasive access (twist drill craniostomy) while maintaining accuracy at both the target and entry point, we have defined the 'accuracy index', a proposed measure of $2 \mathrm{D}$ target accuracy relative to trajectory length.

Methods

We utilized a miniframe to perform 41 procedures targeting the amygdalohippocampal complex for laser ablation in an interventional MRI suite. Trajectory planning and stereotactic navigation were completed at time of procedure and accuracy metrics were assessed postoperatively using the ClearPoint workstation.

$\underline{\text { Results }}$

The miniframe facilitated a stab incision and $3.2 \mathrm{~mm}$ twist drill craniostomy (improving over a previous version that necessitated a larger incision and craniostomy) for SLAH. At mean trajectory length from bone outer table to target of $109.45 \pm 8.29 \mathrm{~mm}$, the mean coronal $2 \mathrm{D}$ radial error was 0.98 \pm 0.7 [Accuracy index $=0.0087$ ]. The mean Euclidian distance between the initially planned and actual scalp entry point was $2.66 \pm 1.4 \mathrm{~mm}$.

Conclusion

The miniframe accommodates minimally invasive MRIguided stereotactic neurosurgical procedures while affording accuracy at both scalp entry and the intended intracranial target. Performing the entire procedure in the MRI suite provides immediate visualization of target anatomy and recognition of deflections or other sources of inaccuracy. 
Findings of the MER in STN Target Through the Parietal Approach for DBS Implant

Angelo R. C. Azevedo, William Omar Contreras Lopez, Luiz Batista, Marcelo Pereira, Erich Talamoni Fonoff

Introduction

We present a 61 years-old female patient, with a 23 years history of Parkinson's disease, who underwent implantation deep brain stimulation in the subthalamic nucleus (STN). The patient unfortunately developed one year after surgery a bilateral subcutaneous and skin infection at the insertion in the scalp. She was treated with multiple antibiotic therapy presenting partial improvement and it was necessary to remove the leads.

$\underline{\text { Methods }}$

Stereotactic surgery to redeploying the leads was proposed due to the quality of life improvement achieved after DBS, however in order to avoid the same entry point or any vicinity to the formed infected area, the parietal access was proposed to target the STN. Electrophysiological mapping was performed bilaterally. Microelectrode recording showed a loss of signal which we believe corresponded to the former space occupied by the first electrode.

$\underline{\text { Results }}$

Significant improvement in UPDRS motor score (63\%) was obtained with bilateral stimulation 4 months after re-operation using one contact of the lead, versus UPDRS motor score of $46 \%$ after previously DBS implanted.

Conclusion

Parietal access is a viable option to access the STN in postinfection cases and may even become a primary entry point for implantation of DBS electrodes in the STN since it may allow a major STN stimulation area.
Optimization of MR Guided Focused Ultrasound Parameters and Location for the Treatment of Essential Tremor and Tremor Dominant Parkinson's Disease: A Comprehensive and Comparative Analysis of 60 FUS Thalamotomies for ET and PD

Aaron E. Bond, William Wagstaff, Nader Pouratian, W. Jeffrey Elias

Introduction

Transcranial MR guided focused ultrasound (MRgFUS) thalamotomy has been proposed as a new treatment for essential tremor and Parkinson's disease. The optimal parameters and technique used during the sonication process is currently under investigation for this new technology. We reviewed the procedural data from three clinical trials of thalamotomy to correlate patient outcomes with acoustic parameters, thermal dose, MRI and probabilistic DTI. Methods

Patient outcomes were assessed using CRST scores and UPDRS scores. Acoustic parameters included the number of elements, energy, time, and number of sonications. Thermal dose was estimated using 240 cumulative effective minutes at $43^{\circ} \mathrm{C}(240 \mathrm{CEM} 43)$ and achievement of a minimum of $54^{\circ} \mathrm{C}$ for at least $3 \mathrm{~s}$ as indicated by MR thermography. DTI was used to identify the areas of maximum thalamocortical connections to the pre-central gyrus. Overlap of lesions with DTI maps was performed with Matlab.

Results

A linear relationship exists between the predicted lesion diameter and the actual. POD1 lesion sizes were $40 \%$ and $28 \%$ of predicted based on MR thermography maps of temperature thresholds and 240CEM43 thresholds respectively. 1 month actual vs. predicted was $26 \%$ independent of the predicted model. Errors in the predicted size is driven by $2 \mathrm{D}$ thermometry scanning error which can be as high as $1 \mathrm{~mm}$. Lesion volume and patient outcomes did not correlate owing to variability of lesion overlap with the DTI maps. ET Patients had improved tremor scores following treatment at 1 year vs. PD patients (75\% improvement vs. $41 \%)$.

Conclusion

Transcranial MRgFUS thalamotomy has demonstrated tremor improvement in ET and PD; but there is variability in the outcomes and durability. Optimal acoustic parameters, position, and thermal dose are critical. Intra-procedural modeling can improve and predict the lesioning process with less error. Application of DTI for Vim targeting could refine lesion placement and improve patient outcomes. 
LINAC Radiosurgery for Glomus Jugulare Tumors: Retrospective Cohort of 23 Patients

Lior Ungar, Roberto Spiegelmann

$\underline{\text { Introduction }}$

Glomus jugulare tumors (GJTs), also referred to as paragangliomas, are rare, slow-growing, highly vascular tumors that usually arise within the jugular foramen of the temporal bone. Until recently, surgery was the treatment of choice for glomus jugulare tumors. However, in the last decade, radiosurgery has arisen as a promising alternative treatment providing an excellent tumor control with a low risk of treatment-related cranial nerve injury.

\section{Methods}

Between 1994 and 2013, 30 patients with GJTs were treated in our institution using linear accelerator (LINAC) SRS either as primary or complementary treatment. Seven patients were lost for follow-up. Comprehensive clinical follow-up for retrospective analysis was available for 23 patients (median age 64 years, range 18 - 87 years) with median follow-up of five years (range 1 - 10 years). In 19 patients, LINAC-SRS was the primary treatment, in 4 cases surgery ( 2 cases) or embolization ( 2 cases) preceded radiosurgery. The median dose to tumor margin was $14 \mathrm{~Gy}$ (range $12.5-27.5 \mathrm{~Gy}$ ) and the median tumor volume was $5.25 \mathrm{ml}$ (range $0.5-15.4 \mathrm{ml}$ ). $\underline{\text { Results }}$

Following the LINAC-SRS treatment, 14 of 23 patients (60\%) showed improvement of previous neurological deficits, whereas 9 patients (40\%) remained unchanged. At the end of follow up, tumor shrinkage was seen in 13 patients and a stable volume in 8 (91\% tumor control rate). Two cases of tumor progression were observed: one of the patients underwent a second LINAC-SRS and the other patient underwent open resection. No radiation related complications were observed during the follow-up.

Conclusion

LINAC SRS was an excellent treatment option for GJTs, achieving high rate of long term tumor control and satisfactory neurological improvement with no treatment-related complications.
A Comparison of Outcomes Between Deep Brain Stimulation (DBS) Under General Anesthesia Versus Conscious Sedation with Awake Evaluation

François Alesch, Roshini Jain, Lilly Chen, Thomas Brucke, Fernando Seijo, Esther Suarez San Martin, Claire Haegelen, Marc Verin, Mohammed Maarouf, Michael T Barbe, Steven Gill, Alan Whone, Mauro Porta, Domenico Servello, Lars Timmermann

Introduction

Deep Brain Stimulation (DBS) of the subthalamic nucleus (STN) for the management of motor symptoms of Parkinson's disease (PD) is typically performed under conscious sedation with awake evaluation during intraoperative physiologic testing (Machado et al., 2012). However, developments in surgical techniques now allow for subjects to be asleep during the procedure using general anesthesia. Previously reported long term outcomes of subjects who underwent STN-DBS under general anesthesia demonstrated postoperative safety and efficacy out to 1 year (Harries et al., 2012). In this study, we examine outcomes between subjects undergoing the STNDBS procedure who used general anesthesia versus those using conscious sedation with awake evaluation as part of the ongoing VANTAGE clinical trial.

Methods

VANTAGE is a prospective, multi-center, non-randomized, open-label interventional trial, sponsored by Boston Scientific Corporation. The trial assesses motor improvement in subjects with moderate-to-severe PD following bilateral STN-DBS. Assessments include motor scores such as Unified Parkinson's Disease Rating Scale (UPDRS) and quality of life measurements such as PDQ-39. Forty subjects were implanted bilaterally with the Vercise DBS System (Boston Scientific Corporation) at 6 European centers. Of these, 19 (47\%) underwent the DBS procedure under general anesthesia. $\underline{\text { Results }}$

Analysis of both study groups did not indicate a statistically significant difference in motor function outcomes. However, subjects utilizing general anesthesia were found not to display motor function outcomes that were necessarily clinically inferior to those consciously sedated with awake evaluation. Sample size and variation in baseline differences in selected subjects may have contributed to these final results, as a tendency for subjects undergoing general anesthesia to report an improvement in motor function similar to or better than those consciously sedated (versus baseline) was observed. Conclusion

No inferiority in clinical outcomes was observed in subjects that underwent general anesthesia versus those that were consciously sedated with awake evaluation. 
The Influence of Bilateral Subthalamic Nucleus Deep Brain Stimulation on Gastrointestinal and Urinary Dysfunction in Parkinson's Disease Patients

Julie G. Pilitsis, Heather Christine Smith, Claire Collison, Arun Chockalingam, Lucy Gee, Adolfo Ramirez-Zamora, Jennifer Durphy, Era Hanspal, Eric Molho, Anne Barba, Damian Shin

$\underline{\text { Introduction }}$

Gastrointestinal and bladder dysfunction is a prevalent symptom of Parkinson's disease (PD) that greatly impacts a patient's quality of life. How deep brain stimulation (DBS) affects these nonmotor symptoms of PD has not been explored.

\section{Methods}

We prospectively examined 15 PD patients to assess the role of bilateral subthalamic nucleus DBS (STN-DBS) treatment on gastrointestinal and bladder dysfunction. Patients completed six questionnaires pre-operatively and at 6 months post-operatively on symptoms including drooling, swallowing difficulty, and urinary complaints associated with PD.

\section{$\underline{\text { Results }}$}

Analysis of the surveys revealed two groups of subjects "responders" who had a significant improvement in the measured quality, in contrast to "nonresponders" who had worse or stable scores. Responders exhibited a significant improvement in swallowing difficulty $(\mathrm{p}<0.05)$ but not in drooling severity. Responders exhibited a significant improvement in quality of life based on urinary conditions ( $\mathrm{p}$ $=0.001)$ and voiding dysfunction $(\mathrm{p}=0.001)$ while also showing a trend towards improvement in urinary symptoms $(\mathrm{p}$ $=0.068$ ).

\section{Conclusion}

The results show that STN-DBS in PD patients has a significant positive effect on swallowing, voiding and quality of life related to urinary symptoms. How DBS affects these symptoms mechanistically requires further investigation.
Influence of Intracranial Air on Electrode Position and Clinical Outcomes Following Deep Brain Stimulation for Parkinson's Disease

Jessica N. Bentley, Zhe Guan, Karen Cummings, Kelvin Chou, Parag G. Patil

Introduction

Intracranial air (ICA) potentially influences the accuracy and efficacy of deep brain stimulation (DBS), but the influence of ICA on distal contact position is less well-studied. In this study, we analyzed several measures of electrode movement and clinical outcomes with ICA volume.

Methods

Prospective evaluation of 73 consecutive patients who underwent bilateral subthalamic nucleus DBS for Parkinson's disease. Immediate and follow-up CTs were analyzed for ICA, lead displacement, and targeting accuracy. Measures of clinical outcomes were also assessed.

$\underline{\text { Results }}$

For 146 leads, mean distal contact deviation was $1.2 \pm 0.7 \mathrm{~mm}$ and mean bowing of the proximal lead was $2.8 \pm 1.4 \mathrm{~mm}$. Mean targeting error was $2.0 \pm 1.2 \mathrm{~mm}$. Average ICA volume was $21.3 \pm 13.7 \mathrm{~cm}^{3}$, with significant correlation between ICA volume and proximal bowing of the lead $(\mathrm{p}=0.01)$, but not distal contact deviation or targeting error. MDS-UPDRS scores and LED were not correlated to ICA. ICA extremes also showed no difference in these parameters.

Conclusion

Though the proximal DBS leads bends in association with ICA, movement of the distal tip and targeting error do not correlate with ICA. This finding is maintained when analyzing extremes of ICA. Additionally, volume of ICA does not correlate with clinical outcomes. 


\section{Microelectrode Recording During DBS Surgery: A Comparison of Two Approaches}

Mark J. Nolt, Rajeev Polasani, Alison Monette, Michael Mercury, Cindy Zadikoff, Taras Masnyk, Michael Rezak, Joshua M. Rosenow

Introduction

Deep brain stimulation (DBS) has become the standard of care in treating cases of medically refractory movement disorders.

Awake microelectrode recording (MER) is often used to define the target for placement of the stimulating electrode.

We compare two approaches to MER utilized within our programs: single track and an array with four microelectrodes. We aim to show how they impact the track chosen for the stimulating electrode implant.

\section{Methods}

Direct targeting is performed based on imaging prior to surgery. The center track in the array approach is planned according to the target coordinates, as is the only track in the single track approach. More passes are done in both approaches as necessary. Final implant location is based on MER, somatotopy, efficacy and side effects. We analyzed 130 array implants and 81 single track implants.

\section{$\underline{\text { Results }}$}

In the array approach, implant tracks were as follows: center: 40 (30.77\%); anterior: $63(48.46 \%)$; posterior: $12(9.23 \%)$; lateral: $11(8.46 \%)$; medial: $0(0 \%)$, with $4(3.08 \%)$ outside the original array. For single track: center: 51 (62.96\%); anterior: $5(6.17 \%)$; posterior: 4 (4.94\%); lateral: 4 (4.94\%); medial: 13 $(16.05 \%)$, with $4(4.94 \%)$ placed in other tracks. The mean number of tracks was 4.12 in the array and 1.77 in the single track approach.

\section{Conclusion}

Our data show that the probability of implanting the stimulating electrode in the center track is greater in the single track approach, with more tracks done on average in the array approach. To determine the best approach for identifying implant location, outcomes data need to be analyzed. The array approach may identify the "best" location based on intraoperative data, while the location identified by the single track approach may prove just as effective postoperatively. Our data argues against the practice of asleep DBS where the center track (or planned target) is always selected, as both of our methods yield a high percentage of non-center track implants.

\section{Current Reimbursement Strategies May Discourage Routine Completion of Deep Brain Stimulation as a Single Stage Procedure Despite Potential Clinical and Financial Benefits}

\author{
John Richard Lamm, Craig van Horne
}

Introduction

Traditionally, deep brain stimulation (DBS) for Parkinson's disease has been completed as a two stage procedure. During one surgery, the pulse generator and lead extensions was implanted with the patient under general anesthesia. Implanting the stimulator leads in an awake patient during a separate procedure allowed for clinical observation to supplement earlier, less accurate stereotactic localization and microelectrode recording techniques. Recent technological advances allow surgeons to complete lead placement safely and effectively in patients under general anesthesia using intraoperative image guidance systems or with a combination of standard frame based localization and intraoperative microelectrode recording. Now that both stages of the procedure can be completed while patients are under general anesthesia, it is feasible for the entire DBS system to be implanted during a single, streamlined procedure.

Methods

Review of current literature regarding asleep DBS for Parkinson's Disease and Medicare reimbursement for DBS. $\underline{\text { Results }}$ Unfortunately, current Medicare reimbursement provides a disincentive to physicians and hospitals to complete DBS system implantation during a single surgery. Providers receive an average of $\$ 22,423.84$ to complete implantation of an entire system during a single procedure. However, providers receive $\$ 13,483.47$ and $\$ 26.152 .16$ for lead implantation and pulse generator implantation, respectively, when completed as two separate procedures for a total of $\$ 39,635.63$. Although the single stage operation would save on hospital costs, it is not enough to make up the difference of $\$ 17,211.79$. In fact, the difference in reimbursement would likely result in hospitals losing money every time a streamlined DBS implantation were completed.

Conclusion

Total DBS system implantation during a single surgery for patients under general anesthesia is now possible using new technology and techniques. Although it would be a more desirable option for patients and a more cost-effective option, reimbursement issues may prove to be a hindrance to surgeons wanting to offer this new treatment option. 


\section{Does Trajectory Matter? A Study Looking into the Relationship of Trajectory with Target Engagement and Error Accommodation}

\section{David Steel, Surajit Basu}

$\underline{\text { Introduction }}$

STN-DBS is now a life-altering treatment for advanced Parkinson's disease. The ideal target point within the STN is known. However, no emphasis on the impact of trajectory exists. The ellipsoid shape of the STN and the off-centre traditional target point means that variation in the electrode inclination should affect STN engagement. Understanding of this relationship could inform trajectory selection by improving STN engagements and margins of error.

\section{$\underline{\text { Methods }}$}

We simulated electrode placement at the clinical target through a set of trial trajectories. Twelve 3D-reconstructed STNs were created from MRI data of 6 patients. Each STN was approached through 56 simulated trajectories arranged in a grid covering a quadrant of skull around and in front of the coronal suture. A subset of 20 viable trajectories was reassessed for depth of engagement in each STN.

\section{$\underline{\text { Results }}$}

Group averages for each trajectory are presented as traffic light maps and as an overlaid skull mask illustrating recommended electrode entry sites. Trajectories under 30 degrees anterior to the bregma and between 10 to 30 degrees off the midline accommodated over 2.4 degrees of wobble. A mean engagement of $6 \mathrm{~mm}$ was possible in half of the subset. The longest engagements are on trajectories which saddle the coronal suture, extending to 40 degrees lateral. Microelectrode tracts of 14 additional STNs were collated using above protocol and engagement exceeded $5 \mathrm{~mm}$ in all central trajectories without capsular side effects, suggesting placement away from STN borders.

\section{Conclusion}

Trajectory selection influences engagement and flexibility to accommodate electrode wobble or brain shift. We recommend having the first trial 20 degrees anterior to the bregma, moving postero-laterally in successive trials to balance both error and engagement. When wider margins of error are beneficial (e.g. second side during bilateral procedures), trajectories nearer the coronal suture and around 25 degrees off the midline are advised.

\section{Stereotactic Error in Deep Brain Stimulation Electrode Placement is Related to Target}

Andrew Lin Ko, Aly Ibrahim, Kim J. Burchiel

Introduction

Deep Brain Stimulation (DBS) is a safe and effective therapy for Parkinson's disease, essential tremor and dystonia. Increasing use of intraoperative imaging allows near real-time assessment of stereotactic accuracy during implantation of intracranial leads. Frameless stereotactic placement is likewise gaining acceptance with accuracy comparable to frame-based techniques. This study is the largest assessment to date of stereotactic error using these techniques for the placement of DBS electrodes.

Methods

This is a retrospective study. Intraoperative CT imaging was reviewed in patients undergoing DBS placement for Parkinson's disease and essential tremor at Oregon Health and Sciences University. The AC/PC coordinates of the target electrode were compared to the operative to obtain Euclidean error and absolute error in the anterior-posterior, lateralmedial, and superior-inferior directions.

$\underline{\text { Results }}$

169 leads in 94 patients were examined. Targets were GPi $(\mathrm{n}=86), \operatorname{STN}(\mathrm{n}=31)$ and $\operatorname{Vim}(\mathrm{n}=52) ; 85$ were placed on the left and 84 on the right. Average Euclidean error was $1.63 \mathrm{~mm}$ (SD: 0.87). Target but not side showed significant effect on error magnitude, being higher for Vim (1.95 mm, SD: 0.88) than for GPi (1.44 mm, SD: 0.84), while STN (1.65 mm, SD: 0.88 ) did not differ from either Vim or GPi (ANOVA: target, $\mathrm{F}=6.15, \mathrm{p}=0.0027$; side, $\mathrm{F}=0.02, \mathrm{p}=0.893$ ). Electrodes targeting Vim were significantly more likely to deviate medially (Vim: $1.28 \mathrm{~mm}$; GPi: $0.49 \mathrm{~mm}$; STN: $1.06 \mathrm{~mm}$; Kruskal-Wallis: Chi-sq=16.15, $\mathrm{p}=0.00$ ). There was a correlation between a steeper coronal trajectory and larger magnitude of medial deviation when targeting Vim (rho=0.338, $\mathrm{p}=0.014)$.

Conclusion

This study shows a significant effect of target on the accuracy of electrode placement for DBS. Trajectories targeting Vim result in greater Euclidean error and greater medial deviation off target. These systematic deviations should be taken into account when using frameless stereotactic placement of DBS electrodes. 
Long-Term Quality of Life Outcomes Are Related to Patient Perception of Motor Symptoms Following Deep Brain Stimulation

Rebecca L. Achey, Erin Yamamoto, Bryan S. Lee, Sean J. Nagel, Andre Machado, Darlene Angela Lobel

Introduction

Deep brain stimulation (DBS) is a well-established therapy for treating motor symptoms of movement disorders. However, little is known about the impact of patient-perceived symptom severity on quality of life (QoL). Additionally, conflicting data exist regarding the effects of DBS on QoL measures, including depression. We analyzed the change in measures of tremor and QoL in DBS patients at our institution.

\section{Methods}

A retrospective chart review was performed including patients with Parkinson's disease and essential tremor. We analyzed data from pre-operative, post-operative and one-year followup visits including: the Patient-Health Questionnaire (PHQ-9, a standardized depression scale), UPDRS, and clinicianreported and self-reported tremor scores. Repeated measures ANOVA was used to examine changes in PHQ-9 and selfreported tremor from pre-operative to post-operative visits. Relationships between PHQ-9, UPDRS, clinician-reported and self-reported tremor scores were analyzed using linear regressions.

\section{Results}

Eighty-one patients who underwent unilateral or bilateral DBS at Cleveland Clinic from 2011-2013 completed PHQ-9 assessments. PHQ-9 scores significantly decreased from preoperative (8.3 \pm .66$)$ to post-operative $(6.7 \pm .62)$ and one-year follow-up visits $(5.8 \pm .54)[F(2,159.7)=8.89, p=.0002]$. Selfreported tremor scores, completed by 34 patients, significantly decreased from pre-operative (12.4 .79) to one-year follow-up $(6.6 \pm .82)[F(1.6,53.2)=23.9, p<.0001]$. Prior to DBS surgery, there was no relationship between PHQ-9 and self-reported tremor. However, at post-operative and one-year follow up visits, higher self-reported tremor scores were predictive of higher PHQ-9 scores $[(\beta=.28, \mathrm{p}=.043)$ and $(\beta=.45, \mathrm{p}=.007)$ respectively]. Interestingly, UPDRS and clinician-reported tremor scores were not predictive of PHQ-9 scores.

Conclusion

We observed long-term improvement in patient-perceived depression following DBS, accompanied by a reduction in self-reported tremor scores. More severe tremors were predictive of higher depression ratings post-operatively. These data suggest that patient-perceived DBS efficacy is critical to patient quality of life. Further investigation is warranted to understand the relationship between factors that influence how patients experience illness and outcomes after DBS.
A Novel Technique to Predict and Adjust for Microelectrode Recording Tract Error Prior to Dural Opening Using Intraoperative Computed Tomography

Ryan Benjamin Kochanski, Sepehr B. Sani

Introduction

Accurate lead placement is crucial in DBS surgery for both safety and therapeutic reasons. We describe a novel technique to predict the intended lead target location and adjust for predicted error prior to dural opening.

\section{$\underline{\text { Methods }}$}

Twenty patients underwent frame based (Elekta, Stockholm, Sweden) uni- or bilateral DBS (Medtronic model 3389, Minneapolis, MN) lead placements under local anesthesia using microelectrode recording (MER). After assembly of the stereotactic arc and headstage, placement of burr holes, and before dural opening, a guide tube was inserted through the headstage and set to rest on dura. Intraoperative CT imaging prior to dural opening was obtained and images were merged with preoperative CT and MRI studies. Using the FrameLink (Medtronic, Minneapolis, MN) planning software, a trajectory was created along the path of the guide tube. Once created, the trajectory was extrapolated to target depth and the coordinates recorded and compared to the initial plan.

Adjustments in the $\mathrm{X}$ and $\mathrm{Y}$ coordinates were made depending on the degree of the error in those respective planes. The guide tube and microelectrode were then inserted for MER. Once at target depth, the final coordinates were recorded and compared with corrected tract coordinates.

\section{Results}

Initial Euclidean error between planned trajectory and predicted trajectory as extrapolated by intraoperative preplacement CT (iPPCT) was $1.80 \pm 0.16$. iPPCT was used to make trajectory adjustments which improved Euclidean error between adjusted iPPCT and final lead placement to $0.93 \pm$ $0.09 \mathrm{~mm}$.

\section{Conclusion}

iPPCT can be a useful tool to accurately predict and adjust for error in MER tract and/or DBS lead placement prior to dural opening and entry into brain parenchyma. Confirming accuracy could potentially reduce the number of MER tract and/or DBS lead passes thus leading to safer outcomes and shorter operative times. 
Trigeminal Neuralgia without Vascular Compression Is Associated with a Smaller Posterior Fossa Volume: An MRI Volumetric Study

Ahmed M. Raslan, Gulsah Ozturk, Amr AlBakry, Abigail J. Rao, Jonathan Huang, Kim J. Burchiel

Introduction

Trigeminal neuralgia (TN) occurs and recurs in the absence of neurovascular compression (NVC) in a substantial portion of TN patients. While the contribution of NVC to the pathophysiology of TN is still a subject of debate, the cause of $\mathrm{TN}$ when there is no NVC is completely unknown. Here we examine neuro-anatomic differences between $\mathrm{TN}$ with and without NVC

\section{Methods}

A volumetric study was performed on TN patients who had undergone surgical treatment. Measurements were performed using reconstructed images of BFFE sequence magnetic resonance images. We studied 143 patients with TN1 (117 with vascular compression, and 26 without) and 25 patients with TN2 (24 with vascular compression and 1 without). Following measurements were obtained: cerebellopontine angle (CPA) volume, posterior fossa volume, pons volume, length of cisternal portion of trigeminal nerve, and transverse distance between the two porus trigemini. Student t-test was used to compare means between the two groups. The study was IRB-approved. Measurements were performed on OSIRIX.

\section{Results}

The posterior fossa volumes in cases of TN with NVC and TN without NVC were $144.5 \pm 18.24 \mathrm{~cm}^{3}$ and $136.174 \pm 18.23$ $\mathrm{cm}^{3}$, respectively $(\mathrm{p}=0.028)$. The CPA volumes were $1.41 \pm$ $0.98 \mathrm{~cm}^{3}$ and $1.148 \pm 0.657 \mathrm{~cm}^{3}$, respectively $(\mathrm{p}=0.154)$. The length of cisternal portion of trigeminal nerve were $8.38 \pm$ $2.17 \mathrm{~cm}$ and $8.77 \pm 1.39 \mathrm{~cm}$, respectively $(\mathrm{p}=0.38)$. The pons volumes were $2.49 \pm 0.63 \mathrm{~cm}^{3}$ and $2.49 \pm 0.18 \mathrm{~cm}^{3}$, respectively $(\mathrm{p}=0.98)$. The distance between the two porus trigemini were $3.26 \pm 0.23 \mathrm{~cm}$ and $3.29 \pm 0.1 \mathrm{~cm}$, respectively $(\mathrm{p}=0.51)$

\section{Conclusion}

The mean posterior fossa volume in TN patients without NVC is $8.3 \mathrm{~cm}^{3}(5.79 \%)$ smaller than the posterior fossa volume in TN patients with NVC. Smaller posterior fossa volume could contribute to development of TN. Larger and matched controlled comparisons are needed.

\section{Effects of Subthalamic Nucleus Deep Brain Stimulation on Motor Symptoms in Tremor Predominant Parkinson's Disease Patients}

Tamana Kaderi, Roy S. Hwang, Julia A. Prusik, Ellie Hobson, Adolfo Ramirez-Zamora, Eric Molho, Era Hanspal, Jennifer Durphy, Julie G. Pilitsis

\section{Introduction}

Subthalamic nucleus deep brain stimulation (STN DBS) is an effective treatment option for many symptoms of PD, including tremor. However, patients with asymmetric disease and tremor predominant PD may not have a UPDRS III OFF score $>30$, which is a criteria used by clinicians and payers. Here we compare outcomes from patients with tremor predominant PD with UPDRS III OFF scores $<30$ to patients with UPDRS III OFF $>30$ treated at our center.

Methods

We retrospectively review UPDRS III twelve month outcomes and medication usage patterns following STN DBS comparing 7 tremor predominant PD patients with UPDRS III $<30$ to 7 age- and gender-matched cohort with UPDRS III >30.

$\underline{\text { Results }}$

Both groups consisted of 2 females and 5 males with mean age of $65.0 \pm 7.3$ for UPDRS $<30$ and $61.9 \pm 12.5$ for UPDRS $>30$.

Average duration of disease before DBS is similar between the two groups (11.0 \pm 3.2 years v. $11.1 \pm 4.7$ years respectively). We noted a trend for patients with scores $<30$ to be on fewer LEDD meds preoperatively $(\mathrm{t}(\mathrm{df})=-2.052(12), \mathrm{p}=0.063)$.

Patients with scores $<30$ had a greater percentage improvement in UPDRS III OFF medication/ON stimulation score than patients with scores $>30(\mathrm{t}(\mathrm{df})=4.9(12), \mathrm{p}<0.001)$. Conclusion

We demonstrate that tremor predominant PD patients with a pre-operative UPDRS III OFF score $<30$ benefit significantly from STN DBS despite meeting an exclusion criteria for many studies and payers. 


\section{Pallidal Deep Brain Stimulation In the Treatment of Meige Syndrome}

Michael Roman Sobstyl, Miroslaw Zabek, Grazyna

Brzuszkiewicz-Kuzmicka, Tomasz Pasterski

$\underline{\text { Introduction }}$

Meige syndrome (MS) is characterized by blepharospasm, facial, oromandibular, and cervical dystonia. The medical treatment of this condition is challenging and unsuccessful over long time. Recent case reports and small clinical series showed that bilateral deep brain stimulation (DBS) of globus pallidus pars interna (GPi) improves dystonic features of MS validated by Burke-Fahn-Marsden Dystonia Rating Scale (BFMDRS) (1-5).

\section{Methods}

We report on our experience in using bilateral GPi DBS in 5 cases of MS. We present short-term (3 months) follow-up as well long-term (from 24 months to 48 months) results. Preoperative and postoperative BFMDRS assessments were performed on each patient. The postoperative BFMDRS scores was done when both stimulators were switched on and compared to baseline scores.

\section{$\underline{\text { Results }}$}

Bilateral GPi DBS reduced the BFMDRS total movement score by $75 \%$ at short-term follow-up, and by $87 \%$ at longterm follow-up when compared to baseline scores. The BFMDRS total disability score was reduced by $46 \%$ at shortterm follow-up, and by $56 \%$ at long-term follow-up when compared to baseline scores.

\section{Conclusion}

Our results showed that bilateral GPi DBS in MS is effective and safe, if conservative treatment options failed. The benefit is not only observed at short but also at long-term follow-up ranging from 24 to 48 months postoperatively.
Trigeminal Nerve Fractional Anisotropic Differences Between Radiosurgery Responders and Nonresponders

Justin Sharim, Wei-Lun Lo, Srinivas Chivukula, Tania B. Kaprealian, Nader Pouratian

Introduction

Trigeminal neuralgia (TN) is a neuropathic disorder characterized by high intensity electric shock-like attacks of unilateral pain. In medically refractory patients, neurosurgical interventions such as stereotactic radiosurgery (SRS) of the trigeminal nerve at or near the root entry zone (REZ) can relieve pain and effectively impact REZ microstructure. However, the mechanism of these interventions' influence on the trigeminal nerve and subsequent analgesic effect are not well understood.

Methods

Patients included in the study were any patients who underwent SRS for TN at Ronald Reagan UCLA Medical Center and who had diffusion tensor magnetic resonance imaging performed after treatment. Fractional anisotropy was extracted bilaterally from each trigeminal REZ. Pain reduction was measured in terms of Barrow Neurological Institute (BNI) scores at 6-month follow-up. Student's t-test was employed with a threshold of significance set at $\mathrm{p}<0.05$.

$\underline{\text { Results }}$

Twelve patients ( 3 males and 9 females) were included in this study. Seven of the twelve patients had good outcomes (BNI score 3a or less) following therapy. Patients with good responses to treatment had significantly larger fractional anisotropy values over the distribution of the trigeminal nerve of the affected side (mean \pm standard deviation: $0.29 \pm 0.075$ ) relative to patients with poor outcomes post-SRS $(0.16 \pm$ $0.092, \mathrm{p}=0.028$ ).

Conclusion

These results support a role of DTI in detecting subtleties of microstructural changes of the trigeminal nerve and suggest its potential use as an adjunct in assessing patient outcomes after treatment. The use of such metrics could permit a better means of prognosis and assessment of treatment response, bringing us closer to personalizing therapy through measures that are both objective and noninvasive. 


\section{Bilateral Anterior Internal Capsulotomy with Laser Ablation for Treatment of Obsessive Compulsive Disorder: Case Report}

\section{Ashley K. Ralston, Valentina Vasenina, Jon Grant, Peter C.} Warnke

\section{$\underline{\text { Introduction }}$}

We describe a novel use of laser ablation for lesioning of bilateral anterior internal capsules in the treatment of obsessive-compulsive disorder (OCD). To date, three major modalities have been used for the creation of lesions in patients with OCD - stereotactic radiosurgery (SRS), radiofrequency ablation (RF) and deep brain stimulation (DBS). Disadvantages to SRS and DBS include delayed time to therapeutic response, where RF ablations are performed without real time monitoring. Major advantages of MR guided laser ablation include the immediate effect, as well as the ability to monitor lesion size and design the lesion whilst we create it with real time MRI Thermography. This allows for more precise and reproducible lesions, which we hypothesize will correlate with improved outcomes.

\section{Methods}

We present one patient, suffering from refractory obsessivecompulsive disorder, who underwent MR-guided laser ablation of the anterior limb of bilateral internal capsules with Visualase Thermal Therapy system.

\section{Results}

She experienced no complications and a significant improvement in her OCD symptoms. Her pre-operative Yale Brown Obsessive Compulsive (YBOC) score was 39, which improved to 28 by 36 hours after surgery.

\section{Conclusion}

To the best of our knowledge, we present the first case of anterior internal capsulotomy with laser ablation for the treatment of OCD. Continued follow up, as well as further studies with larger cohorts, will be needed for validation of efficacy and safety.

\section{Industrial Lean Process Utility in Functional Neurosurgery: The Case of Microvascular Decompression}

Abigail J. Rao, Carli Bullis, Katherine Holste, Kim J. Burchiel, Ahmed M. Raslan

Introduction

Attending surgeons have concurrent obligations to deliver high quality health care and train residents. In modern healthcare, lean principles are increasingly being applied to the processes preceding and following surgery. However, surgeons have limited data regarding variability and waste during a given operation. We measured this variability, using a key functional neurosurgery procedure: microvascular decompression (MVD) for treatment of trigeminal neuralgia. We also correlate variability with surgeons' perceptions of risk and comfort, and aim to guide surgeons as they balance operative efficiency with training obligations.

Methods

We applied Plan, Do, Study, Act (PDSA) cycles to MVD. We created a standard workflow diagram, segmenting the basic components of the surgery. We timed these components for 15 operations, focusing on variation and wasted time.

Concurrently, we administered a survey regarding surgeons' perceived comfort and risk for each component. This was IRB approved. Statistical analysis was completed with R software. $\underline{\text { Results }}$

All surgical components were timed. Craniectomy had the highest mean duration and standard deviation, whereas the MVD itself had the lowest mean duration and standard deviation. Waste inventory showed a median wasted time of 3 mins (range 0-20 mins). There was a statistically significant relationship between increasing level of training and increasing perception of safety. We also show the relationship between assessment of danger and variability in duration for each component of the surgery.

Conclusion

Using a PDSA cycle and risk-matrix analysis, we identified components of high variability and quantified waste within a key neurosurgical procedure. We have also shown significant differences in perception of comfort and risk across training levels. Danger zones and highly variable components are targets for interventions to improve trainee comfort and operative efficiency. 


\section{How Does Torticollis Originating from the 11th Nerve Relate to Dystonia, and How Does This Affect Treatment? Our Experience with 32 Patients}

Franco Caputi, Guglielmo Cacciotti, Maria Pia Tonelli, Donatella Pantoli, Ettore Di Scipio, Luciano Mastronardi

\section{Introduction}

Torticollis is defined by official nomenclature as Cervical Dystonia (CD) and is related to a dysfunction of the basal ganglia. Despite contemporary authors favoring treatment by DBS, surgical approaches targeting the 11th nerve and its rami also yield positive results. This form of treatment is based on previous surgical experience and from insights gained from studies on vascular conflicts. Many patients have been operated focusing on the 11 th nerve. If we consider peripheral denervation alone, more than 2000 have been treated during the last thirty years. Our experience suggests that torticollis of 11 th nerve origin represents a special entity which benefits from selective intradural rhizotomy and microvascular decompression on the affected side.

\section{$\underline{\text { Methods }}$}

Patients were selected if they had a preeminent involvement of a SCM on one side. Clinical observation and EMG recording had to confirm a hyperactivity on this SCM with lack of inhibition when activating the controlateral SCM. We studied by MRI the intradural transition of vertebral artery (VA) and possible conflict with the 11th nerve, especially the McKenzie branch, or the 1 st cervical root.

\section{Results}

We always found a vascular conflict between the VA and the 11th nerve, in 13 cases with the McKenzie branch. Significant changes were obtained with regards to deformities, disabilities and pain score on Toronto Western Spasmodic Torticollis Rating Scale (TWSTRS) scale. The range of motion widened and patients regained the ability to turn against the previously affected SCM (ANOVA, P < 0.01). Results were documented by intraoperative recording in relation to preoperative clinical state and outcome.

Conclusion

Our observation confirms that there are cases of CD due to 11th nerve damage and that surgery based on this premise was successful. If other observers confirm these findings, we should consider a different nomenclature to guide surgery.
Use of the Putamen as Surrogate Anatomical Marker for the GPi in DBS Surgery

Dali Yin, John Thompson, Steven Ojemann, Aviva Abosch

Introduction

The success of deep brain stimulation of the internal segment of globus pallidum (GPi DBS) depends on accurately placing the electrode into GPi motor territory. Direct targeting can be difficult as GPi laminar borders are not always clearly identifiable on MRI. Here, we report a method for using the putamen (PUT) as a surrogate anatomical marker to identify $\mathrm{GPi}$, when tissue contrast is inadequate for direct visualization of the GPi DBS target.

Methods

Five patients with Parkinson's disease were included in this retrospective study. PUT-based GPi targeting was developed using FGATIR MR sequences as follows. The line connecting anterior (AC) and posterior commissure (PC) was drawn, followed by a line (A) through the anterior pole of PUT and parallel to the AC-PC line. The axial length of PUT was then divided into anterior, middle and posterior thirds. A second line (B) was drawn through the border between middle and posterior thirds of PUT. The intersection of A and B corresponded to the optimal location for DBS contact 0 in GPi, based on literature. GPi targeting was compared using PUT-based method vs. consensus coordinate-based indirect targeting. Stereotactic target coordinates were obtained and analyzed.

Results

PUT and GPi in all cases were visualized clearly on FGATIR. GPi borders were not identified on $\mathrm{T} 2$, but were localized correctly in all cases using PUT-based method on T2, merged onto FGATIR. Significant differences were noted in $\mathrm{Y}$ and $\mathrm{Z}$ target coordinates between PUT-based method and indirect targeting $(\mathrm{p}=0.02$ and 0.02$)$, but not $\mathrm{X}$ (mean differences for $\mathrm{X}, \mathrm{Y}$ and $\mathrm{Z}: 1.5,3.3$ and $6.0 \mathrm{~mm}$ ).

Conclusion

PUT can be used as a MRI marker for targeting GPi when GPi is not clearly visualized. Further study is planned to correlate PUT-based GPi targeting with microelectrode recording and clinical outcome. 


\section{Dorsal Percutaneous Chemo-Sympathectomy for Palmar Hyperhidrosis: A Case Presentation and Surgical Technique}

\section{Efkan Mustafa Colpan}

Introduction

Palmar Hyperhidrosis (PHH) is a functional disorder that negatively effects people both socially and psychologically. Cervical sympathectomy has been reported effective long term treatment for PHH. Author presents a successfully treated PHH case by the Dorsal Percutaneous Chemo-sympathectomy (DCS), an underutilized minimally invasive technique.

\section{$\underline{\text { Methods }}$}

A 36 years old woman presented with bilateral PHH. Patient did try medical management of PHH but failed. Patient developed social hesitancy and troubles in her job related to bilateral PHH. Patient underwent staged bilateral needle DCS using pure alcohol injection for PHH. Procedures were performed under local anesthesia in outpatient setting. Patient was positioned prone and percutaneous needle placements for alcohol cervical DCS were performed under fluoroscopy guidance.

\section{$\underline{\text { Results }}$}

Patient's right sided PHH completely resolved immediately after the procedure and the procedure was not complicated. Left sided DCS provided 30\% reduction of PHH. Left sided DCS procedure was also complicated by minor Horner's syndrome. Eventually, left sided Horner's symptoms resolved in five months. Unfortunately, left PHH recurred nine months after the first procedure. Left sided DCS was repeated. Second left sided DCS procedure was not complicated and provided 90\% reduction of $\mathrm{PHH}$. Complete resolution of right $\mathrm{PHH}$ and 90\% improvement in left PHH were observed during 16 months follow-up evaluation.

\section{Conclusion}

DCS is a percutaneous minimally invasive outpatient needle procedure. It is easily repeatable as necessary. DCS provides reasonable immediate and long-term treatment effects for PHH.

\section{Pericranial Flap Over the Hardware as the Enhancement for the Bur Hole Incision Closure in Deep Brain Stimulation (DBS) Surgery}

Viktoras Palys, Kathryn L. Holloway

Introduction

A frontal bur hole provides the traditional cranial access for DBS electrode lead placement. The Medtronic Stimloc is the most commonly used device to secure the lead. This creates a lump under the scalp which is unsightly in balding men as the scalp "shrink wraps" to the hardware. In addition, the risk of hardware erosion (1.24-9.65\% reported in literature) and introduction of bacteria to the hardware from a "spitting" stitch may all be related to the lack of protective layer between the scalp skin and DBS hardware. Although the usage of collagen matrix allografts helps with these issues, allografts tend to induce inflammatory response with weeping wounds. We report on a method to utilize the native vascularized tissue to provide protection of the DBS implant and improved cosmesis.

Methods

While making a straight scalp incision for the bur hole, we preserve the pericranium. A " $\mathrm{U}$ " shaped incision is then made in the pericranium which is reflected anteriorly. At the end of surgery, the DBS electrode lead is tunneled to the connector site incision and the residual lead is then coiled around the Stimloc. The pericranial flap is stretched to cover the hardware and is secured around its perimeter with cranial plating screws. The scalp incision is then permanently closed with 4-0 Vicryl interrupted galeal sutures and metal skin staples.

Results

Since December 2014, with more than 100 implanted DBS electrode leads, we have not observed any instance of DBS hardware erosion or "spitting" stitches leading to the explantation of the hardware. Additionally, the incision closures have been simpler and less costly while the cosmetic results have been better than that achieved with allografts. Conclusion

Vascularized tissue autografts have been utilized widely in tissue reconstructive surgeries. Our experience with the application of this technique to DBS surgery has demonstrated advantages over allografts. 


\section{Deep Brain Stimulation of the Internal Globus Pallidus for Disabling Haloperidol-Induced Tardive Dystonia. Report of Three Cases}

Michael Roman Sobstyl, Miroslaw Zabek, Grazyna

Brzuszkiewicz-Kuzmicka, Tomasz Pasterski, Artur Zaczynski

Introduction

Tardive dystonia (TD) represents a side effect of prolonged intake of neuroleptic drugs. TD can be a disabling movement disorder persisting despite available medical treatment. Deep brain stimulation (DBS) has been reported successful in this condition although the number of treated patients with TD is still limited to small clinical studies or case reports. In this study, we present results of bilateral globus pallidus internus (GPi) stimulation in 3 additional TD cases.

Methods

The formal assessment included the Burke-Fahn-Marsden Dystonia Rating Scale (BFMDRS). The preoperative and postoperative functional and motor parts of BFMDRS were compared in each patient.

$\underline{\text { Results }}$

Three patients underwent successful bilateral GPi DBS for TD. The postoperative BFMDRS motor score improved by mean of $71 \%$ at the last follow-up in each patient. There were no surgical or hardware-related complications over follow-up period.

Conclusion

Our experience indicates that bilateral GPi DBS can be an effective treatment for disabling TD. The response of TD to bilateral GPi DBS is very rapid and occurs within days after the procedure.

\section{Disease Stabilization of DYT1-Positive Primary Generalized Dystonia with DBS of the Globus Pallidus Interna: A 15-Year Follow-Up}

Sravanthi Koduri, Raj Kiran Nangunoori, Cindy Angle, Donald M. Whiting

Introduction

Primary generalized dystonia (PGD) is a genetic form of dystonia that frequently displays resistance to pharmacotherapy and progresses within the first few years after onset. Deep brain stimulation (DBS) has been used successfully in the treatment of refractory dystonia, specifically DBS of the Globus Pallidus pars interna (GPi) for patients with DYT1-positive PGD. Long-term follow-up of the safety and efficacy falls short of the longevity seen in other diseases treated with DBS.

Methods

Case report and long term follow-up of continual GPi stimulation in a patient with DYT1-positive PGD.

$\underline{\text { Results }}$

A male patient presented for neurosurgical evaluation with scapular winging, hand contractures, and violent truncal spasms which were debilitating to the point where he was bedridden. After failing conservative therapy, the patient was implanted with bilateral GPi-DBS at the age of 18. DBS parameter adjustments were made primarily within the first three years after implantation, with nominal changes in the ensuing years. Initial settings were contact of 3+0-, amplitude of $4.9 \mathrm{~V}$, frequency of $185 \mathrm{~Hz}$, and pulse width of $270 \mu \mathrm{sec}$ on the left and 3+0-, $2.8 \mathrm{~V}, 185 \mathrm{~Hz}$, and $120 \mu \mathrm{sec}$ on the right. Current settings are $3+2+1-, 5.2 \mathrm{~V}, 130 \mathrm{~Hz}, 330 \mu \mathrm{sec}$ on the left and 3+0-, $3.5 \mathrm{~V}, 185 \mathrm{~Hz}$, and $180 \mu \mathrm{sec}$ on the right and have been relatively unchanged in the past four years. Unified Dystonia Rating Scale (UDRS) scores reveal a significant decrease in dystonic symptoms.

Conclusion

While prior reports have shown that GPi-DBS is effective for dystonia, ours is the first with 15 years of long-term follow up showing disease stabilization, suggesting that stimulation is not only efficacious but prevents disease progression. This report reaffirms previous reports that recommend early surgical intervention prior to the onset of permanent musculoskeletal deficits. 
Educational and Career Instruction for Students Involved in Neurosurgical Research

Stephanie A. Owusu, Olivia Marola, Julia Prusik, Julie G. Pilitsis

Introduction

Early exposure to medical specialties and mentorship are key components of determining which residencies/fellowships students pursue. Involvement in functional neurosurgical research is one means of providing that exposure and mentorship. We evaluate our experience over 8 years with over 40 students.

Methods

We review the students that have participated in our functional neurosurgery research program, their time commitments, deliverables, and academic progress.

Results

We have had a total of 39 students rotate from January 2008 to August 2015, excluding neurosurgical residents and postdoctoral fellows. These were comprised of 2 high school, 7 undergraduate, 29 medical, and one MD/PhD student. High school and first year undergraduate students focused on database entry, proofreading, and review articles/presentations. Second year undergraduate and medical students were assigned projects to oversee from IRB/IACUC approval to completion. 18 medical and $\mathrm{MD} / \mathrm{PhD}$ students out of 29 have presented at at least 1 national meeting. 11 of those students have had their research presented at 3 or more national meetings. 20 of 29 of those same students have published a peer-reviewed paper. Approximately 10 hours a week are spent on mentorship with the amount of time commensurate with the skill set of the student and the type of project. Four of 11 graduates have gone on to neurosurgical residences with an additional 4 students embarking on a related specialty; neurology, neurooncology, research or orthopedics. To aid others, we have created a handbook to provide general background information for students working with our team to streamline the process of guiding students through research.

Conclusion

Mentoring students through a research project provides exposure to neurosurgery while cultivating student skills in fundamental concepts of research. Providing early mentorship opportunities to students will help guide them towards making informed career discussions.
Intracranial Magnetic Resonance Guided Laser Induced Thermal Therapy (MRgLITT): Single Center Experience in Patients with Epilepsy

Purvee D. Patel, Shabbar F. Danish

$\underline{\text { Introduction }}$

Magnetic Resonance Guided Laser Induced Thermal Therapy

(MRgLITT) has been progressing to successfully treat a large and expanding number of neurosurgical conditions including pathologies such as epilepsy. We report our single-center experience in 20 patients with epilepsy using the Visualase Thermal Therapy System.

\section{Methods}

A retrospective analysis was performed of all patients with epilepsy undergoing MRgLITT from 2011 to 2015. Laser catheters were stereotactically placed and ablation was performed in the MRI suite. Demographics, operative parameters, and hospital stay were recorded. Analysis also included review for any complications or readmissions following the procedure.

\section{$\underline{\text { Results }}$}

21 laser catheters were placed in 20 patients requiring intervention for epilepsy after other means of treatment had been exhausted. $100 \%$ of patients completed the procedure. Total procedural time was $150 \pm 25.8 \mathrm{~min}$ and laser on-time was $6.1 \pm 3.2 \mathrm{~min}$. Average ICU and hospital stay were 0.7 and 1.1 days, respectively, and the median stay for both ICU and hospital was 1 day. All patients were discharged home following procedure, none requiring discharge to rehabilitation facilities. There was a $0 \%$ complication rate. The 30 -day readmission rate was $0 \%$.

$\underline{\text { Conclusion }}$

MRgLITT is a minimally invasive, safe procedure that can be used for the treatment of epilepsy. Our single center experience yielded no complications or readmissions secondary to the procedure and most patients were stable for discharge within 24 hours. MRgLITT's therapeutic role in epilepsy will require further, more extensive studies. 
Episodic Hemi-Laryngopharyngeal Spasm: A Novel Cranial Neuropathy Treatable Surgically

Zurab Ivanishvili, Peter A. Gooderham, Murray Morrison, Christopher Honey

$\underline{\text { Introduction }}$

We describe a novel cranial neuropathy manifesting with lifethreatening episodic hemi-laryngopharyngeal spasm (HELPS).

Methods

We studied a case of a 50-year-old woman presenting with a four-year history of intermittent throat contractions, escalating to life-threatening respiratory distress. We provide the details of her diagnostic workup and the treatment strategies, including Botulinum toxin and surgery.

\section{$\underline{\text { Results }}$}

Botulinum toxin injections into her right pharyngeal muscles and vocal cord reduced the severity of her spasms, but the episodes continued to occur. MRI demonstrated a possible neurovascular conflict involving the cranial nerve IX-X complex and the posterior inferior cerebellar artery. Microvascular decompression (MVD) of the upper rootlets of the vagal nerve was performed, which eliminated her HELPS without complication. She remains free of spasmodic episodes at 1 year follow up.

Conclusion

We propose a mechanism of episodic hemi-laryngopharyngeal spasm implicating isolated involvement of the upper motor rootlets of the vagus nerve. HELPS syndrome can be treated with MVD.

\section{An Analysis of Multiple Novel Risk Factors for Deep Brain Stimulator Infections}

Elena Nehrbass, Nicholas James Brandmeir, James McInerney

\section{Introduction}

Deep Brain Stimulation (DBS) has become the most widely used treatment modality for medically refractory movement disorders. Although uncommon, infection and skin erosion are a particular problem because they require further surgery to treat, can jeopardize re-implantation, and cause significant patient discomfort. DBS infections can also progress to life threatening intracerebral infection. Many strategies to reduce DBS infections focus on preserving skin health and integrity. No study has yet looked at an objective association between scalp health and later infection. The goal of this study was to examine several patient factors for association with DBS infection.

Methods

This study was a review of a prospectively gathered singlecenter, single-surgeon database of DBS patients. All patients who experienced an infection were identified. The patient was considered to have had an infection if they required antibiotic treatment post-op for wound problems, reoperation for wound dehiscence or erosion, explantation because of infection, or suffered an intracerebral infection. Predictor variables considered were scalp thickness, routine intraoperative cultures, operative time, diagnosis, and age.

$\underline{\text { Results }}$

None of the independent variables examined were significantly associated with DBS infections.

Conclusion

DBS infections are independent of all of the predictor variables analyzed. New studies must examine new variables that are not routinely gathered in this population such as years with a diagnosis, functional status, tobacco use, diabetes, and nutritional factors. Future studies looking at DBS infections should be done as part of a large, prospectively designed, multicenter patient registry to increase the likelihood of discovering a predictor of infection. DBS infections may be a largely stochastic occurrence. 


\section{Subcortical Targeting in Non-human Primates Using Magnetic Resonance Image Guidance}

Jessica N. Bentley, Siri Sahib Singh Khalsa, Michael

Kobylarek, Kevin Showen Chen, Karen Schroeder, Derek Tat, Cindy A. Chestek, Parag G. Patil

Introduction

Cortical brain-machine interface (BMI) devices and subcortical single unit recordings have provided a great deal of insight into brain networks. However, to achieve greater understanding of complex networks, multi-unit recordings are needed from interconnected, deep areas of the brain. Since many studies will be performed in non-human primates (NHP), a reliable method of image-based targeting is needed to deliver devices with submillimeter precision. Here, we devised a precise and easily adaptable method of delivery to small, deep areas of the primate brain.

\section{Methods}

We performed magnetic resonance imaging (MRI) on 10 rhesus macaques ( $\mathrm{n}=7$ male, 3 female; $4.7-11.5 \mathrm{~kg}$ ) to determine optimal experimental design and ransformation algorithm for MRI based targeting. We also built a custom graphical user interface (GUI) to assist in planning, visualization, and analysis of optimal centroid target locations. The protocol was then implemented in vivo for hippocampal catheter delivery in a rhesus macaque.

\section{Results}

Fiducial markers (1.5 x $4 \mathrm{~mm}$ titanium screws) were implanted via stab incisions in lightly sedated animals and were easily localized on MRI. Image-space coordinates were obtained and the animal was positioned into a stereotactic headframe to derive surgical coordinates. The GUI coregistered and transformed coordinate points, and also enabled analysis of fiducial registration error (FRE) in real-time. In addition, it provided estimates of the expected target registration error (TRE) based on fiducial configuration. An in vivo experiment verified catheter delivery to hippocampus using the described approach.

Conclusion

A reliable, precise method of image-based targeting in NHP brain is achievable using easily obtained materials, without the need for expensive equipment. Additionally, this novel method is not timelocked, enabling more practical use of operating rooms and preoperative planning. This method will allow more extensive studies of multiple, deep, subcortical areas of interest for BMI device design.

\section{Stereotactic Frame Registration with Intraoperative CT, Comparison with Standard CT}

Joshua M. Rosenow, Andreas Haukas, Chad Jacobs, Mark J. Nolt

Introduction

Stereotactic frame registration is often carried out using a standard CT scan obtained in the radiology department. With the availability of better intraoperative CT scanners the opportunity for streamlining the workflow for these cases now exists. We sought to determine if the AIRO scanner (Brainlab) was capable of performing scans adequate for frame registration and how the target coordinates provided by this registration compared with those obtained from a standard CT scanner.

Methods

Ten consecutive patients undergoing DBS surgery underwent standard CT scans to register the Leksell frame for DBS surgery. They also underwent low dose scans with the AIRO scanner. These scans were uploaded to Brainlab iPlan Stereotaxy 3.0 software. The frames were registered from these scans and the scans were merged with preoperative MRI. Commissural coordinates were obtained, as well as frame settings ( $\mathrm{x}, \mathrm{y}, \mathrm{z}$, arc and ring angles).

$\underline{\text { Results }}$

The frame was successfully registered in all cases, although more adjustment of window and level were required for the AIRO scans. All coordinates compared favorably. No pair of coordinates differed by more than $0.84 \mathrm{~mm}$ between scan registrations. The mean difference across all coordinates was $0.18 \mathrm{~mm}$. The largest mean difference between coordinate pairs was $0.21 \mathrm{~mm}$ for both the target $\mathrm{Y}$ and $\mathrm{Z}$ coordinates. There was no significant difference in the variation between scans for the AC, PC, or target.

Conclusion

The AIRO scanner is capable to register the Leksell frame and provide coordinates comparable to those obtained with a standard CT scan. Abandoning the trip to radiology may save time and effort as part of stereotactic case workflow. This may also reduce total patient radiation exposure by using the AIRO low dose sinus scan mode. 
Impact of Trajectory Planning with Susceptibility Weighted Imaging for Intracranial Electrode Implantation

Guilherme Barros, Michael J. Lang, Ashwini Dayal Sharan, Chengyuan $\mathrm{Wu}$

$\underline{\text { Introduction }}$

Recently, susceptibility weighted imaging (SWI) has been used along with traditional T1-weighted gadolinium-enhanced (T1-Gd) MRI for trajectory planning during deep brain stimulation (DBS) and stereoelectroencephalograpy (SEEG) procedures. This study was performed to determine the effect of SWI versus T1-Gd vessel resolution on hemorrhagic complications during electrode implantation.

\section{Methods}

Fourteen patients undergoing DBS or SEEG electrode placement were implanted with a total of 114 electrodes. All patients underwent 3D CT and MR imaging post-operatively, and all trajectories were planned without use of SWI sequences. Post-operative CT images were co-registered to pre-operatively acquired T1-Gd and SWI sequences. Images were analyzed for presence of electrodes intersecting or passing within $1 \mathrm{~mm}$ of cerebral vessels on both T1-Gd and SWI sequences.

\section{$\underline{\text { Results }}$}

No patients had procedure-related intracerebral hemorrhage on post-operative MRI. Sixty unique instances of electrodes intercepting cerebral blood vessels were identified on SWI sequences (rate per electrode $52.6 \%$, and average 4.3 interceptions per patient), of which 34 were superficial and 26 were deep in location. Eighteen interceptions were identified on T1-Gd (rate per electrode 15.8\%, and average 1.3 interceptions per patient). There was a statistically significant difference in mean diameter of vessel intercepted, measuring $1.50 \pm 0.5 \mathrm{~mm}$ on SWI, and $2.12 \pm 0.7 \mathrm{~mm}$ on T1-Gd images $(\mathrm{p}=0.00024)$. Addition of electrodes passing within $1 \mathrm{~mm}$ of cerebral vessels resulted in total 104 total instances ("interception plus near-miss") on SWI (91.2\% per electrode, 7.4 per patient) and 23 instances on T1-Gd (20.2\% per electrode, 1.6 per patient).

Conclusion

Susceptibility weighted imaging is likely over-sensitive for electrode trajectory planning. There were no hemorrhages despite the high rate of electrode interception of or close proximity to cerebral vessels on SWI compared to T1-Gd imaging.

\section{Placement of Responsive Neurostimulation Depth Electrodes with Intra-operative MRI utilizing Real-Time Image-Guided Neuronavigation}

\author{
Jason L. Gerrard, Tim J. Goble, Dennis D. Spencer
}

Introduction

Accuracy of intracranial electrode placement is paramount for chronic neuromodulation. With approval of the Neuropace system, there is a significant increase in implanted electrodes targeting seizure onset zone rather than stereotactic coordinates. For example, the narrow amygdala-hippocampal complex, surrounding anatomy, and trajectory length require precision. Here we describe the first series of patients undergoing implantation of NeuroPace electrodes utilizing iMRI and ClearPoint system.

Methods

The IMRIS 3T iMRI and ClearPoint targeting system were utilized for three consecutive patients undergoing implantation of amygdala-hippocampal RNS electrodes. The target location was identified based on prior intracranial recordings of seizure onset. During the procedure, entry, target, and trajectory were defined by acquisition of initial iMRI. Following placement of ClearPoint SmartFrame, a second iMRI was obtained to reconfirm target and to account for intraoperative changes to target and trajectory. Following alignment of Clearpoint SmartFrame, a volume MRI was obtained to evaluate position of stylet/cannula. The RNS electrode was then placed through the cannula and a postoperative CT scan was obtained to confirm final placement. $\underline{\text { Results }}$

All four RNS electrodes were placed accurately and safely with a single brain insertion. There were no intraoperative or postoperative complications from electrode placement. For each trajectory, the radial, depth, and absolute error were calculated, along with target depth, based on the defined target versus the placement of MRI-visible stylet to target (mean \pm $\mathrm{SD}$ ). Radial error $=0.725 \pm 0.41 \mathrm{~mm}$, depth error $=1.075 \pm$ $0.38 \mathrm{~mm}$ deep, absolute error $=1.338 \pm 0.45 \mathrm{~mm}$, and target distance $93.23 \pm 3.65 \mathrm{~mm}$.

Conclusion

We report the first series of RNS electrodes placed utilizing iMRI and ClearPoint navigation. This method is feasible, safe and highly accurate. Furthermore, it allows for real-time knowledge of electrode placement, prior to the implantation of the RNS electrode without leaving the operating table. 


\section{International Tourette's Syndrome Deep Brain Stimulation Registry and Database}

Alon Y. Mogilner, Michael Pourfar, Wissam Deeb, Kyle Rizer, Justin Rossi, Richard Dowd, Michael S. Okun

Introduction

Emerging evidence from clinical studies suggests that DBS is effective in treating the symptoms of refractory Tourette Syndrome (TS). The data, gleaned from multiple case reports and small case series, has been notable for significant variation in target selection, outcome measures, and length of follow-up. The International TS DBS Registry and Database is a central data repository for the worldwide experience.

\section{Methods}

The International TS Registry and Database currently consists of 31 participating institutions in Australia, Europe, China, Japan, Canada and the USA. To date, 16/31 sites have submitted data, reflecting 160 individual patients. Our own single-center experience in 13 patients, included in the registry, was compared to the entire worldwide experience. $\underline{\text { Results }}$

The brain target structures utilized include 94 medial thalamus, 41 posteroventral GPi, 23 anteromedial GPi, and 2 nucleus accumbens region. The mean age of disease onset is 7.8 years, and average at surgery was 29.6 years. $18 \%$ reported a history of self-injurious behavior. Mean Yale Global Tic Severity Scale was 74.1 at baseline, and 37.8 at 24month follow-up. There were $80 / 160$ records $(50 \%)$ that contained information on specific adverse events. Devicerelated adverse events were noted in $15 \%$ of cases, the most common being device infection/erosion. The device explant rate was $17 \%$, with the reasons for explant including devicerelated complications, lack of clinical response, as well as spontaneous improvement in tics. In our single-center experience of 13 patients with a median f/u of 23 months, mean patient age at surgery was noted to be 10 years younger (19.9 YO), with a slightly higher baseline YGTSS (85), but with a similar reduction at latest follow-up (51\% reduction, 85->42).

Conclusion

Given the small number of DBS surgeries performed for TS, and variation in the field, more information will be necessary to plan next steps in this area. Though there are gaps in the data, encouraging collaboration and more standardization of data sets will drive the field and likely improve outcomes. Any individual or institution can join the collaborative effort.
Intrathecal baclofen therapy for GAD (+) Stiff Person Syndrome: A Case Series of 3 Patients

\author{
Lior Ungar, Takaomi Taira, Zion Zibly
}

Introduction

Stiff person syndrome is a rare disorder (the prevalence is estimated at about 1/1,000,000) of unclear etiology, characterized by progressive rigidity and stiffness. The stiffness primarily affects the truncal muscles and is superimposed by spasms, resulting in postural deformities. Stiff person syndrome is an autoimmune disorder and highly correlates with the presence of anti glutamic acid decarboxylase (GAD) antibodies. Baclofen and benzodiazepines are the primary treatments for SPS. Autoimmune suppressive treatments such as steroids, rituximab and plasma exchange have been also used in SPS patients; however, when the above treatments are inadequate, intrathecal baclofen may be used.

Methods

We reported 3 patients with GAD (+) SPS which presented severe refractory neurological manifestations of the disease and greatly benefited from intrathecal baclofen (ITB) therapy. $\underline{\text { Results }}$

A 34 year old man diagnosed with SPS presented with refractory progressive spasms of the lower trunk. Treated with implantation of programmable baclofen pump, the patient's spasm had decreased dramatically. The second patient was a 33 year old woman diagnosed with acute SPS developed to a life threatening disease manifested in progressive diaphragmatic spasm, leading to respiratory failure. While hospitalized at the intensive care unit, the patient underwent a promising intrathecal baclofen administration trial following an implantation of intrathecal baclofen pump. The diaphragm contractility was regained and the patient weaned off from mechanical ventilation soon after. The third patient was a 17 year old girl presented with truncal muscle spasm that was induced by voluntary movements, resulting in flexion of the arms and extension of the legs. Two months after the patient's admission, the patient demonstrated progressive breathing difficulties due to muscle spasm, and she had to be managed with mechanical ventilation. After an ITB pump implantation, the patient's condition rapidly improved, and after withdrawing from mechanical ventilation and a short period of rehabilitation, the patient had been discharged from the hospital fully functional, with a substantial reduction in her symptoms.

Conclusion

Intrathecal baclofen (ITB) therapy is a highly recommended therapy in the early stages of SPS. 


\section{Bilateral STN DBS Is Superior to Unilateral for Parkinsonian Gait under Cognitive Loading}

Gaurav Chenji, Melissa Wright, Kelvin Chou, Rachael D. Seidler, Parag G. Patil

$\underline{\text { Introduction }}$

Gait abnormalities are particularly challenging to subjects with Parkinson's disease (PD) due to increased fall risk. These risks may be increased under conditions of simultaneous cognitive loading. In fact, bilateral STN DBS has been shown to impair upper extremity function under cognitive loads. The aim of this study was to compare the effects of unilateral and bilateral STN DBS on gait with and without cognitive loading in advanced Parkinson's disease.

\section{$\underline{\text { Methods }}$}

Seventeen advanced PD subjects (ages 50 to 74 years) who had bilateral STN DBS surgery at least 6 months prior were studied, off medications. All data were collected while subjects were off their anti-parkinsonian medication for at least 12 hours. Quantitative gait information was collected with a pressure-sensor mat (GaitRite) during three stimulation states: left stimulation, right stimulation and bilateral stimulation. For each stimulation state, subjects performed three tasks: gait alone, gait while counting by $3 \mathrm{~s}$, and gait while naming alternate letters of the alphabet.

\section{Results}

As gait task complexity increased, there was a significant decline in performance for all stimulation states. Unilateral stimulation performance was comparable to bilateral stimulation performance for most gait parameters. However, bilateral stimulation surprisingly demonstrated improvement in step length and double leg support time, compared to unilateral stimulation, under conditions of cognitive loading. Conclusion

These results underscore the efficacy of bilateral subthalamic nucleus stimulation over unilateral stimulation on gait under dual-task conditions. Our findings suggest that differential advantages of bilateral and unilateral stimulation may occur for upper and lower extremity tasks under cognitive loads.

\section{Lessons Learned from 100 Consecutive Pediatric Deep Brain Stimulator Patients: The Cook Children's Hospital Experience}

\author{
John H. Honeycutt, Warren Marks, Fernando Acosta
}

Introduction

Since 2007 we have offered Deep Brain Stimulator (DBS) placement in select pediatric movement disorder patients at Cook Children's Hospital. Over time we have modified the surgical approach to help increase efficacy and minimize morbidity.

Methods

An Institutional Review Board (IRB) approved retrospective review was performed on our first 100 DBS patients. All patients enrolled for DBS surgery at Cook Children's Hospital undergo a rigorous consent process approved by our IRB in accordance with the current United States FDA Humanitarian Device Exemption status of this device for dystonia.

$\underline{\text { Results }}$

The average age at implantation was 13.8 years. Diagnosis was primary dystonia in 34 patients, secondary dystonia in 61, and tremor in 5. Implant site was globus pallidus internus (GPi) in 92, ventral intermediate nucleus (VIM) of thalamus in 4, subthalamic nucleus (STN) in 3, and one combo of GPi / STN. Complications were common with 12 infections in 9 patients, 8 strokes or hemorrhages in 8 patients, 15 electrode revisions (4 fractured, 11 repositioned) and 8 extension wire revisions due to malfunction / fractures. The first 80 patients were done awake with microelectrode recordings, but have transitioned to asleep surgery for the last 20 patients with no change in outcomes

Conclusion

Over the last 9 years, we have modified our surgical approach to minimize complications (more lateral trajectory to decrease strokes / hemorrhage, two stage placement to decrease infection, and asleep surgery to increase comfort). Although fraught with complications, pediatric DBS affords another viable treatment option that movement disorder teams can offer to these challenging patients. 
Long-Term Task and Dopamine Dependent Dynamics of Subthalamic Local Field Potentials in Parkinson's Disease

Sara J. Hanrahan, Joshua Nedrud, Bradley Davidson, Sierra Farris, Monique Giroux, Aaron Haug, Rajeev Kumar, Mohammad Mahoor, Anne Silverman, Jun Zhang, Adam Olding Hebb

\section{$\underline{\text { Introduction }}$}

Subthalamic Nucleus (STN) Local Field Potentials (LFP) are considered the most informative feedback signal for a closedloop deep brain stimulation (DBS) system. Traditionally, data collection of STN-LFP in humans has been restricted to the operating room during DBS implantation.

Methods

We use the Medtronic Activa PC+S system to record STNLFP in the clinic to determine the reliability of the STN-LFP over time and assess dynamics of the STN-LFP with behavior and dopaminergic medication. Six subjects were implanted with the PC+S system. Bilateral STN-LFP recordings were performed in the operating room and in the clinic at one, three, six, and twelve months after implant. Subjects were cued to perform voluntary behaviors including left and right hand movement, left and right arm movement, mouth movement, and speech.

\section{$\underline{\text { Results }}$}

We observed inter-subject variability that led us to focus on intra-subject analysis. STN-LFP movement related power changes in Beta $(13-30 \mathrm{~Hz})$ and Gamma $(40-70 \mathrm{~Hz})$ frequency bands were consistent across multiple recordings. The STNLFP recorded with the $\mathrm{PC}+\mathrm{S}$ demonstrated movementmodulated desynchronization of beta frequency and synchronization of gamma frequency oscillations. Medication did not diminish the magnitude of the beta frequency oscillatory desynchronization with movement. However, movement related gamma frequency band oscillatory synchronization was only observed in the medication on state. Conclusion

These findings demonstrate that the $\mathrm{PC}+\mathrm{S}$ system provides robust STN-LFP recordings in ambulatory patients, allowing for these signals to be recorded in settings that better represent the real-world in a variety of medication states. These results suggest oscillatory power in beta and gamma frequency bands of STN-LFP modulates with movement and medication state. Consistent with the literature, synchronization of gamma frequency oscillatory power was only observed in the medication on state, whereas desynchronization of beta frequency oscillations was readily observed in both the medication off and on state.

\section{Complication Rates and Length of Stay in Awake and Asleep Deep Brain Stimulation}

Tsinsue Chen, Zaman Mirzadeh, Kristina Chapple, Margaret Lambert, Francisco A. Ponce

Introduction

As the number of deep brain stimulation (DBS) procedures performed under general anesthesia ("asleep" DBS) increases, it is important to assess the rate of adverse events and inpatient length of stay for these patients compared with those undergoing traditional "awake" DBS without general anesthesia. There have been no previous studies evaluating total complication rates or inpatient lengths of stay for asleep DBS surgery.

$\underline{\text { Methods }}$

All patients undergoing asleep or awake DBS by a single surgeon between August 2011 and August 2014 were reviewed. Adverse events and inpatient length of stay were analyzed for 490 electrodes placed in 284 patients.

$\underline{\text { Results }}$ 126 patients (63.7\%) underwent asleep surgery, and 158 patients $(56.6 \%)$ underwent awake placement. The most frequent complication was postoperative mental status change (13 patients, $4.6 \%$ ), followed by hemorrhage (4 patients, $1.4 \%)$, seizure (4 patients, $1.4 \%)$, and hardware-related infection ( 3 patients, 1.1\%). Mean length of stay for all 284 patients was $1.18 \pm 1.22$ days (awake: $1.05 \pm 0.46$ days; asleep: $1.28 \pm 1.59$ days; $\mathrm{p}=0.12$ ). There were no significant differences in complications and length of stay between awake vs. asleep cohorts. Within the asleep cohort, a significantly higher proportion of patients with mental status change were greater than or equal to age 65 compared to those without mental status change $(\mathrm{p}=0.003)$.

Conclusion

Both awake and asleep DBS can be performed safely with low complication rates. We found no significant differences between the two technique cohorts when comparing adverse events and inpatient lengths of stay. 


\section{Deep Brain Stimulation of the Dentato-Rubro-Thalamic Tract: Outcomes of Direct Targeting for Tremor}

Albert J. Fenoy, Mya Schiess

$\underline{\text { Introduction }}$

New targets for Deep Brain Stimulation (DBS) for tremor control have recently gained popularity. Modulation of the cerebello-thalamo-cortical circuit via the dentato-rubrothalamic tract (DRTt) has been recognized as effective for tremor suppression. Our prospective observational study analyzed outcomes after using direct targeting of the DRTt. Methods

20 consecutively enrolled intention tremor patients obtained pre-operative 3T MRI with diffusion tensor (dTi) sequences. The DRTt was drawn for each individual patient on StealthViz dTi software (Medtronic) using the dentate nucleus as the seed region and the ipsilateral pre-central gyrus as the end region, which was then directly targeted during DBS surgery. Intraoperative testing confirmed successful tremor control. Post-operative analysis of electrode contact (EC) position as seen on CT relative to the DRTt was performed. The volume of tissue activated (VTA) was estimated using therapeutic EC parameters and an isotropic model. Post-operative clinical evaluations were performed at 6 months.

Results

The mean age of patients was 65.3 years; mean duration of tremor was 12.6 years. Most patients (95\%) had distal upper extremity tremor; 1 patient $(5 \%)$ had proximal bilateral upper extremity tremor. Head tremor was seen in 7/20 (35\%). Mean voltage for the $\mathrm{L}$ electrode $=3.35 \mathrm{~V} ; \mathrm{R}=2.6 \mathrm{~V}$. Mean distance from the center of the active EC to the DRTt was $0.90 \mathrm{~mm}$ on the $\mathrm{L}$, and $0.82 \mathrm{~mm}$ on the R. After calculating the estimated VTA for each therapeutic EC, only 1 (2.5\%) VTA fell outside the respective targeted DRTt, although the patient reported 90\% tremor improvement. Overall, there was $93 \%$ tremor control. All patients self-reported improved quality of life. Conclusion

Direct targeting of the DRTt is effective in tremor suppression. Accounting for both model and software limitations, electrode placement directly affecting these fiber tracts reproducibly produced tremor control; it is our preferred target.

\section{Motor-Related Neuronal Activity in the Cerebellar Thalamus}

David J. Segar, Shane Lee, Stephanie R. Jones, Wael Asaad

Introduction

The ventral intermediate nucleus of the thalamus (VIM), a key link between the cerebellum and the sensorimotor cortex, has long been a neuromodulatory target for pathologic tremor. Stimulation of VIM can dramatically reduce tremor in these patients; however, the underlying mechanisms by which VIM modulates motor output are poorly understood.

Methods

We obtained VIM single-neuron recordings from 6 essential tremor patients during DBS electrode implant surgery.

Waveforms surpassing a voltage threshold were sampled at 40 $\mathrm{kHz}$ and sorted using principal components. During recording, patients performed an 8-direction "center-out" task using a joystick to move a cursor to a target positioned around a circle. On some trials, the target jumped to a new location just after movement onset. Statistical significance for all findings was determined by comparing empirical values to a null distribution generated using non-parametric bootstrap methods.

$\underline{\text { Results }}$

Each neuron was tested for task-related responsiveness by comparing baseline firing rates to firing rates cued to specific task-related events. Of 59 neurons recorded, $\sim 50 \%$ responded to at least one task-related visual cue or motor event. Multiple subsets of responses were categorized, including responses selective for visual cues or motor events. Other neurons demonstrated similar responses to both visual and motor events, suggesting that these neurons may be involved in visual motor transformation. Approximately $20 \%$ of all neurons exhibited classical directional tuning. A small subgroup of neurons responded significantly more to the appearance of a "jumped" target and the associated movement correction. These neurons may be directly related to the transmission of a motor error correction signal.

Conclusion

VIM neurons were actively engaged by this joystick movement task, and many were tuned to the direction of movement. Several also selectively responded to corrections of movement, supporting a role for the VIM in conveying alterations in motor plans from the cerebellum to the cortex. 


\section{Short-Time Prediction of Motor Performance Using Local Field Potential in Parkinson's Disease}

Minkyu Ahn, Shane Lee, Julie Guerin, David J. Segar, Tina

Sankhla, Wael Asaad

Introduction

Motor deficit in Parkinson's Disease is typically assessed by subjective measures such as the Unified Parkinson's Disease Rating Scale (UPDRS) that measure un-naturalistic and discontinuous movements, and may not generalize to continuous limb movements. In this study, we present a simple joystick task that overcomes these limitations by monitoring motor errors in continuous, naturalistic movements with second-timescale precision. We also investigated local field potentials (LFP) in the subthalamic nucleus (STN) to investigate the relationship between behavioral performance and neural states.

Methods

The task we designed required subjects to follow a target that moves in one of several invisible patterns as closely as possible with a cursor controlled by a joystick. Each trial lasts 10 to 20 seconds depending on the pattern. From four patients, we collected the $\mathrm{X}$ and $\mathrm{Y}$ positions from joystick trajectories and LFPs in the STN during awake Deep Brain Stimulation (DBS) surgery.

Results

Two performance metrics were identified: tremor magnitude (TM), which quantifies physiological tremor amplitude, and vector error (VE), which quantifies how well a patient tracks the moving target by measuring the vector error between the target and cursor movements. Motor behavior was analyzed in a sliding 2-second epoch. We found epochs with higher VE or TM were associated with broadly elevated LFP power. Using frequency bands from $2-400 \mathrm{~Hz}$ with a $3 \mathrm{~Hz}$ step as features, machine learning algorithm (Support Vector Machine) based on VE or TM was able to predict behavioral motor performance with $78.6 \%$ and $82.9 \%$ accuracy, respectively. Conclusion

These results demonstrate that motor performance can be predicted on a short time scale using broadband LFP power. This approach could be useful for implementing an adaptive, closed-loop DBS system.
Limb and Laterality Somatotopies for Unilateral STN DBS

Mahesh B. Shenai, David Remer, Alaa Alhussein, Harrison Walker, Barton L. Guthrie

Introduction

Active contact location is a key driver for clinical success in STN DBS. This study aims to generate somatotopies of limb effect (upper extremity [UE] vs. lower extremity [LE]) and laterality (contralateral [CL] vs. ipsilateral [IL]), based upon categorization of the UPDRS Part III scoring symptom, and its spatial interpolation.

$\underline{\text { Methods }}$

PD patients receiving single-sided STN DBS, with 3 month post-operative follow-up were identified. Implantations were performed using a $\mathrm{CRW}$ frame-based technique, using local anesthesia, and microelectrode recordings (MER). After initial targeting, MERs and macrostimulation guided intraoperative trajectory adjustments. Patients were assessed pre-operatively and post-operatively (UPDRS Part III). The subcomponents of this scale were aggregated into quantitative UE/LE and CL/IL scores, and a percent reduction. Active contact locations were derived from postoperative MRI. An ordinary kriging method generated spatial topographies for each subcategory, at axial slabs $(z=-3.5$ and $-4.5 \mathrm{~mm})$. Fidelity of interpolation was estimated using a cross-validation model.

Results

DBS resulted in a mean CL and IL symptom reduction of $56.7 \pm 26.8 \%$ and $15.9 \pm 51.8 \%$, respectively. Successful interpolation was performed with good validation (R2>0.80). CL UE and LE symptom reduction was best associated with leads placed within the entire STN or the caudal ZI . However, IL UE symptom reduction was found in regions anterior and lateral to the STN. IL LE effect was limited to the regions within the central and posterior STN . Generally, IL UE reduction was found in more lateral regions, than LE effects, though significant overlap exists.

Conclusion

Based on these reconstructions, differing limb and laterality somatotopies within and around the STN. Further studies are required to determine if targeting for ipsilateral effect is a valid surgical planning strategy. A better spatial understanding of STN function may be critical in direct and customized targeting strategies. 
A Pilot Clinical Trial Evaluating Autologous Peripheral

Nerve Grafts Implanted into the Nucleus Basalis of

Meynert in Patients with Parkinson's Disease and Mild

Cognitive Impairment

Craig van Horne, Amelia Anderson, George Quintero, Julie Gurwell, Rich Lamb, Andrew Welleford, John Slevin, Greg A. Gerhardt

$\underline{\text { Introduction }}$

No treatment halts or modifies the course of clinical decline of motor and non-motor symptoms in Parkinson's disease (PD).

Cognitive impairment is one of the most troubling non-motor symptoms and is associated with a loss of cholinergic neurons in the nucleus baslalis of Meynert (NBM). Nerve growth factor (NGF) has been shown to support the cell maintenance and survival of cholinergic neurons in experimental models. We tested the safety and feasibility of transplanting autologous peripheral nerve grafts (APNGs), containing Schwann cells which have been shown to produce NGF, into the NBM in PD patients with mild cognitive impairment (NCT02369003). Grafts were placed at the time of bilateral deep brain stimulation (DBS).

Methods

Five subjects have participated currently. All had a diagnosis of idiopathic PD with MCI, demonstrated from a comprehensive pre-operative neurocognitive exam. Subjects were good candidates for bilateral DBS of the globus pallidus internus. APNGs were harvested from the sural nerve and deposited unilaterally into the NBM, targeted electrophysiologically, contralateral to the most symptomatic side. Subjects were followed clinically for adverse events. Post-op MRIs were obtained within 24 hours.

\section{$\underline{\text { Results }}$}

Subjects were successfully implanted with APNGs to the NBM. MRIs demonstrated safe graft implantations without hemorrhage or edema. There were no significant adverse effects related to graft implantation within the perioperative period. One subject has been evaluated postoperatively at 3 months and 8 months with follow-up neurocognitive evaluations showing stable diminished scores in verbal fluency tests (also observed in DBS patients) and substantial improvements in semantic fluency and psychomotor speed. Conclusion

Evidence is provided that our DBS Plus approach is safe and feasible. This is in agreement with our previous studies of APGNs implanted into the substantia nigra $(n=19)$. Results are preliminary. Full evaluations will be performed through 24 months.

\section{Dopamine Release in the Nonhuman Primate Caudate and Putamen Depends Upon Site of Stimulation in the Subthalamic Nucleus}

Erika K. Ross, Paul Hoon-Ki Min, Hang Joon Jo, Shinho Cho, Megan Settell, Ju Ho Jeong, Penny Duffy, Su-Youne Chang, Kevin Bennet, Charles Blaha, Kendall H. Lee

\section{Introduction}

Deep brain stimulation (DBS) of the subthalamic nucleus (STN) is an effective treatment for medically refractory Parkinson's disease. Although it has recognized clinical utility, its biologic mechanisms are not fully understood, and dopamine release as a potential factor in those mechanisms is in dispute. Here, we tested the hypothesis that STN DBSevoked dopamine release depends on the precise location of the stimulation site in the STN and site of recording in the caudate and putamen.

\section{Methods}

We conducted DBS with miniature, scaled-to-animal size, multi-contact electrodes and used fMRI to identify the optimal dopamine recording site in the brains of three non-human primates, which are highly representative of human brain anatomy and circuitry. Real-time stimulation-evoked dopamine release was monitored at carbon fiber microelectrodes using in vivo fast scan cyclic voltammetry. $\underline{\text { Results }}$ This study of STN DBS-evoked dopamine modulation in intact nonhuman primates used fMRI to identify the optimal recording site in the putamen and caudate and found a relationship between dopamine release in those structures and site of stimulation in the STN.

Conclusion

This study demonstrates that STN DBS-evoked dopamine release can be minimized or maximized through subtle changes in the STN stimulation site. 
Implantation of DBS Electrodes into GPi in Parkinson's Patients Under General Anesthesia: A Clinical Outcome Analysis

Nessa Susan Timoney, George Quintero, James Haywood Smith, Jr., Greg A. Gerhardt, Craig van Horne

$\underline{\text { Introduction }}$

Implantation of DBS electrodes is done while patients are awake or under general anesthesia (asleep), but asleep patient clinical outcomes are not fully documented. In addition, the procedures involved in DBS electrode placement under general anesthesia are not uniform. Our objective was to evaluate the effectiveness of "asleep" standardized GPi surgery with electrophysiological recording in PD patients, by assessing postoperative clinical outcomes and technical accuracy of lead placement through computer mapping. Methods

A retrospective review of 16 advanced PD patients who underwent bilateral GPi lead placement between 2013-2015 was performed. All patients underwent UPDRS assessment 618 months after DBS surgery. Preoperative functional measurements obtained as part of standard clinical care were compared to postoperative data. Post operative DBS system settings, clinical reports of efficacy and side effect profiles, and technical accuracy of lead placement, as measured by the mean radial error, were also analyzed.

$\underline{\text { Results }}$

Preliminary results include 16 participants (11 males, 5 females) with a mean age of 70.45 years at implantation. Mean time of postoperative testing was 11.26 months. UPDRS motor preoperative mean off-medication score was 53.26. UPDRS motor mean off-medication/on-stimulation score was 31.43. Mean UPDRS motor on-medication/on-stimulation score was 23.77. Preoperative mean on-medication UPDRS motor score was 28.7. The levodopa equivalent daily dose was reduced on average by $53.338 \%$. There were no adverse events attributable to electrode placement.

Conclusion

GPi leads placed while the patient is under general anesthesia using a standardized technique with electrophysiological recording resulted in improved clinical outcomes, consistent with the level of improvements reported previously for GPi electrode placements under awake conditions. As the study is currently ongoing, the preliminary results are promising and will greatly add to the sparse literature available in regards to DBS targeting GPi while under a general anesthesia.
An Unexpectedly High Rate of Revisions for Deep Brain Stimulation Surgery in North America

John David Rolston, Dario J. Englot, Philip A. Starr, Paul S. Larson

\section{Introduction}

While many studies describe outcomes and complications related to deep brain stimulation (DBS), the majority of these are from large academic centers, and results may differ from those in general neurosurgical practice.

Methods

Using data from the Centers for Medicare and Medicaid Services and the American College of Surgeons (ACS), we identified all DBS procedures related to primary placement, revision, or removal of intracranial electrodes.

$\underline{\text { Results }}$

Over 28,000 cases of DBS electrode placement, revision, and removal were identified during the years 2004-2013. Between $15.2 \%$ and $34.0 \%$ of these procedures were for electrode revision or removal. Significant predictors of revision and removal were decreased age (odds ratio (OR) of 0.96 ; $95 \%$ CI: $0.94,0.98)$ and higher ASA classification (OR 2.41; 95\% CI: $1.22,4.75)$. Up to $48.5 \%$ of revisions may have been due to improper targeting or lack of therapeutic effect.

Conclusion

Data from the multiple databases suggest that DBS has a higher rate of revision and removal than previously reported. There is a clear need to better track and understand the true prevalence and nature of such failures as they occur in the wider surgical community. 


\section{DIRECT DBS: A Prospective, Multi-Center Clinical Trial with Blinding for a Directional DBS Lead}

Jens Volkmann, Stephan Chabardes, Peter Schuurman, David Blum, G. Karl Steinke, Stephen Carcieri, Ljubomir Manola, Nic Van Dyck

\section{Introduction}

Historically, DBS systems have delivered stimulation using cylindrical electrodes, which stimulate neurons around the entire circumference of the lead. In this study, we will test a directional DBS lead, which includes radially segmented electrodes designed for selective stimulation in directions orthogonal to the lead trajectory, in addition to standard cylindrical electrodes. Bilateral directional DBS leads will be connected to the Boston Scientific Vercise PC pulse generator, which provides an independent current source for each of its 16 contacts. This system, therefore, is capable of current steering to shape stimulation in the plane orthogonal to the long axis of the lead ("directional stimulation"), as well as providing Ring Mode ("omnidirectional") stimulation equivalent to historical leads. We aim to characterize the effects of directional stimulation in subjects implanted with this system.

\section{Methods}

DIRECT DBS is a prospective, randomized, multi-center, double-blind study employing a crossover design. Subjects (10 to 12, adaptive) will be enrolled per center standard of care. Visits occur in 3 major periods - during implant, at 3 months, and at 1 year. Programming is restricted during the first 3 months post-implant to Ring Mode. At 3 months, multiple single-day programming visits will be undertaken to optimize directional programming. Patients are then randomized to one of two arms (4 weeks per arm) for a double-blind crossover comparison between Ring Mode and unrestricted (e.g. directional) programming. After the crossover phase, subjects enter an open-label phase of the study, with follow-up at 1 year.

\section{Results}

This exploratory study will have no prospective statistical hypothesis, but will collect data such as side effect threshold, therapeutic window, UPDRS scores, and quantitative accelerometer-based measures of bradykinesia and tremor. Conclusion

The DIRECT DBS trial will compare the effects of directional stimulation and omnidirectional stimulation over the first year post-implant. Results will inform future studies.
Correlation of the Tractography-based Target Motor Improvement in Deep Brain Stimulation for Patients with Parkinson's Disease

\section{Josue Moises Avecillas-Chasin, Juan A. Barcia}

\section{Introduction}

Localization of the dorsolateral zone (motor) of the subthalamic nucleus for deep brain stimulation (DBS) in patients with Parkinson's disease (PD) is possible with tractography through the connections from the M1/SMA. However, tractography has not been adequately validated for DBS targeting. In this work, we analyze the overlapping between the patient-specific volume of tissue activated (VTA) and the subthalamic target obtained by tractography correlated with the motor improvement in patients with PD.

Methods

We include 6 patients underwent bilateral STN-DBS (12 electrodes) with more than a year of followup. We obtained the motor zone of the STN using a method described by our group previously. Based in the work of Mc Intyre et al., we used the Optivise ${ }^{\circledR}$ software package to obtain the VTA of each clinically effective electrode's contact of every patient. We obtained the UPDRS III score of each side of the patients (12 scores). We computed the percentage of improvement based on the pre and postoperative scores. Finally, we obtained the percentage of overlapping between the VTA and tractographical target of the STN. We used the Spearman correlation to analyze the relationship between the VTA/motor STN and the percentage of motor improvement.

Results

The Wilcoxon test revealed a statistically significant improvement of all patients after STN-DBS ( $\mathrm{p}=0.0024)$. Correlation analysis showed a positive correlation between the $\mathrm{VTA} /$ motor STN and the percentage of motor improvement $(\mathrm{r}=0.58$; moderate correlation) with a statically significant result $(\mathrm{p}=0.048)$.

Conclusion

This study suggest that stimulation in the motor part of the STN obtained by tractography is associated with a better motor improvement than stimulation outside of the motor part of the STN. There is positive correlation between the electrical influence of this tractographical target with the degree of motor improvement. 


\section{Preliminary Experience with Chronic Directional DBS in the STN}

Claudio Pollo, Ines Debove, Julia Müllner, Lennart Lachenmayer, Markus Florian Oertel, Frédéric Rossi, Michael Schüpbach

Introduction

STN DBS has been shown to drastically improve motor symptoms of PD. However, the occurrence of disabling side effects may limit the benefit of the therapy. Computed models have suggested that directional stimulation could increase its efficacy. Intraoperative studies performed in human have shown that directional stimulation provides differents thresholds for clinical effects. In the present study, we investigate the effect of directional stimulation on beneficial and side effects, in chronically implanted patients compared to omnidirectional stimulation.

Methods

7 bilateral STN implanted PD patients have been prospectively included in this study. In the trajectory determined after microrecording and intraoperative clinical testing, the definitive directional lead (1-3-3-1 electrode configuration, Vercise, Boston Scientific) was implantated with one electrode oriented medially, one anterolaterally and the third posterolaterally, under intraoperative fluoroscopic control. Monopolar omnidirectional stimulation was initially performed. At least 1 month after surgery, directional stimulation was assessed. The current threshold for beneficial and side effects was assessed for each of the 3 directions and compared to omnidirectional stimulation.

$\underline{\text { Results }}$

So far 4 patients ( 8 sides) have been fully assessed. A best direction of stimulation was observed on all sides for positive effect on rigidity as well as thresholds of side effects. The current required to obtain a beneficial effect in the best direction varied from $50-100 \%$ of the one required in the omni-directional state The current required to achieve a sustained side effect in the worst direction was comparable to the in the omni-directional situation. The medially oriented directional electrode was found in 5/8 sides to have the highest threshold for side effects.

Conclusion

Our preliminary experience using Directional DBS in the STN performed postoperatively suggests the persistence of different thresholds for the appearance of clinical effects in directional stimulation conditions, compared to omnidirectional stimulation. Further data are needed to confirm these observations.
Prevalence of Twiddler's Syndrome May Be Higher in the Internal Pulse Generators Harboring One Anchoring Hole Than Two Anchoring Holes

Michael Roman Sobstyl, Miroslaw Zabek, Grazyna

Brzuszkiewicz-Kuzmicka, Tomasz Pasterski

Introduction

Twiddler syndrome (TS) is described as a spontaneous rotation or intentional external manipulation of implanted cardiac or occasionally deep brain stimulation (DBS) devices. The predisposing factors for development of TS include advanced age with more loose subcutaneous tissue, adipose patients with thick tissue layer precluding proper immobilization of internal pulse generator (IPG), creation of excessively large pocket, or obsessive-compulsive behavior. Additional factor related to the construction of the implanted hardware itself may be the number of anchoring holes in the IPG.

$\underline{\text { Methods }}$

A prospectively collected database of all hardware related complications for patients operated on at the Neurosurgical Department of Postgraduate Medical Center of Warsaw was performed. In a total number of 347 leads implanted in 211 patients since 1999 we have identified 3 patients diagnosed with TS. All 3 patients with TS in our series were implanted with the IPG harboring one anchoring hole. This complication did not occurred in patients with the IPG harboring two anchoring holes.

Results

All 3 patients underwent revision surgery. During reoperations all IPGs were replaced and sutured with one additional silk suture through the plastic housing of the IPG to immobilize it properly in subcutaneous pocket. There were no recurrences of TS in our patients. All patients gained the previously derived benefit from STN DBS.

Conclusion

Our case series suggests that a predisposing factor of TS may also be the construction of IPG itself (one anchoring hole intended for fixation) which naturally represents less fixation of the IPG to the fascia or muscle in the subcutaneous pocket. This preliminary report may favor the IPG with two anchoring holes which is less prone for development of TS. Placing additional silk suture that pass through a plastic housing of the IPG may help better immobilize the IPG and reduce the occurrence of TS. 
Spinal Cord Stimulation Improves Gait in Patients with Parkinson's Disease Previously Treated with Subthalamic Nucleus Deep Brain Stimulation

Carolina Pinto de Souza, Clement Hamani, Carolina De Oliveira Souza, William Lopez Contreras, Maria Gabriela Dos Santos Ghilardi, Rubens Gisbert Cury, Egberto Reis Barbosa, Manoel Jacobsen Teixeira, Erich Talamoni Fonoff

$\underline{\text { Introduction }}$

Dopaminergic medications and deep brain stimulation (DBS) are well-established treatments for controlling motor symptoms and improving quality of life in Parkinson's disease (PD).While these therapies ameliorate cardinal motor symptoms, their effects on postural instability and gait disturbance (PIGD) are not sustained at long-term. At present, the treatment of PD patients who continue to experience PIGD even after optimized medical therapy and DBS is considered quite challenging. This is of importance as falls associated with postural instability and gait disturbance are major sources of morbidity and mortality in advanced PD.

\section{Methods}

We conduct a phase 1 clinical trial in four PD patients who experienced significant PIGD after subthalamic DBS. Primary outcome were changes in gait 6 months after thoracic SCS at $300 \mathrm{~Hz}$ compared to baseline. These were measured with the Timed Up and Go test (TUG), TUG dual task (DT) and the 20-meter-walk test. Secondary outcome were changes in UPDRS III, freezing of gait (FOG-Q) and quality of life (PDQ-39). To confirm the effects of SCS, gait performance was measured in double-blinded experiments carried out at $300 \mathrm{~Hz}$ or $60 \mathrm{~Hz}$ (settings that generated equally perceived paresthesias). All evaluations were carried out while patients were receiving DBS.

\section{$\underline{\text { Results }}$}

Six months following SCS $300 \mathrm{~Hz}$, patients (OFF meds) had significant improvements in the TUG $(63.2 \%, \mathrm{p}=0.006)$ and the $20 \mathrm{~m}$-walking test $(58.0 \%$ decrease in time; $\mathrm{p}=0.05 ; 65.7 \%$ decrease in the number of steps, $\mathrm{p}<0.009$ ) as compared to preoperative baseline. In addition, patients experienced significant improvements in UPDRS III (38.3\% "OFF" meds $\mathrm{p}=0.034)$, FOG-Q (56.4\% "ON" meds, $\mathrm{p}<0.001)$ and PDQ-39 (44.7\% "ON" meds, $\mathrm{p}=0.002$; mobility). Blinded "OFF" medication "ON" DBS assessments confirmed primary outcome data, showing significant objective improvements in gait after $300 \mathrm{~Hz}$ SCS but not at $60 \mathrm{~Hz}$.

Conclusion

PD patients tolerated $300 \mathrm{~Hz}$ SCS very well, experiencing significant improvement in gait measures and quality of life.

\section{Rescue Lead Therapy in Patients with Essential Tremor Refractory to Single Array VIM DBS}

Vishad V. Sukul, David A. Isaacs, Srivatsan Pallavaram, William Rodriguez, Jonathan Butler, Hong Yu, Joseph Samir Neimat, Peter Konrad

Introduction

Nucleus ventralis intermedius (Vim) deep brain stimulation (DBS) is an effective therapy for the treatment of refractory essential tremor (ET). 9\% of patients may fail therapy after initial good results. Some suggest placement of an additional anterior adjacent lead can improve symptoms through electric field-steering. We describe here our cumulative surgical experience.

Methods

A total of 10 out of 297 Vim patients underwent revision, with 6 reviewed retrospectively for this study. These patients had a second DBS array placed after programming failed to recapture initial tremor control. Leads were implanted with the intent of steering the field anteriorly to recapture tremor control in the motor thalamus. Clinical, anatomic and intraoperative data were post-processed with respect to the probabilistic atlas.

$\underline{\text { Results }}$

A total of 9 leads were implanted in 6 patients ( 3 bilateral, 3 unilateral). The Vim cluster generated in the atlas shows expected overlap with the normalized efficacy map predicting good initial tremor capture relative to the cumulative ET population. Time to secondary implant was $2.68 \pm 1.38$ years with the exception of one outlier at 11 years. New leads were placed on average between $3-4 \mathrm{~mm}$ anterior and medial to the originals. Programming voltages were decreased from preop by $1.8 \pm 1.3 \mathrm{~V}$ in four hemispheres, increased by $0.9 \mathrm{~V} \pm 0.8 \mathrm{~V}$ in four, and unchanged in two. Rescue of tremor control was demonstrated in all patients based on clinical evaluation. Conclusion

Rescue lead therapy shows clinical benefit for tremor control in Vim DBS refractory ET patients. There is debate regarding why this occurs- electrical tolerance vs. physiologic 'escape' from capture by a single array lead. However, our results suggest that failed control with previously successful single array implants can be rescued by a second anterior array implant. Further investigation is ongoing and will allow us to further quantify this phenomena and therapeutic option. 


\section{DBS Stimulation Parameters for Dystonia - A Systematic Review}

Philippe Magown, Muhammad Sohail Noor, Zelma HT Kiss

$\underline{\text { Introduction }}$

Deep brain stimulation (DBS) of the globus pallidus internus (GPi) for dystonia was originally based on clinical improvement in case series in the face of a limited pathophysiological understanding of this movement disorder. Initially stimulation parameters were based on those used for Parkinson's disease (PD) and later higher parameters were recommended. Now after almost two decades of experience, various centres utilize higher and others, lower stimulation parameters. For example, if patients develop parkinsonism, some centres drop the frequencies applied. Because of these inconsistencies, we systematically reviewed the literature to identify the variability of stimulation parameters in dystonia. Methods

A comprehensive systematic literature search was performed on the following databases: Medline/PubMed, Embase and the Cochrane database of systematic reviews using the following MeSH terms or keywords: "deep brain stimulation" or "deep brain stimulation/methods", and "dystonia" or "dystonia/therapy". Publications were limited to human subjects and English language. Selected articles were classified based on details provided about outcomes and electrical parameters. From each selected publication, patient characteristics, stimulation parameters, follow-up period and clinical outcomes were extracted.

\section{Results}

Two hundred eighty nine publications were retrieved from Medline/PubMed. Twenty-six additional articles were identified through Embase. Publications were reviewed to extract the above criteria and demonstrate the variability of stimulation parameters used for DBS treatment of dystonia. Conclusion

DBS stimulus parameters were defined in the PD patient population and have been adapted for treatment of dystonia. Here we determine how DBS electrical parameters differ for the treatment of dystonia.

\section{Cost of Deep Brain Stimulation Explantation Secondary to Infection}

Tsinsue Chen, Margaret Lambert, Omar Gonzalez, Anna Moran, Andrew G. Shetter, Francisco A. Ponce

Introduction

Deep brain stimulation (DBS) hardware infection is a serious complication that may result in increased healthcare costs. Multiple hardware salvage attempts, numerous hospitalizations, and long term antibiotic therapies can result in a significant economic burden. We report the cost of DBS explantation secondary to infection at a single institution. Methods

362 patients underwent 540 electrode placements between January 1, 2010 and December 30, 2014. 15 patients (4.1\%) with complete explants due to infection were identified, and financial data was available for 13 patients. Demographic and financial data, including all itemized hospital costs and physician fees were analyzed.

$\underline{\text { Results }}$

The number of hardware salvage procedures ranged from 1-5 per patient (average $2.46 \pm 1.45$, median 2). $53.84 \%(n=7)$ of patients elected to undergo re-implantation after infection clearance. The average cost for a patient undergoing the median number of revisions (2), device explanation, and subsequent re-implantation after infection clearance was $\$ 75,504.17$. $53.1 \%$ of this cost was attributable to device reimplantation $(\$ 40,060 \pm 11,512.01)$. Operating room costs were the highest revenue category for hardware revisions and explants. There were no differences in cost categories between revisions and explants. Medical and surgical supplies incurred the highest cost for re-implantations. There were no recurrent infections or peri-operative deaths during the follow up period after re-implantation (25.25 months \pm 10.41$)$. Conclusion

DBS infection incurs significant healthcare costs associated with hardware salvage attempts, explants, and reimplantations. Operating room services and medical and surgical supplies were among the highest revenue categories for these procedures. 
Essential Tremor as a Deficit of Sensorimotor Integration

Jeewon Chon, Jean-Paul Noel, Michael Harvey, Joseph Samir Neimat

Introduction

As we navigate our world, our sensory receptors are activated in spatial and temporal patterns that are largely dependent on our own movements. Exploratory movements serve to localize, in a self-referential framework, biologically relevant features of the environment. In several movement disorders, e.g., essential tremor (ET), Parkinson's disease, dystonia, these intentional exploratory movement patterns are disrupted by unintentional movements that often have a stereotyped rhythmicity. These unintentional movements are not appropriately patterned in order to optimize the localization of a target, yet little is known of how this affects discriminative movement strategies, sensory discrimination, or its neurophysiological underpinnings. Here we test DBS implanted ET patients on a hand localization task while adjusting the frequency and amplitude of VIM stimulation.

\section{$\underline{\text { Methods }}$}

Implanted DBS ET patients, and age-matched controls, were recruited through patient databases. Participants were asked to guide a computer-mouse along a track and estimate the location of a target. They are unable to see their hand or cursor relative to the target. For DBS patients were tested both in the OFF-Stim condition and during epochs with stimulation variably set at 3 frequencies and 2 amplitudes.

\section{$\underline{\text { Results }}$}

While target detection accuracy was unaffected by stimulation, clear changes were observed in task kinematics in DBS both during different stimulation protocols and relative to control subjects.

Conclusion

Target detection accuracy was unaffected by VIM stimulation, with seemingly little to no effect of the frequency or amplitude of stimulation on task-performance. These findings call into question the role of motor thalamus in sensorimotor integration.

\section{Human Subthalamic Nucleus Activity Interacts with the Prefrontal Cortex to Gate Memory Encoding}

\author{
Baltazar Zavala, Anthony Jang, Kareem A. Zaghloul
}

Introduction

There is growing evidence that subthalamic nucleus (STN) theta $(4-8 \mathrm{~Hz})$ oscillations, together with those of the medial prefrontal cortex, play a role in preventing or delaying actions during high conflict decisions. The roles the STN may play in non-motor cognition, however, remain relatively unexplored. Here, we examined whether the coordinated activity between the prefrontal cortex and STN local field potential and spiking activity modulates the inhibition of memory.

Methods

We simultaneously captured STN single-unit spiking, STN local field potentials, and prefrontal cortex electrocorticography during DBS surgery as patients performed a novel working memory task. Subjects were sequentially shown eight numbers and asked to either encode or ignore each number based on a simultaneously presented shape.

$\underline{\text { Results }}$

During all correct trials, we observed a theta band specific increase in STN power, prefrontal power, and inter-site coherence between the STN and the prefrontal cortex. Crucially, all of these metrics showed a significantly higher increase during the ignore trials relative to the encode trials. Furthermore, the spiking of the STN neurons was also modulated during the task, with some neurons demonstrating an increase in firing and others a decrease. In the neurons that demonstrated a decrease, we found that this decrease was attenuated during the ignore trials.

Conclusion

In line with previously held notions that the STN acts as a gate that influences whether or not an action occurs, our results suggest that this gating mechanism may also extend to nonmotor actions such as memory encoding. Moreover, the gating mechanism involves the frequency specific interaction with higher-level cognitive areas such as the prefrontal cortex. These results provide insight into the cognitive deficits associated with STN pathology and deep brain stimulation. 


\section{Reward Expectation in the Subthalamic Nucleus}

Jonathan R. Flynn, Albert J. Fenoy, Harel Shouval

Introduction

The ability to make predictions about the environment is critical for an organism's survival, and the reward system in mammals is critical to this ability. Various reward centers have been shown to project to the subthalamic nucleus (STN), and recent evidence in animal models suggests that the region is involved with reward expectation. Our experiments extend these results and specifically test for reward expectation in the human STN.

\section{Methods}

Parkinson's Disease patients ( $n=9)$ were asked to participate in our study during microelectrode recording as part of the deep brain stimulation (DBS) procedure. Within confirmed ventral STN, patients were presented with a visual stimulus (a green dot), followed one second later by either a reward (several drops of sugar water/32 trials) or nothing ( 6 trials). The reward/non-reward conditions were analyzed and compared using spike rates, LFP data, and the RMS summary statistic using MATLAB.

\section{$\underline{\text { Results }}$}

LFP analysis has shown an unsurprising heterogeneous response. Multiple recordings demonstrate population activity correlated with the stimulus, the reward, or both in the Alpha and Beta bands. Other STN neurons appear to encode for prediction error in the same bands; i.e., the neurons respond when they receive an unexpected reward, or do not receive an expected reward.

\section{Conclusion}

The preliminary results are in line with previous non-human research - the STN appears to be related to reward expectation, with some sites showing activity when that expectation is violated. Further study is being done to link these results to the reward system as a whole, and the STN's place in it.

\section{Single Neuronal Correlates of Subjective Opinion in the Human Prefrontal Cortex}

\section{Ziev Moses, Mohsen Jamali, Ziv Williams}

Introduction

Subjective judgment allows humans to make rapid decisions under novel scenarios in the absence of explicit rules to guide their selection, and plays a critical role in many social, legal and political arenas. The single-neuronal basis by which subjective judgments are made in humans, however, is unknown.

Methods

Ten human subjects undergoing DBS surgery gave informed consent to participate in our study. Using microelectrodes, we isolated single units in the dorsolateral prefrontal cortex (DLPFC) along the trajectory of the clinical target. Patients then completed a subjective judgment task, where they are presented with visual stimuli of seven different real-world scenes with varying levels of danger and asked to judge whether the scene is safe or dangerous. The spiking activity of DLPFC neurons were collected and single units were then spike-sorted and analyzed offline.

$\underline{\text { Results }}$

We isolated 96 single units in ten patients. 44 units demonstrated significant task-modulation compared with baseline activity (before stimulus presentation). We find that while many neurons displayed biphasic responses that closely tracked the individual's opposing decisions, another prominent and largely distinct group of neurons closely tracked the equipoise or 'tipping-point' between the opposing decisions independently of their objective report. Taken together, these neurons provided a remarkably accurate representation of the individual's voting profile, while displaying little or no information about the actual motor response used to indicate their selections.

Conclusion

We identify a group of neurons in the DLPFC that encode subjective valuations in humans, and provide a novel understanding of how complex subjective judgments are computed at the single-neuronal and population-levels. Taken together, these findings may help elucidate how decision making is represented in the human brain and provide new insight into how to treat individuals with higher cognitive disorders. 


\section{Dynamics Between Alpha and High Gamma Cortical Activity Shape Decision-Making}

Sameer A. Sheth, Elliot Smith, Catherine Schevon, Guy M. McKhann, Saskia Haegens

Introduction

The alpha rhythm $(8-14 \mathrm{~Hz})$ is thought to reflect a mechanism of functional inhibition, regulating the engagement and disengagement of neuronal populations based on task demands. Broadband activity in the high gamma range (70$150 \mathrm{~Hz}$ ) is considered a correlate of multi-unit activity. We track activity within these two frequency bands across a range of cortical areas using electrocorticography recordings in human patients with intractable epilepsy performing a cognitive interference task that allows us to address various cognitive aspects including attention, perception, decisionmaking and the subsequent motor response.

\section{Methods}

Subjects performed the multi-source interference task (MSIT), which combines elements of the Stroop, Simon, and Eriksen flanker tasks. We recorded ECoG activity from 18 subjects with coverage over a broad range of frontal, temporal, and parietal regions.

\section{$\underline{\text { Results }}$}

We find a negative relationship between alpha power and broadband high gamma activity. Combined, these activation patterns reflect temporal tracking of task-engaged regions, with alpha decrease and broadband increase locked to specific task aspects. We report different categories of responses, distributed over cortex, including sites that only respond to the stimulus presentation, sites that only respond to the decision report and/or the feedback, and interestingly, sites that track the time on task. The latter allows prediction of behavioral performance in terms of reaction times. Furthermore, we find a small subset of sites that show modulation with task difficulty, predominantly in temporal areas, and modulation based on task history.

Conclusion

Our results support the notion that alpha reflects functional inhibition at the local neuronal population level, gating neuronal activity as reflected by broadband high gamma. Crucially, the combination of these signals correlates with various task aspects and allows us to predict behavioral performance. We provide evidence for the generality of this mechanism across cortical areas, including prefrontal cortex.
Trigeminal Nerve Stimulation for the Treatment of Mild Traumatic Brain Injury

Jean-Philippe Langevin, Esther Choi, Drorit Gaines, Scott Krahl, Daniel Silverman, Gholam Berenji

Introduction

Traumatic Brain Injury (TBI) patients show dysfunctional recruitment patterns of the frontal lobes when performing cognitive tasks. Regulation of frontal lobes activity through neuromodulation could improve cognitive performance and neuropsychiatric symptoms in the setting of chronic mild TBI (mTBI). Trigeminal nerve stimulation (TNS) is a non-invasive form of neuromodulation that has been shown to modulate several regions of the frontal and cingulate regions on PET scan. We studied the effects of TNS on the neurocognitive symptoms of mTBI in relation to resting PET scans. Methods

This early-phase clinical trial follows a prospective open label design. Patients suffering from chronic mTBI are enrolled and complete baseline assessments that include a resting-state FDG-PET scan along with neuropsychological testing and psychological scales. The patients are then instructed to apply a self-adhesive electrode above the eyebrows for eight hours per day for eight weeks. The electrode connects to a pulse generator that the subject adjusts to achieve a pleasant sensation over the forehead. The PET scan and the assessments are repeated at the conclusion of the trial. $\underline{\text { Results }}$

For the first two subjects who have completed the protocol, PET scans demonstrated significantly increased metabolism in Broca's area, dorsolateral prefrontal cortex, anterior cingulate cortex and medial frontal cortex. The mean changes above pre-treatment values in each of these regions ranged from $1.7 \%$ to $4.3 \%$ of whole-brain mean metabolism, and were associated with self-reported improvement in the language and memory domains as well as substantial reduction in posttraumatic headaches. Cerebral metabolism was reduced by approximately $2 \%$ of pre-treatment values in the bilateral amygdalae, with an associated improvement in anxiety levels as reported on the Beck Anxiety Inventory.

Conclusion

TNS may prove beneficial in mTBI by normalizing recruitment patterns of the frontal and cingulate cortices. These neuromodulatory changes may improve neuropsychiatric symptoms seen in patients suffering from chronic mTBI. 
Activity in Human Substantia Nigra During Sensorimotor Decision-making Task Reflects Motor Planning

John Thompson, Gidon Felsen, Aviva Abosch

$\underline{\text { Introduction }}$

Parkinson's disease (PD) is known to result in altered activity in basal ganglia motor regions, but such activity has not been studied under controlled conditions in which precise, goaldirected movements are cued by stimuli. The substantia nigra pars reticulata $(\mathrm{SNr})$ provides a convenient window into basal ganglia output, as it plays a similar role in orienting movements across mammalian species.

Methods

In this study, during the microelectrode recording portion of deep brain stimulation (DBS) surgery for PD, we recorded from single $\mathrm{SNr}$ neurons while subjects $(\mathrm{N}=3)$ engaged in a sensorimotor decision-making task (random motion dot task; RMDT).

\section{$\underline{\text { Results }}$}

We found that human $\mathrm{SNr}$ single unit activity reflects task variables associated with RMDT. In particular, $\mathrm{SNr}$ neurons show increased activity during motor planning as the subject prepares to execute an eye movement.

Conclusion

These data provide the first support for the role of $\mathrm{SNr}$, in humans, for initiating and controlling movements.

\section{Processing of Emotional vs Non-Emotional Cognitive Conflict}

Matthew Kamal Mian, Emad N. Eskandar

$\underline{\text { Introduction }}$

Conflict occurs when incompatible response tendencies are activated simultaneously. Evidence has linked the anterior cingulate cortex (ACC) to conflict detection and the lateral prefrontal cortex (PFC) to conflict resolution. Recently, functional imaging studies have suggested that a separate but overlapping network involving the amygdala may process conflict with emotional salience, but neurophysiologic data are lacking.

Methods

We recruited human subjects $(n=6)$ undergoing stereotactic depth electrode insertion for epilepsy to perform two similar, validated conflict tasks, one with emotional salience and one without. During these tasks, we recorded local field potentials (LFPs) in the ACC, PFC, and amygdala.

$\underline{\text { Results }}$

Subjects performed both tasks accurately ( $>98 \%$ correct) and showed expected conflict-induced response slowing $(\mathrm{p}<1 \mathrm{x}$ $10^{-18}$ for each). LFPs revealed robust task-related activity. In both tasks, LFPs at a majority of ACC and DLPFC sites were sensitive to conflict (ACC: $84 \%$ vs $57 \%$, DLPFC: $59 \%$ vs $74 \%$ ), and many discriminated the level of cognitive control (ACC: $42 \%$ vs $67 \%$, DLPFC: $62 \%$ vs $41 \%$ ), with the left hemisphere more active in conflict resolution than the right $(\mathrm{p}=0.04$ and $\mathrm{p}=0.05$ ). In the non-emotional task, the ACC preferentially performed a conflict monitoring vs resolving role ( $\mathrm{p}=0.001)$, and this effect was particularly evident on the left. In the emotional task, the amygdala was modestly sensitive to conflict (24\% of sites). In contrast to functional imaging studies, the amygdala discriminated emotional valence over conflict $(p=0.01)$, with a strong attendant effect of valence on reaction times $\left(\mathrm{p}=1 \times 10^{-9}\right)$.

Conclusion

Conflict monitoring and resolution are distributed over a similar frontal network in emotional and non-emotional tasks, with a bias towards left hemispheric dominance. While the amygdala indeed registers emotional conflict, responses are at least as selective for non-emotional conflict, and emotional content - rather than conflict - is preferentially represented. 


\section{Reward Phenomenology Elicited by Stimulation of the Nucleus Accumbens}

Victor Du, Erin Yeagle, Jose Herrero, Miklos Argyelan, Ashesh Mehta

$\underline{\text { Introduction }}$

The Nucleus Accumbens (NAc) is implicated in reward pathways regulating normal behavior, as well as in disease, such as addiction and OCD. Clinical experience suggests NAc stimulation is effective in treating disorders such as alcoholism and OCD. We describe our experience of stimulation in the vicinity of NAc in patients undergoing intracranial evaluation for epilepsy surgery and reward phenomena elicited.

Methods

Five patients with medically-refractory epilepsy were implanted with intracranial depth electrodes in the region of the NAc in order to localize ictal onset. Clinical functional mapping of brain regions was performed with currents from 1 $\mathrm{mA}$ to $4 \mathrm{~mA}$. Patients' behavior and subjective impressions during the stimulation sessions were videotaped. Connectivity of the NAc was quantified with DTI, resting-state fMRI, and cortico-cortical evoked potentials (CCEPs). We compared the anatomical locations of sites where NAc did not elicit reported sensations by determining each stimulus site's stereotactic coordinates.

\section{Results}

In one of four patients, stimulation of the right NAc area resulted in reward phenomenology. These were reported as a "happy feeling like winning a lottery ticket with a small prize," and other sensations. DTI, resting fMRI, and CCEPs demonstrated anatomical and functional connectivity between the stimulated site in the NAc area and distant cortical regions associated with complex pleasure sensations. Stimulated electrodes eliciting complex reward percepts were often within the white matter ventral to the NAc compared to sites where stimulation did not evoke percepts.

Conclusion

We report a novel instance in which stimulation of NAc as part of clinical evaluation for an epilepsy patient elicited complex sensations of reward. Our determination of the effective stimulus area's stereotactic coordinates, in addition to our maps of NAc connectivity in this patient grant us better understanding of NAc connectivity with related cortical regions that may serve as targets for noninvasive stimulation for therapeutic benefit.

\section{Recording and Stimulation of the Pathologic Brain Cavity Wall in a Rat Model for Thalamic Syndrome}

Philippe De Vloo, Janaki Raman Rangarajan, Els Crijns, Alexander Bertrand, Bart J. Nuttin

Introduction

The thalamic syndrome, first described by Dejerine and Roussy, is a central neuropathic pain syndrome occurring after thalamic stroke, often associated with a mild paresis. It is a form of central post-stroke pain. Treatment is challenging and often not satisfying.

Methods

30 rats were tested for thermal and mechanical pain and motor performance, and were then randomly allocated into a lesion group (L; electrolytic thalamic lesioning; $\mathrm{n}=22$ ) and a sham group ( $\mathrm{S}$; sham surgery; $\mathrm{n}=8$ ). Pain and motor tests were repeated weekly over the next 4 weeks. Next, after CT and MR imaging, 3 bipolar electrodes were implanted. $\mathrm{L}$ was randomly divided into a cavity wall electrode group (W; electrodes aiming for the ventral cavity wall; $\mathrm{n}=11$ ) and a random electrode group $(\mathrm{C}$; electrodes aiming for a random brain target not related to motor or pain behaviour; $n=11$ ). In $\mathrm{S}$, electrodes were implanted at the same coordinates as in $\mathrm{W}$. Motor tests were then repeated during deep brain stimulation (DBS; biphasic, $130 \mathrm{~Hz}, 200 \mu$ s at $0 \%-50 \%-75 \%-100 \%$ of the highest tolerated amplitude (HTA; amplitude above which side effects are observed)). Afterwards, local field potentials (LFPs) were recorded in resting state.

Results

After but not before lesioning, motor scores were significantly $(\mathrm{P}<.05)$ worse in L vs. S, while pain scores did not differ. In C, DBS at $50 \%, 75 \%$ or $100 \%$ HTA did not improve motor scores significantly as compared to $0 \%$ HTA in W or to DBS in C or S. LFPs obtained from identical anatomical locations in $\mathrm{C}$ and $\mathrm{S}$ rats differed significantly.

Conclusion

In a thalamic syndrome rat model with motor deficits but no mechanical or thermal hyperalgesia, the tested DBS parameters did not alleviate symptoms. 


\section{Long-Term Results of Motor Cortex Stimulation in} Chronic, Intractable Neuropathic Pain

\section{Byung-Chul Son}

\section{Introduction}

Although motor cortex stimulation (MCS) has been used for more than 20 years in the treatment of chronic neuropathic pain, there is still a debate on the efficacy of MCS.

\section{Methods}

To investigate the long-term results and the factors associated with long-term success in chronic MCS, 21 patients who underwent MCS trial were classified into central poststroke pain (CPSP), central pain of spinal cord injury (SCI pain), and peripheral neuropathic pain ( $\mathrm{PNeP}$ ), and we investigated the clinical factors associated with long-term success and degree of pain relief.

\section{Results}

Of the 21 patients, $16(76.2 \%)$ had a successful trial and underwent chronic MCS. In the long-term follow-up $(53 \pm 39$ months), only type of pain (CPSP and PNeP) was associated with long-term success defined as more than $30 \%$ pain relief compared with baseline ( $\mathrm{p}<0.05$, chi-square test). The difference in pain relief was not significant in SCI pain $(>0.05$, one-way ANOVA). The other variables did not show any significant influence in the long-term success and degree of pain relief ( $>0.05$, one-way ANOVA).

\section{Conclusion}

MCS was more effective in the treatment of chronic neuropathic pain of CPSP and PNeP than that of SCI pain in the long-term.

\section{Simultaneous Trial of Thalamic Deep Brain Stimulation and Motor Cortex Stimulation in Chronic, Intractable Neuropathic Pain}

\section{Byung-Chul Son}

\section{Introduction}

Both motor cortex stimulation (MCS) and deep brain stimulation (DBS) of the ventralis caudalis $(\mathrm{Vc})$ thalamus have been shown to be effective in chronic neuropathic pain and modulation of thalamic and thalamocortical activity is regarded as a possible mechanism. Although Vc DBS and MCS share a common analgesic mechanism, application of MCS and DBS is still considered empirical and there is no consensus on which one is better.

\section{$\underline{\text { Methods }}$}

We performed a simultaneous trial of thalamic Vc DBS and MCS in 9 patients with chronic neuropathic pain and investigated the results of the stimulation trial and long-term pain relief.

$\underline{\text { Results }}$

Of the 9 patients initially implanted with both DBS and MCS electrodes, $8(89 \%)$ had a successful trial. Six of these 8 patients $(75 \%)$ responded to MCS and two out of the 8 patients responded to Vc DBS. With long-term follow-up, the mean NRS decreased significantly $(\mathrm{p}<0.05)$. Percentage of pain relief in the chronic MCS group and chronic DBS group was $37.9 \pm 16.5 \%$ and $37.5 \%$, respectively, and there was no difference $(\mathrm{p}=0.157)$.

Conclusion

Considering the initial success rate and the less invasive nature of epidural MCS than DBS, we think MCS would be more reasonable initial means of trial in chronic intractable neuropathic pain. 
Comparison of Clinical Efficacy and Computed

Tomography Analysis of Lead Position Between Three-

Column and Five-Column Paddle Leads Spinal Cord

Stimulation for Failed Back Surgery Syndrome

\section{Sangwoo Ha, Byung-Chul Son}

\section{Introduction}

The authors investigated the difference in clinical outcome and the position of paddle lead spinal cord stimulation (SCS) between three-column and five-column paddle lead SCS in patients with FBSS.

\section{Methods}

In 21 patients who underwent paddle lead SCS at T9 (threecolumn [ $n=12]$ and five-column [ $n=9]$ ) for FBSS, a 12-month follow-up numerical rating scale, percent pain relief, and CT assessment of contact angle and percent reduction of $\mathrm{T} 9$ canal area were investigated.

\section{Results}

There was no difference in paresthesia coverage of the painful area, trial success rate, clinical outcomes, and percent pain relief between the two groups ( $p>0.05$ ). Although there was no statistical difference in the contact angles, the contact angle in the five-column group was generally greater than that of the three-column group ( $\mathrm{p}=0.067)$. Overall reduction of $35.51 \pm$ $4.76 \%$ in the T9 canal was observed and there was no difference between two groups ( $p>0.05)$ and no correlation between the contact angle and percent $\mathrm{T} 9$ spinal canal reduction $(r=-0.247, \mathrm{p}>0.05)$.

Conclusion

There was no difference in clinical efficacy of SCS using three and five-column paddle lead. Significant inclination of paddle lead in posterior epidural space and significant reduction in T9 canal area were observed.

\section{Multimodal, Intraoperative Monitoring During Paddle Lead Placement for Cervicothoracic Spinal Cord Stimulation}

Jin-gyu Choi, Byung-Chul Son

\section{Introduction}

We investigated the efficacy of combined somatosensory evoked potentials (SSEP) and electromyography monitoring during paddle lead placement through cervicothoracic laminectomy under general anesthesia in a retrospective review of data from 25 patients.

$\underline{\text { Methods }}$

Muscle motor evoked potentials (MEP) recordings and SSEP monitoring were used for surveillance of the spinal cord. Collision testing of SSEP and threshold amplitudes of compound muscle action potentials (CMAP) in the bilateral upper and lower extremities evoked by electrode contacts of the paddle lead were checked to determine the laterality of the lead in the mediolateral direction.

$\underline{\text { Results }}$

A significant decrease in amplitudes of muscle MEP in spite of stable SSEP occurred in 2 patients: 1 patient with a retrograde $\mathrm{C} 1-\mathrm{C} 2$ insertion and another patient with an anterograde $\mathrm{C} 4 / \mathrm{C} 5$ insertion. Repositioning of leads based on significantly asymmetrical collision testing of SSEP and thresholds of CMAP in bilateral extremities was needed in 6 and 8 patients, respectively. In 22 patients, paresthesia coverage of the painful area was consistently located in the painful side, either unilaterally or bilaterally. There was no episode of revision for suboptimal lead placement.

\section{Conclusion}

Intraoperative neurophysiological guidance using SSEP and muscle MEP was useful for the safe and accurate placement of paddle leads for cervicothoracic SCS. 


\section{Pain Relief in CRPS-II after Spinal Cord and Motor Cortex Simultaneous Dual Stimulation}

Angelo R. C. Azevedo, William Omar Contreras Lopez, Manoel Jacobsen Teixeira, Erich Talamoni Fonoff

Introduction

We describe a case of a 30-year-old woman who suffered a traumatic injury of the right brachial plexus developing severe complex regional pain syndrome type II (CRPS-II). After clinical treatment failure, spinal cord stimulation (SCS) was used with initial positive pain control. After 2 years, pain returned progressively to almost baseline intensity before SCS. Additional motor cortex electrode implant was then proposed as a rescue therapy and connected to the same pulse generator.

Methods

This method allowed simultaneous stimulation of the motor cortex and SCS in cycling mode with independent stimulation parameters in each site. A blinded trial confirmed MCS effect on pain control programming the MCS to the minimum possible values until it was turned off for 1 week, leaving the SCS turned on.

Results

At 2 years follow-up, the patient reported an improvement of pain from $8 / 10$ to $3 / 10(60 \%)$ in the VAS with double stimulation (SCS plus MCS) and improved quality of life, SF36 score of 121 points (42\%).

Conclusion

The encouraging results in this case suggests that this can be an option as add-on therapy over spinal cord stimulation as a possible rescue therapy in the management of CRPS-II. How ever comparative studies must be performed in order to determine the effectiveness of this therapy.

\section{Low Back Pain Relief with a New 32-Contact Surgical Lead and Neural Targeting Algorithm}

Julie G. Pilitsis, Giancarlo Barolat, Joshua M. Rosenow, James J. Brennan, Alexander S. Bailey, Jeffrey M. Epstein, Blake Hammond, Clark Metzger, Dat Huynh, Kristen Lechleiter, Nitzan Mekel-Bobrov

Introduction

Treatment of low-back pain using Spinal Cord Stimulation (SCS) has been historically challenging. Advances in surgical leads and programming capabilities have been thought to potentially enable improved low-back pain relief using SCS. A recently introduced 32-contact surgical lead, which couples multiple independent current control (MICC) and anatomically-based neural targeting stimulation algorithms, allows for patient-specific programming optimization. We present here a real world, observational study of this 32contact surgical lead.

Methods

A multi-center, consecutive, observational study of a new 32contact surgical lead was carried out, using the Precision Spectra SCS System (Boston Scientific) in 100 subjects out to 12 months post-implant. We examined medical history, procedural information, programming parameters, and clinical outcomes including pain reduction (NRS), activities of daily living, and change in pain medications.

$\underline{\text { Results }}$

Surgical lead placement distribution was between T7 and L2, with most at the top of T9 (26\%). A mean reduction of 5.1 points (SD 2.15, p <0.001) from 7.8 (baseline) to 2.6 in overall pain was observed. A subset of subjects reporting low-back pain only exhibited a mean decrease of 6.0 points (SD 2.12, $\mathrm{p}<0.001$ ) from 8.3 (baseline) to 2.2. Of these, $83.1 \%$ showed $=50 \%$ back pain reduction. Increases in activities of daily living and reduction in pain medication usage were also observed in a majority of subjects.

Conclusion

Subjects implanted with a 32-contact surgical lead using a neural targeting algorithm demonstrated significant low-back pain reduction. These results support the postulate that advanced surgical leads and programming capabilities can foster improved low-back pain relief in subjects treated using SCS. 


\section{Outcome Prediction in Patients with Trigeminal Neuralgia Treated with Novalis Radiosurgery: An Image Analysis Study}

\section{Wei-Lun Lo, Nader Pouratian}

Introduction

Novalis ${ }^{\circledR}$ radiosurgery (NRS) is a stereotactic radiosurgery (SRS) modality used to manage trigeminal neuralgia (TN). Neurovascular conflict (NVC) is an important etiological cause of TN. The aim of this study is to determine the relationship between anatomical characteristics and treatment response to NRS for patients with TN.

Methods

This is a retrospective review of patients with TN treated by NRS at the University of California, Los Angeles Health System from 1996 to 2012. Two hundred and eighteen patients had high resolution T2 magnetic resonance imaging (HR-MRI) from which serial anatomical characteristics such as presence of $\mathrm{NVC}$, location of NVC, presence of vertebrobasilar dolichoectasia (VBD), distance between root entry zone (REZ) of trigeminal nerves and Meckel's cave, and diameter of vessels over NVC were derived. Patient charts were reviewed to obtain clinical outcomes and stratified by Barrow Neurological Institute (BNI) scores. Anatomical differences were evaluated for the trigeminal nerves on both the affected and non-affected sides.

Results

After NRS, treatment response decreased rapidly within six months and stabilized thereafter. The incidences of NVC and VBD on the affected side were significantly higher than the non-affected side (NVC: $p<0.001$; VBE: $p<0.001$ ). In terms of trigeminal nerve length within the prepontine cistern, the length of the affected side was shorter than non-affected side $(\mathrm{p}<0.01)$. Univariate analysis showed that the patients without NVC had more unfavorable short-term outcome than that of patients with NVC ( $\mathrm{p}=0.001)$.

Conclusion

NRS is a favorable treatment option for trigeminal neuralgia. When compared to patients with NVC, patients without visible NVC on preoperative MRI have poorer short-term outcome. However, the selection of SRS target might be important for treatment response, for which further investigation may be needed.

\section{Comparison of Percutaneous Retrograssean Balloon Compression and Gamma Knife Radiosurgery for the Treatment of Trigeminal Neuralgia in Multiple Sclerosis}

Aizik L. Wolf, Andres M. Alvarez-Pinzon, Sammie Coy, Jose E. Valerio

Introduction

Trigeminal neuralgia (TN) is characterized by brief paroxysmal unilateral facial pain that is severe.In patients with multiple sclerosis (MS), TN is attributed to demyelinating lesions of the central trigeminal pathways or the trigeminal root entry zone (REZ). The management for TN in MS must be adapted to each individual patient. The aim of this clinical research study was to evaluate and compare percutaneous retrogasserian balloon compression (PBC) and Gamma Knife radiosurgery (GKRS) in terms of effectiveness, complications and technical aspects in MS population.

Methods

This is a single center, retrospective, comparative study. Minimum follow up duration is 24 months. Patients with history of microvascular decompression or other previous intervention for the management of TN were excluded. Seventy eight PBC procedures and one hundred twenty four first dosage GKRS were performed by one experienced neurosurgeon in a total of 202 patients between February 2009 and December 2013. The PBC procedures were completed in all cases. The 2 groups compared initial effect, duration of effect, and rates of complications as well as severity and type of complications.

Results

The rates for immediate pain relief were $72 \%$ for patients treated with PBC and 35\% for patients treated with GKRS. The Kaplan-Meier plots for the 2 treatment modalities were similar. The 50\% recurrence time was 12 months for the PBC and 18 months for the GKRS. The rates of complications (excluding numbness) were 3\% for GKRS and $21 \%$ for PBC, and this difference was statistically significant (chi-square test, $\mathrm{p}=0.03)$.

Conclusion

Both PBC and GKRS are effective techniques for the treatment of trigeminal neuralgia in MS. GKRS presents advantages in terms of complications, long term relief and allowing treatment without general anesthesia. Authors consider GKRS first option for the treatment of TN in MS patients. PBC is reserved for patients in whom the effect of GKRS has proven to be short or due to acute intractable pain. 
Clinical Efficacy of Pulsed Radiofrequency Neuromodulation for Intractable Meralgia Paresthetica

Hyuj Jai Choi

$\underline{\text { Introduction }}$

Meralgia paresthetica (MP) is a neurologic disorder of the lateral femoral cutaneous nerve (LFCN), which is characterized by a localized area of paresthesia and numbness on the anterolateral aspect of the thigh. In most patients with MP, symptoms can be successfully managed with conservative treatment. However, in a small group of MP patients who are refractory to medical treatment, more aggressive low-risk treatment should be considered. The objective of this study was to evaluate clinical outcomes of pulsed radiofrequency (PRF) neuromodulation of the LFCN in MP patients refractory to conservative treatment.

Methods

We retrospectively reviewed the clinical data of 11 patients with medically intractable MP who underwent PRF neuromodulation of the LFCN. These patients with MP underwent a diagnostic LFCN block using 2.0\% lidocaine. Temporary pain relief $>50 \%$ was considered to be a positive response to the diagnostic nerve block. Following a positive response to the diagnostic nerve block, patients underwent PRF neuromodulation at $42^{\circ} \mathrm{C}$ for 2 min. Patients' pain was evaluated using a $10-\mathrm{cm}$ visual analog scale (VAS). In MP patients who received PRF, we statistically evaluated VAS scores and the presence of any complications for 6 or more months after the procedure.

\section{Results}

The mean initial patient VAS score was $6.4 \pm 0.97 \mathrm{~cm}$. This score was decreased to $0.91 \pm 0.70 \mathrm{~cm}, 0.82 \pm 0.75 \mathrm{~cm}$, and $0.63 \pm 0.90 \mathrm{~cm}$ at the 1-, 3-, and 6- month follow-ups, respectively $(\mathrm{P}<0.001)$. Sixty-four percent of patients achieved complete pain relief (pain- free) in the last follow-up, whereas $27 \%$ of patients achieved successful pain relief $(=50 \%$ reduction in pain as determined by the VAS score). Furthermore, we did not observe any complications after the procedure.

Conclusion

PRF neuromodulation of the LFCN provides immediate and long-lasting pain relief without complications. Therefore, PRF of the LCFN can be used as an alternative treatment in patients with MP who are refractory to conservative medical treatment.

\section{Restoration of Efficacy of Spinal Cord timulation after Conversion from Percutaneous to Paddle Leads}

\author{
Chen Xu, Raj Kiran Nangunoori, Nestor Denys Tomycz
}

Introduction

Spinal cord stimulation (SCS) is widely used for conditions such as failed back surgery syndrome (FBSS) and complex regional pain syndrome (CRPS I and II). While percutaneous or paddle lead systems are both viable options, percutaneous lead systems have a tendency to lose efficacy due to a higher migration rate, resulting in loss of clinical efficacy.

Methods

A retrospective chart review was conducted to identify patients who had lost efficacy from their percutaneous SCS systems and were converted to a paddle lead system, regardless of etiology.

Results

From 2013 to 2016, 7 patients were identified with a mean age of 54.8 years (range: $32-80$ ) who had received benefit from percutaneous lead systems and presented for evaluation with loss of efficacy. A total of 5 male and 2 female patients underwent conversion from percutaneous to paddle lead systems with a restoration and/or improvement in their symptom coverage. Diagnoses included FBSS $(71.43 \%)$ and CRPS (28.57\%), with a mean follow-up duration of 21 months (range: 2-36 months).

Conclusion

Failure of percutaneous SCS stimulation should not deter using SCS as a treatment modality for patients with a recurrence of their symptoms. While patients should be evaluated on a case-by-case basis, conversion to a paddle-lead system may allow for recapture of their clinical benefit and improve quality of life. 


\section{Occipital Nerve Stimulation for Non-Migrainous Chronic Headaches: A Systematic Review and Meta-Analysis Protocol}

Sylvine Carrondo Cottin, Léo Cantin, Michel Prudhomme

Introduction

Defined as a headache lasting at least 15 days per month, chronic headache is reported by $3 \%$ of the general population, and a substantial proportion of them are refractory to current therapies. Occipital nerve stimulation (ONS) is a treatment option, but is still considered as a last resort treatment mainly because of its invasive nature and the associated high cost. The aim of this review is to assess the efficacy and safety of ONS in regards to non-migrainous chronic headache.

Methods

We have conducted a systematic review with meta-analysis of patients with chronic headache excluding migraine treated by ONS. MEDLINE, Embase, and the Cochrane Library databases were searched for eligible studies. ONS was compared to sham stimulation or the best available treatment including injections, ablative techniques, and pharmacological or psychological interventions. Two independent reviewers processed to the screening of studies according to titles, abstracts, and then full texts. Using a standardized form, reviewers extracted the data from selected studies.

Results

The primary outcome is overall reduction of head pain severity. The secondary outcomes includes rates of reduction in severity of head pain, headache frequency, and duration, use of medication, quality of life, and adverse events. The risk of bias and the heterogeneity of the studies were also independently assessed based on the Cochrane Collaboration tool.

Conclusion

This systematic review will allow us to better assess the potential role of ONS for the treatment of patients with chronic headache excluding migraines that are refractory to less invasive therapies.
Thoracic Spinal Cord Stimulation in Patients with Morbid Obesity: A Case Series and Technical Considerations

Chen Xu, Raj Kiran Nangunoori, Nestor Denys Tomycz

Introduction

There is increasing evidence that patients with obesity, particularly morbid obesity (body mass index > 40), are at greater risk of complications with spinal decompression and fusion surgery. There is a dearth of literature analyzing the risks of complications in morbidly obese patients undergoing neuromodulation surgery such as spinal cord stimulation (SCS).

Methods

A retrospective chart review was conducted to identify patients with morbid obesity who had undergone thoracic SCS by a single-surgeon.

Results

From 2013 to 2016, 7 patients with morbid obesity were identified with a mean age of 51.71 years (range: $41-65$ ) and mean BMI of 43.24 (range: 39.1-51.5) who had undergone thoracic paddle lead spinal cord stimulation implant with flank pulse generator placement. All patients underwent general anesthesia with SSEP monitoring for thoracic SCS placement. Two patients underwent implant of Boston Scientific SCS, 4 patients underwent implant of St. Jude SCS, and 1 patient received a Medtronic SCS. Diagnoses included failed back surgery syndrome, CRPS, and occipital neuralgia. No patient developed infection or neurologic deficit but one patient had a culture-negative pulse generator wound dehiscence which required pulse generator explant. Surgery required larger instruments and retractors as well as longer operative times. Conclusion

Patients with morbid obesity can safely undergo placement of various thoracic SCS paddle lead systems. Based on this single-center, single-surgeon experience, patients with morbid obesity undergoing thoracic SCS are likely at higher risk for wound complications but can achieve similar efficacy outcomes as normal weight patients. Operative technical and anesthetic considerations of thoracic SCS surgery in morbidly obese patients will be presented. 


\section{AUTHOR INDEX}

Abosch, A.

Abulseoud, O.

Acharya, V.

Achey, R.L.

Ackerman, M.

Acosta, F.

Agrawal, P.

Ahn, M.

Air, E.L.

AlBakry, A.

Alesch, F.

Alhussein, A.

Alvarez-Pinzon, A.M

Anderson, A.

Anderson, D.

Angle, C.

Annetta, N.

Argyelan, $\mathrm{M}$.

Arza, R.

Asaad, W.

Auada, A.

Avecillas-Chasin, J.M.

Azevedo, A.R.C.

Bagher-Ebadian, H.

Bailey, A.S.

Barba, A.

Barbe, M.T.

Barcia, J.A.

Barolat, G.

Barros, G.

Basu, S.

Batista, L.

Batton, A.D.

Belasen, A.

Bennet, $\mathrm{K}$.

Bentley, J.N.

Berenji, G.

Bergman, $\mathrm{H}$.

Beristain, $\mathrm{X}$.

Bertrand, A.

Bijanki, K.R.

Black, K.

Blaha, C.

Blum, D.

Bockbrader, M.

$25,31,43,64$
24
18
39
16
51
10
54
6
40
35
54
69
55
33
45
19
23,65
5

53,54

9

5, 16, 57

34, 68

6

68

36

35

5, 16, 57

68

49

38

34

20

10

20, 55

36,48

63

25

33

65

32

17

55

57

19
Bond, A.E. $\quad 34$

Boulay, C. 19

Bouton, C. 19

Bowyer S 6

Brandmeir, N.J. $\quad 18,47$

Brennan, J.J. 68

Brucke, T. 35

Brzuszkiewicz-Kuzmicka, G. $\quad 41,45,58$

Bullis, C. $\quad 42$

Burchiel, K.J. $\quad 38,40,42$

Butler, J. $\quad 59$

Cabral, J. 27, 29

Cacciotti, G. $\quad 43$

Cantin, L. $\quad 71$

Caputi, F. 43

Carcieri, S. $\quad 57$

Chabardes, S. $\quad 57$

Chan, A. $\quad 17,26$

Chang, E.F. 21

Chang, S.-Y. 55

Chapple, K. 12, 52

Chaturvedi, A. 2

Chen, G. 8

Chen, J. 5

Chen, K.S. $\quad 48$

Chen, L. 35

Chen, T. $12,52,60$

Chenji, G. $\quad 51$

Chestek, C.A. $\quad 48$

Chivukula, S. $\quad 41$

Cho, C. $\quad 14$

Cho, S. 24, 55

Chockalingam, A. 36

Choi, E. 63

Choi, H.J. $\quad 70$

Choi, J.-G. 67

Chon, J. 61

Chou, K. 2, 2, 36, 51

Collison, C. 36

Colpan, E.M. $\quad 44$

Conrad, E. 2

Contreras Lopez, W.O. 34, 68

Cottin, S.C. 71

Coy, S. 69

Crijns, E. 65 
Crowell, A.L.

Cummings, $\mathrm{K}$.

Cury, R.G.

Danish, S.F.

Davidson, B.

$15,18,46$

52

Davis, $\mathrm{T}$.

Davoodi-Bojd, E.

Dawe, R.J.

de Andrade, E.M.

11

9

de Hemptinne, $\mathrm{C}$.

De Oliveira Souza, C.

1

De Vloo, P.

59

Debove, I.

Deeb, W.

Deogaonkar, M.

50

19

Dhall, R.

Di Scipio, E.

12

43

Ding, K.

28

Donahue, D.J.

30

Dos Santos Ghilardi, M.G. $\quad 59$

Dougherty, D.D.

4

Dowd, R.

50

Doyle, W.

Drees, $C$.

$\mathrm{Du}, \mathrm{V}$.

Duchin, Y.

Duenas, S.M.

Duffy, $P$.

Dugan, $\mathrm{P}$.

Durphy, J.

Dyster, T.G.

Eisenberg, H.M.

Eisenberg, M.B.

Elias, W.J.

Elisevich, K.

Englot, D.J.

Epstein, J.M.

Eskandar, E.N.

Farris, $\mathrm{S}$.

Felmlee, J.

Felsen, G.

Fenoy, A.J.

Flynn, J.R.

Fonoff, E.T.

Fountas, K.N.

Franklin, R.
Friedenberg, D. 19

Gaillard, W.

Gaines, D.

Gallay, M.N.

3

Garcia-Albea, J. 5

Garg, R.

Gee, L.

10,36

Georgiadis, I.

8

Gerhardt, G.A.

55,56

Gerrard, J.L.

49

Ghaly, M.

17

Ghanouni, P.

Giacobbe, P.

Giannis, T.

Gibson, W.

Gill, S.

Gilmour, A.

Giorgione, V.

Giroux, M.

Glenn, B.

Goble, T.J.

Gong, F.L.

Gonzalez, O.

Gooderham, P.A.

Gorny, K.R.

Grahnke, K.

Grant, J.

Greenlee, J.D.W.

Grobelny, B.T.

Gross, R.E.

Guan, Z.

Guerin, J.

Gunalan, K.

Gupta, K.

Gurwell, J.

Guthrie, B.L.

Gwinn, R.

$\mathrm{Ha}, \mathrm{S}$.

Haegelen, C.

Haegens, S.

Hamani, C.

Hammond, B.

Hanrahan, S.J.

Hanspal, E.

Harel, N.

Hargreaves, E.L.
3

4

8

24

35

4

4

52

19

49

9

60

47

24

33

42

32

6

$28,28,32,33$

36

54

11

31

55

54

3,7

67

35

63

59

68

52

36,40

11

15 
Harvey, M. 61

Haug, A. $\quad 52$

Haukas, A. 48

Hebb, A.O.

Hernandez, A.

Hernandez, R.N.

Herrero, J.

Hill, J.P.

Hill, T.

Ho, C.-Y.

Hobson, E.

Holland, M.

Holloway, K.L.

Holste, K.

Honey, C.

Honeycutt, J.H.

House, P.A.

Houshmand, L.

Howell, B.

Huang, J.

Huynh, D.

Hwang, R.S.

Ibrahim, A.

Inman, C.S.

Isaacs, D.A.

Ivan, M.E.

Ivanishvili, Z

Jacobs, C.

Jagid, J.R.

Jain, R.

Jamali, M.

Jang, A.

Jang, D.P.

Jeanmonod, D.

Jeong, J.H.

Jermakowicz, W.J.

Jo, H.J.

Jones, S.R.

Kaderi, T.

Kanner, A.

Kaprealian, T.B.

Kapsalaki, E.Z.

Kapur, R.

Keating, R.F.

Keator, C.

Keebaugh, A.
25,52

30

18

61

48

48

(

6

22

40

32

44

42

47

51

29

2

11,15

40

68

40

38

32

59

30

47

48

30

35

62

61

20

3

55

30

55

53

40

30

41

8

7

22

30

33
Keen, M. 33

Khalsa, S.S.S. $\quad 48$

Khan, A.R. $\quad 14$

Kimble, C.J. 20

Kiss, Z.H.T. 60

Klassen, B.T. 24

Knisely, J.P.S. $\quad 17$

Knopp, M.V. 10

Knowlton, R.C. 21

Ko, A.L. $\quad 38$

Kobylarek, M. 48

Kocak, M. 11

Kochanski, R.B. 39

Koduri, S. $\quad 45$

Koek, R. 5

Kohn, N. 1

Konrad, P. 12, 19

Kopell, B.H. 12, 14

Kovach, C.K. 32

Krahl, S. $\quad 5,63$

Kulick, A. 5

Kumar, R. $\quad 52$

Kurpad, S.N. $\quad 8$

Lachenmayer, L. $\quad 58$

Lamb, R. $\quad 55$

Lambert, M. $\quad 12,52,60$

Lamm, J.R. $\quad 37$

Lang, M.J. 21, 23, 49

Langevin, J.-P. $\quad 5,63$

Larson, P.S. $\quad 56$

Lau, J.C. 14

Lebrun, I. 9

Lechleiter, K. $\quad 68$

Lee, B.S. $\quad 39$

Lee, K.H. 20, 24, 55

Lee, $\mathrm{S}$. $\quad 53,54$

Lempka, S.F. 20

Li, S.-J. 8

Lin, D. $\quad 1$

Lipsman, N. 3, 4

Lo, W.-L. $\quad 41,69$

Lobel, D.A. $\quad 39$

London, D. $\quad 6,13$

Lopes, P. 9

Lopez Contreras, W. 59

Lozano, A.M. 4 
Lujan, L.J. 20

Maarouf, M. $\quad 35$

MacDougall, K. $\quad 14$

Machado, A. 39

Magown, P. 60

Mahmoudi, F. 6

Mahoor, M. 52

Malik, S. 30

Maling, N. 20

Mandelkern, M. 5

Manola, L. $\quad 57$

Markowitz, D.J. 1

Marks, W. 51

Marola, O. 46

Martinez, R. 9

Masnyk, T. 37

Mastronardi, L. $\quad 43$

Mayberg, H.S. 32

McCormick, P. Jr. 4

McInerney, J. $\quad 47$

McIntyre, C.

McKhann, G.M. 63

Megevand, P. 23

Mehta, A.

$7,23,65$

Mekel-Bobrov, N. $\quad 68$

Mercury, M. $\quad 37$

Metzger, C. 68

Mian, M.K. 64

Miller, A.M. 13

Min, P.H.-K.

Miocinovic, $\mathrm{S}$.

Mirzadeh, Z.

Mogilner, A.Y.

Molho, E.

Monette, A.

Moran, A.

Moran, J.E.

Morgan, A.

Morrell, M.J.

Morrison, M.

Moses, Z.

Müllner, J.

Murnick, J.

Myseros, J.S.

Mysiw, J.

Nagel, S.J.
Nangunoori, R.K.

Nazem-Zadeh, M.-R.

$45,70,71$

Nedrud, J.

Nehrbass, E.

Neimat, J.S.

Nencka, A.

Nielson, D.

Noel, J.-P.

Nolt, M.J.

Noor, M.S.

North, E.

Nuttin, B.J.

Oertel, M.F.

Ojemann, $\mathrm{S}$.

Okun, M.S.

Oluigbo, C.

Ostrem, J.L.

Oukal, S.

Owusu, S.A.

Ozawa, B.

Ozturk, G.

Paek, S.

Pagano, R.

Pahapill, P.A.

Pallavaram, S.

Palys, V.

Pantoli, D.

Parisi, A.

Parrent, A.G.

Pascual-Leone, A.

Pasterski, T.

Patel, N.V.

Patel, P.D.

Pathak, Y.

Patil, P.G.

Patriat, R.

Paulo, D.L.

Pereira, M.

Perry, M.S.

Peters, T.M.

Pilitsis, J.G.

Pineda-Pardo, J.A.

Pinto de Souza, C.

Polasani, R.

Pollo, C.

Ponce, F.A.
59,61

8

10

61

37,48

60

6

65

58

$25,31,43$

50

22

1

25

46

26

40

20

9

8

59

44

43

15

14

16

$41,45,58$

15

18,46

25

$2,2,36,48,51$

11

4

34

30

14

$10,36,40,46,68$

5

59

37

58

$12,52,60$ 
Porta, M.

Pouratian, N.

Pourfar, M.

Prudhomme, M.

Prusik, J.

Pun, J.

Quintero, G.

Racareanu, R.

Radwan, H.

Ralston, A.K.

Ramdhani, R.

Ramirez-Zamora, A.

Rangarajan, J.R.

Rao, A.J.

Raslan, A.M.

Rasouli, J.J.

Reddy, C.G.

Reis Barbosa, E.

Reitano, C.

Remer, D.

Reneses, B.

Rezai, A.R.

Rezak, M.

Ribot, R.

Rios-Lago, M.

Rivera, P.

Rizer, K.

Roberts, D.W.

Rodriguez, W.

Rolston, J.D.

Romero, C.

Rosenow, J.M.

Ross, E.K.

Rossi, F.

Rossi, J.

Rowland, N.C.

Sachs, A.J.

Sacino, $M$.

Salas, S.J.

Salazar, O.

Sammartino, F.

San Martin, E.S.

Sanchez-Casarrubios, S.

Sani, S.B.

Sankhla, T.

Santos, A.
$34,41,69$

13,50

71

$10,40,46$

16

55,56

17

16

22,42

14

$10,36,40$

65

40, 42

$31,40,42$

14

32

59

24

54

5

10,19

37

30

16

16

50

7

59

21,56

27,29

$12,37,48,68$

55

58

50

4

19

22

1,17

16

4

35

16

11,39

54

27, 29
Sapiro, G. 11

Sather, M.D. 18

Scharre, D.W.

10

Schevon, C.

63

Schiess, M.

53

Schlein, A.

26

Schroeder, K. 48

Schulder, M.

$1,16,17$

Schüpbach, M.

58

Schuurman, P.

57

Schwalb, J.M.

6

Schwartz, H.

5

Sederberg, P.

10,19

Segar, D.J.

53,54

Seidler, R.D.

51

Seijo, F.

35

Serrano, E.

30

Servello, D.

35

Settell, M.

55

Shaikhouni, A.

19

Sharan, A.D.

$21,23,49$

Sharim, J.

41

Sharma, G.

19

Shenai, M.B.

54

Sheth, S.A.

4, 25, 63

Shetter, A.G.

60

Shin, D.

36

Shiu, T.

28

Shouval, H.

62

Shu, H.

8

Silverman, A. $\quad 52$

Silverman, D. 63

Slavin, K.V. 12

Slevin, J. $\quad 55$

Smith, E.

25,63

Smith, H.C.

36

Smith, J.H. Jr.

56

Sobstyl, M.R.

$41,45,58$

Soltanian-Zadeh, H. 6

Son, B.-C.

$27,66,66,67,67$

Spencer, D.D.

49

Spiegelmann, R.

35

Starr, P.A.

$1,13,56$

Steel, D.

38

Steinke, G.K.

26, 57

Stern, M.A. 
Strange, B. $\quad 5$

Sukul, V.V. $\quad 59$

Sultzer, D. 5

Sundararajan, S. $\quad 15$

Swann, N. 13

Taira, T. 50

Tao, J. 22

Tat, D. 48

Teixeira, M.J. $\quad 9,59,68$

Thompson, J.

Tierney, T.S.

Timmermann, L. $\quad 35$

Timoney, N.S. $\quad 56$

Tomycz, N.D. $\quad 70,71$

Tonelli, M.P. 43

Tran, D.K.T. $\quad 17,26$

Tung, J. 28

Ungar, L. $\quad 35,50$

Vadera, S. $\quad 17,26$

Vagkopoulos, K. 8

Vale, F.L. 24

Valerio, J.E. $\quad 69$

Van Dyck, N. $\quad 57$

van Horne, C.

Vasenina, $\mathrm{V}$.

Velez-Ruiz, $\mathrm{N}$.

Verin, $M$.

Vivas, A.C.

Volkmann, J.

Wagstaff, W.

Walker, H.

Wang, D.D.

Wang, W.

42

30

35

24

57

34

54

1

9
Warnke, P.C.

22,42

Waseem, H.

24

Weber, P.B.

7

Weichart, E. $\quad 10$

Welker, K.M. 24

Welleford, A. $\quad 55$

Whiting, D.M. 45

Whone, A. 35

Wieckowski, B. 30

Williams, Z. 62

Willie, J.T.

$28,32,33$

Witcher, M.R. 33

Wolf, A.L. $\quad 69$

Woodroffe, R. 32

Wright, M. 51

$\mathrm{Wu}, \mathrm{C} . \quad 21,23,49$

$\mathrm{Xu}, \mathrm{C}$.

70,71

Yamamoto, E. 39

Yeagle, E. 23, 65

Yin, D.

31,43

Youn, Y.

10

$\mathrm{Yu}, \mathrm{H}$.

59

Zabek, M.

$41,45,58$

$37,55,56$

Zaczynski, A.

45

Zadikoff, C.

37

Zaghloul, K.A. 61

Zanaty, M. 32

Zavala, B. 61

Zhang, J. $\quad 10,52$

Zibly, Z. $\quad 50$

Zillgitt, A. 6

Zimmerman, R.S. 7 